\title{
LANCI DI GALLEGGIANTI \\ PER LO STUDIO DELLE CORRENTI SUPERFICIALI NEI BACINI LIGURE E TIRRENICO $\left(^{*}\right)$
}

\author{
Norberto Della Croce
}

\section{I. -- INTRODL ZIONE.}

1. - Scopo della presente ricerca, di cui venne data notizia in una nola preliminare ( $\left.{ }^{1}\right), \dot{e}$ di estendere ulteriormente le nostre conoscenze sul regime idrodinamico superficiale dei mari Ligure e Tirreno, e delimitare possihilmente le masse d'acqua, a caratteristiche di trasporto poco definite per direzione e nel tempo, che vi partecipano, per prepararne l'indagine diretta con osservazioni fisico-chimiche.

Particolare interesse hiologrico è dato dalle correlazioni che possono sussistere tra le caratteristiche di questo moto ed il trasporto e la distribuzione del plancton, unitamente alla migrazione di alcune forme ittiche, indicando il moto stesso lo spostamento dello strato più superficiale delle acque, e sul quale l'effetto di propulsione del vento è margiomente sensibile.

1. 2. - L'ampio esame hibliografico eseguito da $O$. Marinelli e Platania $(-)$, relativo alle prime indagini sulle caratteristiche grenerali della circolazione superficiale mediterranea e della probahile esistenza di questa nei mari Ligure e Tirreno, favorisce, nella nostra ricerca, un esame dei risultati ottenuti e delle osservazioni raccolte a partire dall'inizio del secolo.

1. 2. 1. - Il periodico "La Marina Mercantile Italiana " ( $\left.{ }^{3}\right)$ segnala, in uno scritto di autore ignoto, l'avvenuta misurazione della velocità della corrente litorale nel Golfo Ligure per mezzo delle prove di macchina del "Principe di Udine ".

O. Marinelli ( 1 ), esteso l'esame ad altre prove di velocita di numerosi piroscaf, ritiene dubbia l'esistenza di questa corrente e fa riferimento a prohahili moti di deriva (da vento), contrari al senso della litorale solo in estate per il prevalere di un regime isoluarico ad alte pressioni.

(") Comunicazione presentat:a al Convegno dell'Associarione Geofisica Italiana, ienuto a Roma il 17-18 giugno 1953. 
Dainelli, Marinelli e Stefanini (") eseguono sulle coste livornesi una serie di lanci di bottiglie accoppiate tipo Hautreux, e concludono - limitatamente al periodo della loro ricerca - non esservi nel Tirreno che movimenti a carattere di deriva da vento, e conseguentemente variabili nelle diverse stagioni in dipendenza delle diverse forme isoluariche.

1. 2. 2. - In una nota preliminare su lanci di galleggianti accoppiati, effettuati nel Ligustico per conto del Comitato Talassografico Italiano, Marini $\left({ }^{6}\right)$ propende invece per un moto costante nel senso della litorale, indipendentemente dalle stagioni, il quale - nullo nella parte centrale del bacino - prende consistenza regolare ad una certa distanza dalla costa: mentre moti irregolari, variabili con le condizioni meteorologiche, si avrebluero in prossimità di essa.

I risultati di successivi lanci di galleggianti isolati, eseguiti nelle acque di Castiglioncello, inducono Baldasseroni e Stefanini ( ${ }^{7}$ ) a ritenere non esservi nel Tirreno correnti continue, ma unicamente moti di deriva con direzione e velocità varianti con la direzione e la forza del vento. La prevalenza di un regime isolorico a carattere depressionario, gravitante tra il Mar Ligure ed il Tirreno, provocherebhe, "nel semestre invernale", moti da deriva nel senso della litorale; nei mesi estivi, non prevalendo decisamente un tipo isoluarico, i movimenti risulterelblero meno veloci e meno uniformi.

1. 2. 3. - Marini $\left({ }^{s}\right)$, condotte a termine le esperienze precedentemente iniziate, oltre a confermare le preliminari deduzioni ritenute prive di valore dimostrativo dai predetti Autori (9), indica la posibile esistenza di altri rami di corrente, quali completamenti occasionali della normale circolazione. Presumilhilmente all'altezza delle isole Hyéres, uno di essi si diparte dalla litorale, ragugiunge le acque nordoccidentali corse e tende per $\mathrm{NE}$ verso i lidi della Versilia, originando il circuito ciclonico chiuso del Mar Ligure. Un ulteriore frazionamento di questo ramo, in prossimita della costa corsa raygiunge quella meridionale sarda sul versante occidentale. Nelle acque tirreniche il predetto circuito ciclonico, diretto verso i lidi toscani, origina una corrente da $\mathbf{N}$ a $\mathrm{S}$ la quale, tra Ostia ed il Golfo di Napoli, tende verso $W$ per scindersi in un ramo ascendente a completamento di un eventuale circuito anticiclonico dell'Alto Tirreno, e in uno discendente che rientra in quello ciclonico del Medio e Basso Tirreno. 
1. 2. 4. - Ricerche idrografiche di Trotti $\left({ }^{10}\right)$, sulle acque costiere ligustiche, comprendono una serie di lanci di galleggianti accoppiati al largo del Promontorio di Portofino, e dei quali attualmente non sono stati pubblicati $i$ risultati.

1. 3. - Meno numerose indagini furono eseguite al contrario nel Medio e Basso Tirreno. Interessano questa area di mare alcuni lanci degli esperimenti condotti dalla spedizione oceanografica danese. Platania $~^{11}$, che ne ha studiato $i$ risultati, tende a riconfermare in via generale le conclusioni di $O$. Marinelli : un moto ciclonico o anticiclonico delle acque sarebbe in stretta dipendenza con le condizioni del campo barico, tuttavia il ramo che corre parallelo alla costa settentrionale sicula può considerarsi - secondo l'A. - come vera corrente, a direzione costante e con velocita decrescente per forti venti contrari.

1. 3. 1. - G. Marinelli (12), a conclusione di successive esperienze, ammetterebhe l'esistenza di una controcorrente molto vicina alla costa ( già parzialmente notata dal Marini con diversa interpretazione), dal Canale di Piombino alle isole Eolie, ove piega ad Occidente per l'influenza di acque del hacino mediterraneo orientale provenienti dallo Stretto di Messina. L'A. inoltre, richiamandosi a precedenti indagini ("), ritiene che il circuito ciclonico del Basso Tirreno si spezzi in prossimita del Golfo di Napoli, e non a $S$ della Sardegna come indicato dal Nielsen.

1. 4. - Alle precedenti ricerche intese a determinare per via indiretta la circolazione superficiale dei bacini ligure e tirrenico, si uniscono quelle idrografiche della spedizione danese del Thor.

Secondo Nielsen $\left({ }^{13}\right)$ e Schott $\left({ }^{14}\right)$ all'altezza della costa tunisina, un ramo che si diparte dalla corrente atlantica, raggiunge le coste settentrionali sicule, scorre per NW parallelamente a quelle italiche, tende sul Golfo Ligure e prosegue per $\mathrm{SW}$ lungo le coste franco-ispane. La litorale, inoltre, all'altezza del Golfo di Napoli, origina un ramo per $\mathrm{W}$ e SW in direzione delle coste meridionali sarde (circuito del Nielsen l.

\section{II. - METOdo DI INDAgINE.}

2. 1. - Come si vede le nozioni acquisite al riguardo delle correnti superficiali dei bacini ligure e tirrenico non sono molto complete: ed il Centro Talassografico Tirreno si è proposto di riprendere le indagini anche con nuovi lanci di galleggianti, simultanei a quelli 
progettati, per analoga iniziativa francese, nell'atligua estensione marina, ad Occidente della Corsica e della Sardegna.

1 presenti risultati furono ottenuti con lanci di gruppi di galleggianti isolati, nei Mari Ligure e Tirreno nelle quattro stagioni del 1952, in mare aperto a notevole distanza dalla costa, pressoché simultaneamente, da cinque stazioni prestabilite: poiché tutte le precedenti esperienze avevano avuto luogo di massima in immediata vicinanza dei litorali, si ritenne opportuno che queste cinque stazioni fossero scelte al largo, per evitare l'influenza degrli irregrolari e variabili moti costieri, e per osservare il comportamento delle acque di altura rispetto alla litorale ed alle condizioni meteorologiche; i cinque lanci di ciascuna stazione vennero eseguiti quasi simultaneamente, per permettere un esame di insieme sul comportamento delle diverse aree prese in esame ed una più attendibile deduzione del percorso compiuto dalle bottiglie rinvenute, anche in dipendenza delle situazioni hariche influenzanti le condizioni dei due mari.

2. 2. - Le posizioni greografiche delle cinque stazioni di lancio vennero stabilite, unitamente al proposito di effettuare esperienze al largo delle coste, in maniera da prendere in esame zone che già dai risultati delle precedenti indagini apparivano offrire maggiore interesse. Si ritenne cosi particolarmente utile effettuare lanci nella parte centrale del Mar Ligure, nelle acque dell'arcipelago toscano, ed al limite di separazione tra l'Alto ed il Medio Tirreno, nonché tra le roste partenopee e sarde, e tra queste e le sicule; le stazioni di lancio vennero pertanto stabilite nei punti intermedi di cinque rotte che attraversano i Mari Ligure e Tirreno in senso quasi normale alla costa italiana, ricorrendo all'appogrgio dei servizi marittimi della Societa di Navigazione "Tirrenia ", che si prestò gentilmente ad incaricare del lancio i comandanti delle unità dipendenti.

Le rotte prescelte furono Genova-Porto Torres, Livorno-Bastia, Civitavechia-Olhia, Napoli-Cagliari e Cagliari-Palermo. Complessivamente vennero gettati a mare 1100 galleggianti. In ogni stazione si lanciarono gruppi di cinquanta bottiglie nel lancio invernale, estivo ed autunnale e di settanta nel lancio primaverile.

Nel 1952 i quattro lanci eseguiti in ciascuno di detti cinque punti furono effettuati, rispettivamente: tra il 28 ed il 30 gennaio; tra il 23 ed il 26 marzo; tra il 29 gingno ed il 2 luglio (*); e tra il 2 ed il 6 novembre.

(*) Causa disguido Cerroviario, il gruppo sulla rotta Napoli-Cagliari fu forzatamente lanciato solo il 15 luglio 1952. 


\section{III. - Esame statistico dei ristltati.}

3. 1. - Galleggianti lanciati in acque d'altura, con notevoli possibilità di dispersione, dànno valori percentuali di ricupero molto vicini a quelli delle precedenti indagini eseguite in prossimita delle coste $\left({ }^{*}\right)$.

Tarbl.ta I - Ricuperi e valori percentuali.

\begin{tabular}{|c|c|c|c|c|c|}
\hline $\begin{array}{l}\text { Stazioni } \\
\text { sulle rotte }\end{array}$ & $\begin{array}{l}\text { I lancio } \\
28.1 .1952 \\
30 . I .1952\end{array}$ & $\begin{array}{c}\text { II lancio } \\
23.111 .1952 \\
26.111 .1952\end{array}$ & $\begin{array}{l}\text { III lancio } \\
29 . \text { VI.1952 } \\
2 . \text { VII.1952 }\end{array}$ & $\begin{array}{c}\text { IV lancio } \\
2 . X I .1952 \\
6 . X 1.1952\end{array}$ & Totale \\
\hline Cangliari- & 22 & 21 & 4 & 20 & 67 \\
\hline Palermo & $44 \%$ & $30 \%$ & $8 \%$ & $40 \%$ & $30 \%$ \\
\hline Napoli- & 23 & 21 & 2 & 13 & 59 \\
\hline Cagliari & $46 \%$ & $30 \%$ & $4 \%$ & $26 \%$ & $26 \%$ \\
\hline Civitaverchia- & 19 & 33 & 4 & 13 & 69 \\
\hline Olbia & $38 \%$ & $47 \%$ & $8 \%$ & $26 \%$ & $31 \%$ \\
\hline livorno- & 29 & 36 & 13 & 11 & 89 \\
\hline Bastia & $58 \%$ & $51 \%$ & $26 \%$ & $22 \%$ & $40 \%$ \\
\hline Genova- & 12 & 25 & 27 & 5 & 69 \\
\hline Porto Torres & $24 \%$ & $35 \%$ & $54 \%$ & $10 \%$ & $31 \%$ \\
\hline \multirow{2}{*}{ Tolale } & 105 & 136 & 50 & 62 & 353 \\
\hline & $42 \%$ & $38 \%$ & $20 \%$ & $24 \%$ & $32 \%$ \\
\hline
\end{tabular}

(4) Nel Mar Ligure Marini ricupero rispettivamente il 17\% (1913), il 32\% (1911) ed il 36\% (1920) dei galleggianti lamciati. Per l'Alto Tirreno fa ecrezione l'elevata percentuale del 50\% ottenuta da Dainelli, Marinelli e Stefanini (1909.10), mentre Stefanini e Baldasseroni (1919.20) conseguirono il 29\%. Nel Medio e Basso Tirreno G. Marinelli ne rinvenne, da esperienze effettuate saltuariamente tra il 1924 ed il 1928, il 33\%. Ricerche condotte dalla spedizione oceanografica damese in questa area di mare (agosto 1912-febbraio 1913) portarono a ritrovamenti pari al $10 \%$. 
L'estensione superficiale del Mar Tirreno tra la penisola italiana ad Oriente ed i lidi corsi e sardi ad Occidente, la sua ridotta superficie a Nord in prossimità dell'arcipelago toscano, e la maggior superficie a Sud, spiegherebhero la più alta percentuale totale di ritrovamenti conseguita per i lanci effettuati sulle rotte Livorno-Bastia (40\%) e la minor percentuale sulla rotta Napoli-Cagliari (26 $/ \mathrm{c})$; aggirandosi sul 30 e $31 \%$ quelle relative alle altre stazioni.

Le hottiglie complessivamente ricuperate ammontano a 353 , pari al $32 \%$. I lanci che portarono al maggior numero di ritrovamenti furono quelli del grennaio $(42 \%)$ e del marzo $(38 \%)$.

I valori percentuali ottenuti dai ricuperi dei lanci estivi ed autunnali inducono a ritenere che nel Mar Ligure ed Alto Tirreno da un lato, e nel Medio e Basso Tirreno dall'altro. si vengono a creare in differenti stagioni condizioni ad opposto comportamento.

3. 2. - La frequenza di distribuzione dei galleggianti nei diversi tratti della costa mediterranea permette di osservare il possibile sussistere di relazioni tra le zone di lancio e quelle di ricupero.

Nella tavola seguente si è creduto opportuno riunire i litorali ove avvennero $i$ ritrovamenti in due gruppi distinti, corrispondenti ad una eventuale circolazione ligure-tirrenica da un lato, e ad una eventuale circolazione balearica dall'altro. Il primo va dalle coste occidentali sicule a quelle orientali sarde con "vertice " settentrionale in Liguria: il secondo si diparte dai litorali meridionali francesi e, raggiunti quelli iberici, termina sul versante occidentale della Corsica e della Sardegna: dette coste sono elencate nel senso della litorale e della normale circolazione mediterranea, onde rendere più agevole l'interpretazione dei risultati.

I ritrovamenti delle bottiglie lanciate dalle stazioni poste sulle rotte Cagliari-Palermo e Napoli-Cagliari sembrano indicare l'esisten. za di un circuito interessante il bacino tirrenico, con esclusione del Ligustico, essendo i ricuperi più settentrionali limitati alla costa toscana ed orientale corsa. Tuttavia, non sembra esservi al limite di separazione dei due mari - tra Capo Corso e l'Isola d'Elba - sharramento alcuno per le bottiglie lanciate sulle rotte Civitavecchia-Olbia e Livorno-Bastia, le quali, raggiunto il Mar Ligure, continuano il loro percorso nel circuito halearico. I risultati conseguiti dai lanci effettuati sulla rotta Genova-Porto Torres, oltre a confermare l'esistenza di un moto nella parte centrale del bacino ligustico, indicano 
come detto moto si avvicini alle coste in prossimita del Var, da dove è ancora possibile seguirne il decorso solo frammentariamente lungo le coste iberiche.

Tabelia II - Frequenza di distribuzione dei galleggianti rinvenuti sulle coste medilerranee.

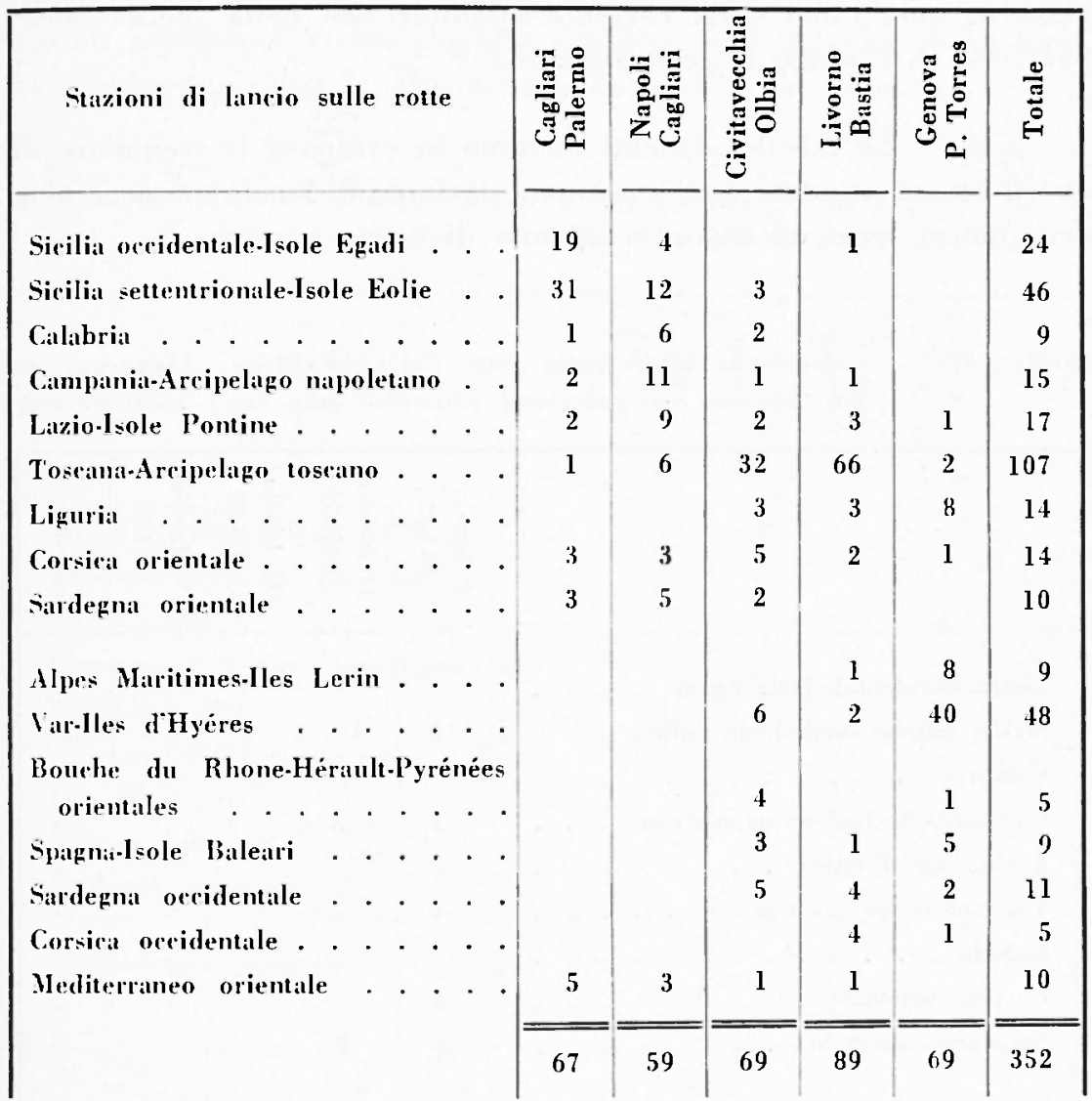

Nella voce "Mediterraneo orientale " sono inclusi anche i ricuperi avvenuti sulle coste meridionali ed orientali sicule, e meridionali ed occidentali calabre.

Tali condizioni inducono quindi a ritenere che le bottiglie dei lanci effettuati sulle prime due stazioni considerate, giunte all'altezza dell'arcipelago toscano, siano state trascinate verso $S$ lungo le coste orientali corse e sarde, per la formazione del circuito ciclonico chiuso del Mar Ligure, o per la prevalenza occasionale di venti settentrionali.

Scarsi sono i ritrovamenti a $S$ delle stazioni di lancio; aggiunge- 
remo che la marrior parte di essi si eblue in occasione della esperienza estiva. la quale diede la più hassa percentuale di ricupero.

I galleggrianti lanciati dalla stazione situata sulla rotta NapoliCagliari e ricuperati sulle coste settentrionali sicule possono attribuire il loro moto verso $S$ a trascinamento da vento, oppure a trasporto, da parte del circuito ciclonico del Basso Tirreno, sino a venire ripresi in quel ramo della corrente atlantica clue dalla Sicilia corre poi verso $\mathrm{NW}$ lungo le coste italiane.

3. 3. - Le tabelle sequenti mettono in evidenza le frequenze di distribuzione ottenute per le cinque stazioni di lancio in occasione dei quattro lanci effettuati in ognuna di esse.

Tabfla III - Stazione di lancio sulla rolta Cagliari-Palermo: Frequenza di distribuzione dei gallegiamti rincenuti sulle coste mediterranee.

\begin{tabular}{|c|c|c|c|c|c|c|c|}
\hline & & & - & 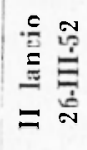 & 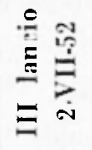 & 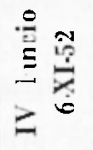 & 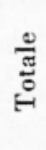 \\
\hline Sicilia occidentale-Isole Egadi . & . . & . . & & 1 & 1 & 17 & 19 \\
\hline Sicilia settentrionale-Isole Eolie. & . . & . . & 14 & 15 & 2 & & 31 \\
\hline . . . . . . . . & . . & . . & & 1 & & & 1 \\
\hline Campania-Arcipelago napoletano & . & . . & 1 & 1 & & & 2 \\
\hline Lazio-Isole Pontine . . . . . & . . & . . & 2 & & & & 2 \\
\hline Toscanil-Arcipelago toscano . . & . & . . & 1 & & & & 1 \\
\hline Liguria . . . . . . . . . . & . & . $\cdot$ & & & & & \\
\hline Corsical orientale . . . . . . . . & . . & . & 3 & & & & 3 \\
\hline Sardegna orientale . . . . . & $\cdot$ & - . & 1 & 2 & & & 3 \\
\hline Mediterraneo orientale. . . & $\cdot \cdot$ & . & & 1 & 1 & 3 & 5 \\
\hline & & & 22 & 21 & 4 & 20 & 67 \\
\hline
\end{tabular}

3. 3. 1. - I risultati del lancio invernale indicano un moto ciclonico interessante le acque tirreniche con esclusione delle liqustiche; tale movimento, insieme con la successiva modificazione indicata dal lancio primaverile, costituireble il circuito ciclonico del Basso Tir- 
reno; nessuna conferma della sua esistenza ed ampiezza viene suggerita da ricuperi della esperienza estiva, la quale - anche in considerazione degli scarsi ritrovamenti - induce a ritenere che le condizioni determinanti la precedente fisionomia idrodinamica ahhiano su. bito modificazioni notevoli; i ritrovamenti del quarto lancio, esclusivamente in direzione $\mathrm{SE}$, trovano spiegazione nell'intervento di particolari condizioni meteorologiche e purtroppo non permettono aleuna conclusione circa la circolazione dominante in autunno.

Tabela. IV - Stazione di lancio sulla rota Sapoli-Cagliari: Frequenza di distribuzione dei galleggianti rinvenuti sulle coste mediterranee.

\begin{tabular}{|c|c|c|c|c|c|c|}
\hline & & 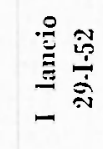 & 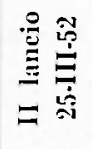 & 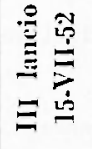 & 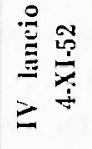 & 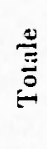 \\
\hline Sicilia orcidentale-Isole Egardi . & . & I & 3 & & & 4 \\
\hline Sicilia seltentrionale-Isole Eolie. & . $\cdot$ & 8 & 2 & 1 & 1 & 12 \\
\hline Callabrial . . . . . . . . . . & . & $\underline{2}$ & 1 & & 3 & 6 \\
\hline Campania-Arripelago napoletano & . & 6 & & & 5 & 11 \\
\hline Lazio-Isole Pontine . . . . . & . & 1 & 4 & 1 & 3 & 9 \\
\hline T'osémil-Arcipelago toscano . . & $\cdot$ & 3 & 3 & & & 6 \\
\hline Liguria . . . . . . . . . & · $\cdot$ & & & & & \\
\hline Corsica orientale . . . . . . & $\cdot$ & & 3 & & & 3 \\
\hline Sardegna orientale . . . . . & . & & $t$ & & 1 & 5 \\
\hline Mediterraneo orientale . . . & $\cdot \cdot$ & 2 & 1 & & & 3 \\
\hline & & 23 & 21 & 2 & 13 & 59 \\
\hline
\end{tabular}

3. 3. 2. - L'esistenza del moto a carattere ciclonico, segnalato dai risultati dei lanci effettuati sulla rotta Cagliari-Palermo, sembra trovare conferma in quelli eseguiti sulla rotta Napoli-Cagliari. L'esame più accurato delle schede ritrovate mostrerà tuttavia come detto moto, a caratteristiche cicloniche ben definite per il lancio d'inverno, risulti interessare con minor completezza, unitamente al circuito del Nielsen, larea tirrenica in occasione dei lanci primaverile ed autunnale. 
Tapella V - Stazione di lancio sulla rotha Civitavecchia-Olbia: Frequenza di distribuzione dei galleggianti rinvenuti sulle coste mediteranee.

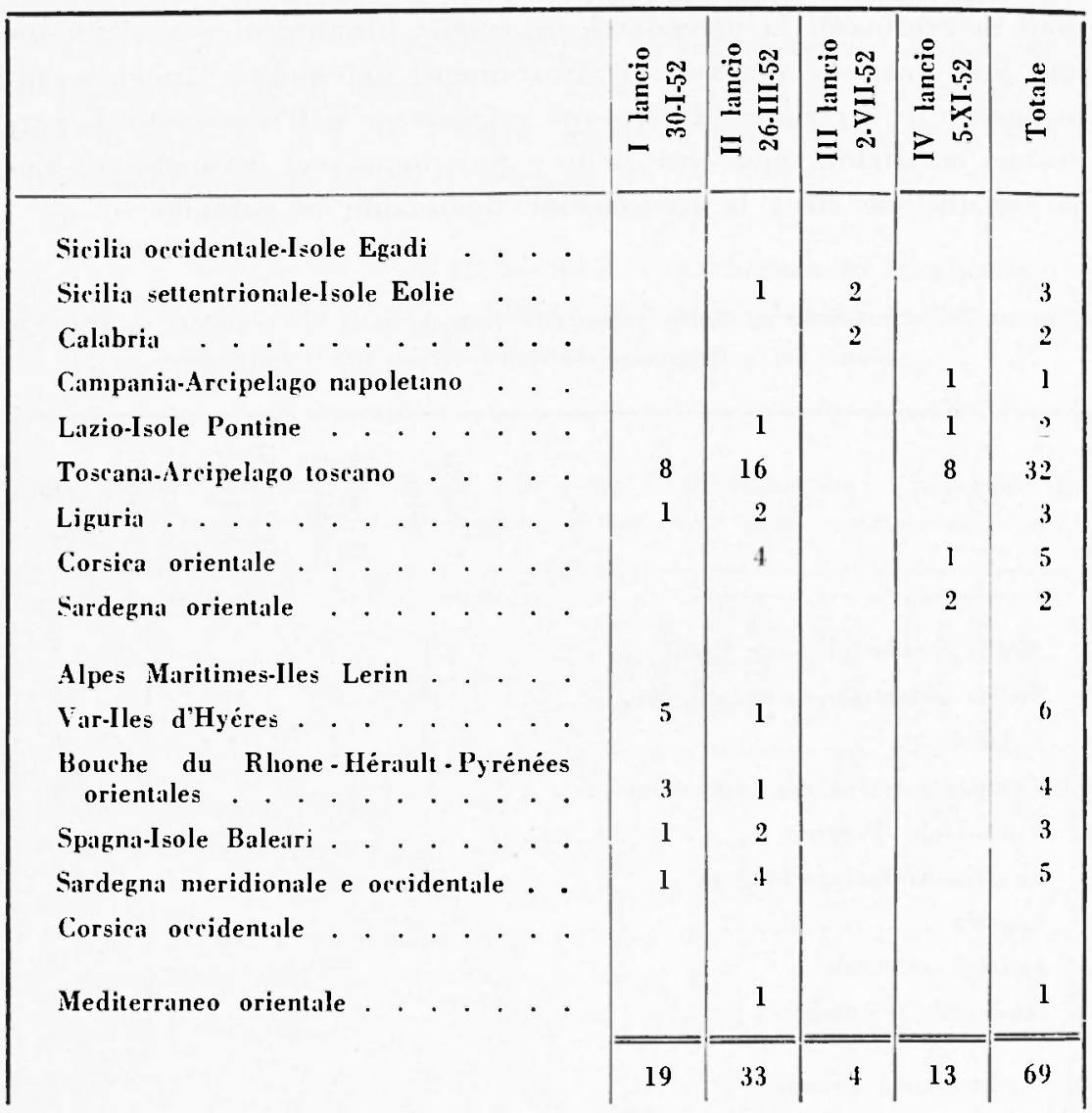

3. 3. 3. - Il lancio invernale e quello primaverile comprovano l'esistenza del circuito ciclonico identificantesi con la radente mediterranea della quale $\mathbf{i}$ galleggianti ricuperati mostrano aver seguito il percorso sino all'altezza delle coste iberiche, accennando inoltre, all'esistenza di un circuito balearico esaurentesi nei ritrovamenti effettuati sulle coste occidentali sarde. Altre bottiglie, ricuperate in Sicilia e nel Mediterraneo orientale, attestano l'ulteriore propagazione verso $\mathrm{E}$ del ramo della corrente atlantica.

L'esperienza estiva ottiene, al contrario, risultati diversi. 
TapFLLA VI - Stazione di lancio sulla rolta Livorno-Battia: Frequenza di distribuzione dei galleggianti rinvenuti sulle coste mediterranee.

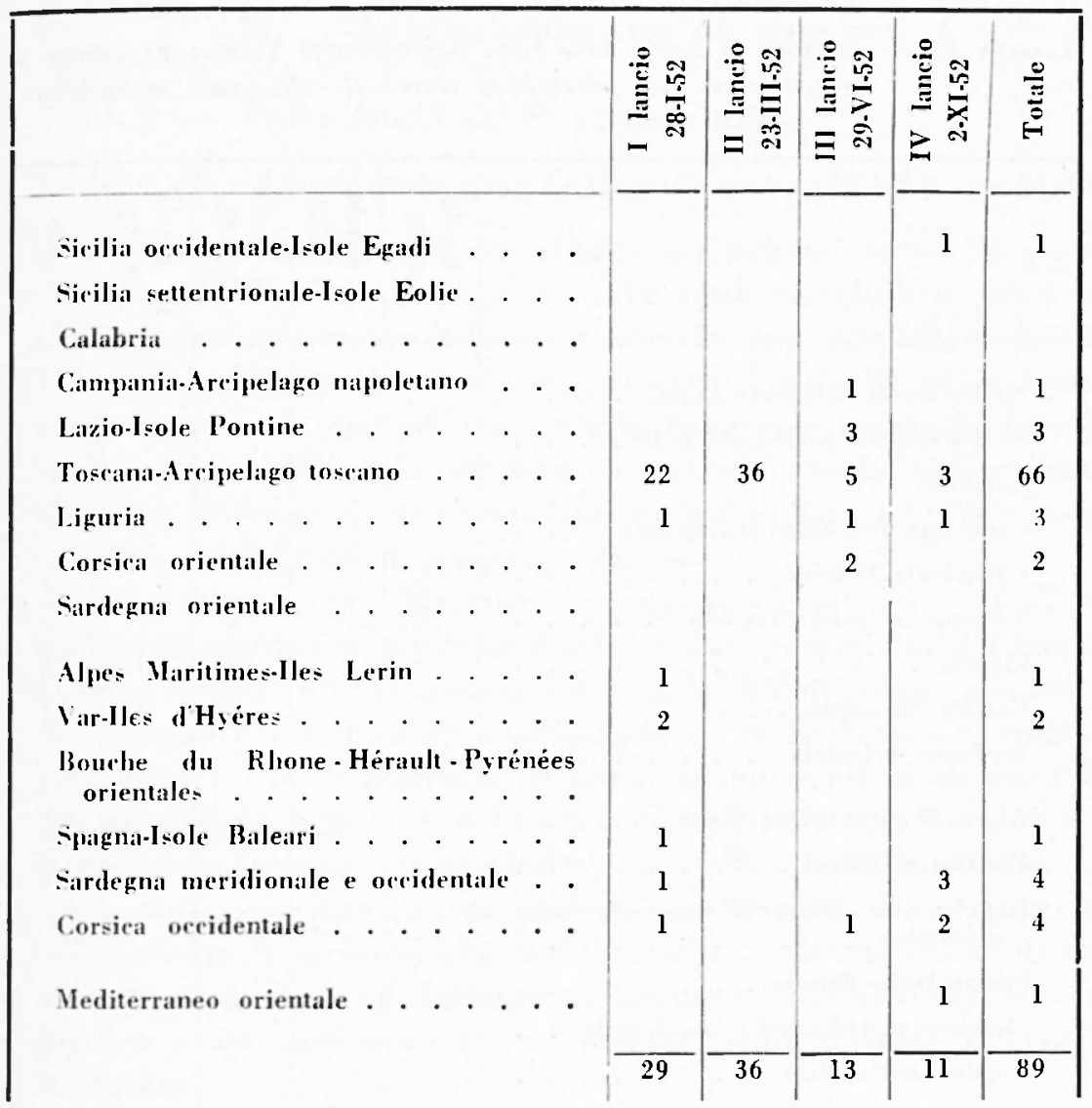

3. 3. 4. - Il lancio invernale conferma la continuazione della radente mediterranea nel circuito balearico. Quello primaverile, con ricuperi su esiguo tratto del litorale toscano, in breve volgere di tempo, e più a Nord del punto di lancio, ha purtroppo scarso valore per il problema, dovendosi attribuire i risultati (come per il lancio d'autunno sulla rotta Cagliari-Palermo) a particolari condizioni meteorologiche della regione. Un moto contrario alla radente sembra risultare dal lancio estivo, mentre i risultati di quello autunnale, con ritrovamenti sui versanti occidentali della Corsica, della Sardegna e sulle 
coste meridionali sicule, non si presta a deduzioni di sicura interpretazione.

Tabella VII - Stazione di lancio sulla rolta Genova-Porto Torres: Frequenza di distribusione dei galleggianti rintenuti sulle coste mediterranee.

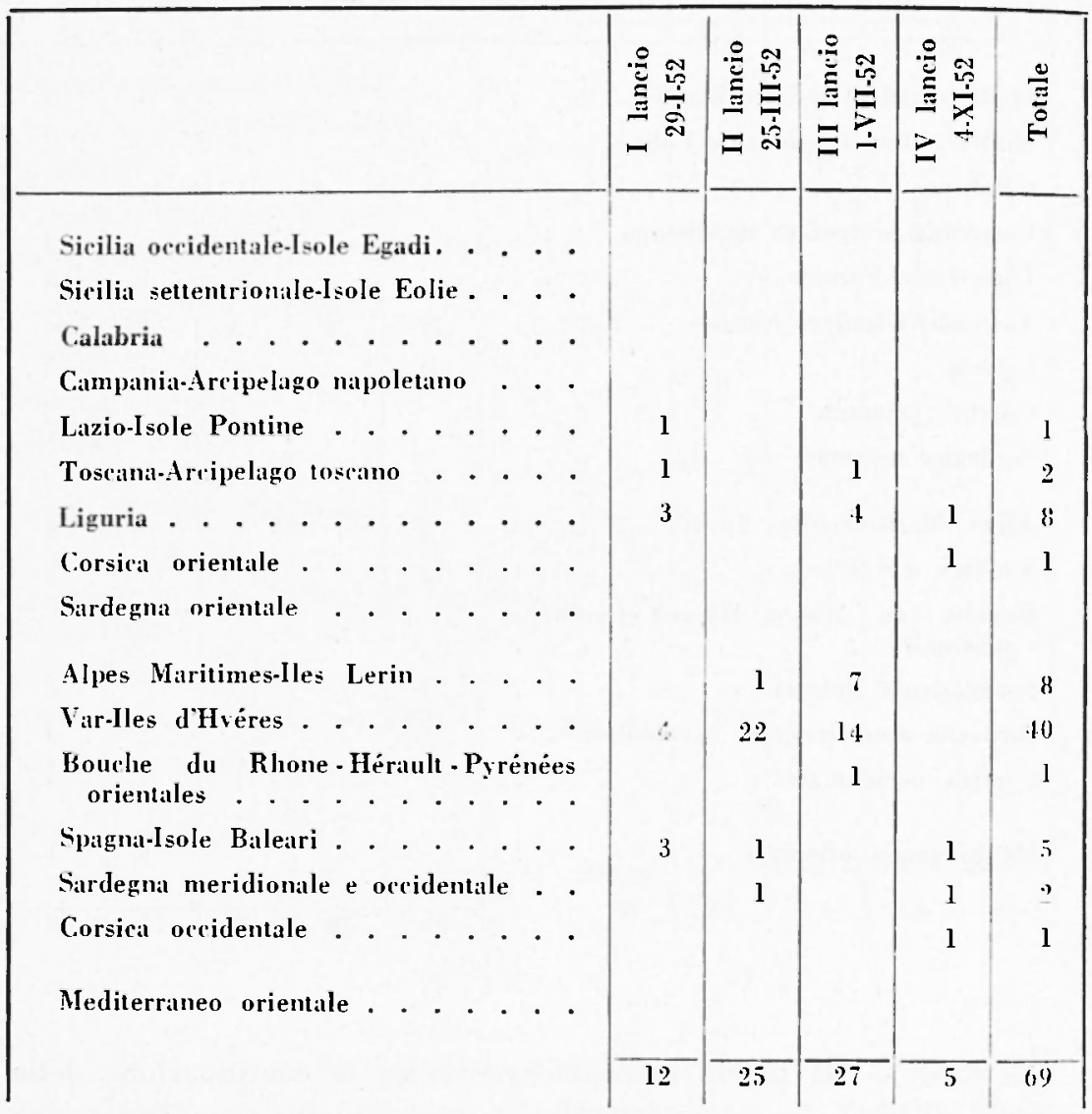

3. 3. 5. - Ricuperi sulle coste francesi ed ispaniche di grallewgianti appartenenti ai primi tre lanci, confermano la presenza di una corrente radente da Nord-Est a Sud-Ovest lungo i predetti litorali. L'esperienza d'autunno, con ritrovamenti sulle coste occidentali corse e sarde, mostra l'esistenza di condizioni mollo simili a quelle rilevale 
nel lancio autunnale eseguito due giomi prima sulla rotta LivornoBastia.

\section{IV. - Esane cartografico dei risultati.}

4. 1. - Primo lancio (28-30 gennaio 1952).

4. 1. 1. - Lancio sulla rotta Cagliari-Palermo (30-1-52, ore 01.00 ।.

L'esame cartografico e cronologico dei ricuperi indica di massima una diffusione dei galleggianti sulle coste tirreniche in senso ciclonico: particolarmente indicativi i ritrovamenti delle schede n. 112. 113, 106, 127 e 126: le ultime due, rinvenute in luglio ed in agosto rispettivamente a due ed a un miglio dalle coste orientali corse e sarde, esaurireblero tale diffusione iniziatasi su quelle settentrionali sicule in febluraio (112), e segnalata sui litorali laziali (113) e toscani (106) dai ricuperi di maggio e giugno.

Le schede n. 128 e 125 , ritrovate (maggio e giugno) a 2 e 4 miglia dalle coste nord-orientali della Sicilia a oltre cento giomi dal lancio, quando in maggio si ricuperava tra le isole dell'arcipelago pontino il gallegrgiante 113, inducono ad ammettere - anche in base a risultati precedenti $\left(^{*}\right)$ - l'esistenza nelle acque calabro-sicule di un moto da NW per SE. Lo spiargriamento di numerose bottiglie nel Golfo di Castellamare (Trapani), principalmente a $22-23$ giomi dal lancio, sareble stato provocato da una corrente secondaria a vortice generata dalla principale in prossimita di Capo Gallo, o da una azione di trascinamento da vento sui galleggianti. oppure da modificazione - sempre per causa anemonica - del percorso della corrente principale medesima.

11 Bollettino meteorologico dell'Aeronautica ( 15 in) rileva, nei giorni intercorsi tra lancio e ricupero, situazioni isobariche depressionarie sul Tirreno e sul Mar Ligure caratterizzate da venti occidentali e nord-occidentali sulla zona in questione, che le stazioni semaforiche di Capo Carbonara, Isola Favignana ed Isola d'Ustica registrano - specie nei primi giomi successivi al lancio stesso - come assai

(*) Gli esperimenti II e V di G. Yarinelli nel mese di maggio 1192.1 e 1925) a 22 e 26 miglia da Capo Palinuro danno esclusivamente ritrovamenti a $S$ del punto di lancio. 
forti, e con velocità medie gromaliere oscillanti tra le 40 e le 60 miglia all'ora $\left({ }^{*}\right)$.

4. 1. 2. - Lancio sulla rotla Napoli-Cagliari (29-1-52, ore 20.30).

I venti in precedenza segnalati, e comuni a tutto il Basso Tirreno, tendono a spostare $i$ gallegrgianti in direzione $\mathrm{SE}$ : $\mathrm{i}$ ritrovamenti delle schede n. 100 (20 miglia a NW dell'Isola Salina) e n. 80 (3 miglia a NW di Caronia Marina) permettono di apprezzare tale spostamento.

Considerate le diverse posizioni geografiche da cui nell'intervallo di cinque ore vennero eseguiti i lanci sulle due rotte Cagliari-Palemo e Napoli-Cagliari, i ritrovamenti tra le Isole Eolie, dopo $20-27$ wioni, dei gallegrgianti grettati a mare nei due punti (n. 112 per il primo e n. 100 per il secondof permettono di ritenere uniformi -- in quel momento $\rightarrow$ le condizioni idrodinamiche del Basso Tirreno.

I ricuperi, in aprile sulle coste partenopee ischede $n .65,88,58$. $70,87,57$ ) ed in magrio sull'Isola d'Elha (scheda n. 56), confermano, assieme a ritardati ritrovamenti $f^{\circ}$ । sulle coste losco-laziali del giumno-luglio (schede n. 61, 9:3, 92), la segnalata diffusione in senso ciclonico sulle coste tirreniche. A diflerenza del lancio precedente, non si hanno ritrovamenti sulle coste orientali corse e sarde, ma i ricuperi di luglio all'Isola d'Ustica ischede $n$. 64, 7.3), ai margini meridionali dell'area timenica, sembrano conferire alla sopra accennata diffusione i caratteri di un circuito ciclonico.

Bottiglie ritrovate successivamente, e griunte nelle acque sicule con le predette 64 e 73, indicano per il Basso Tirreno -.. a sei mesi dal lancio - modificate condizioni idrodinamiche. Le schede n. 82 e 5] ricuperate in luglio ed in agosto sulle coste occidentali e sud-occidentali sicule (dopo i reperti di Ustica), comproverebbero lesistenza di un moto da NW per SE, confermato anche dalla bottiglia n. 52, allontanatasi dalle acque tirreniche. Detto gallegriante, ripreso dal ramo della corrente allantica penetrante nel hacino mediterraneo orientale, veniva poi ricuperato sulle coste elleniche.

In tale periodo avrebhe svolgimento nel Basso Tirreno il circuito del Nielsen: indicative, al riguardo, le schede n. 9] e 97 che, griunte

(\%) La media giornaliera è stata ottenuta dai valori rilevati dagli strumenti in ogni stazione semaforica alle ore $08,1.1$ e 19.

$(*)$ Detti ricuperi potrebbero altresi indicare la contro corrente costiera segnalata da Marini e G. Marinelli. 


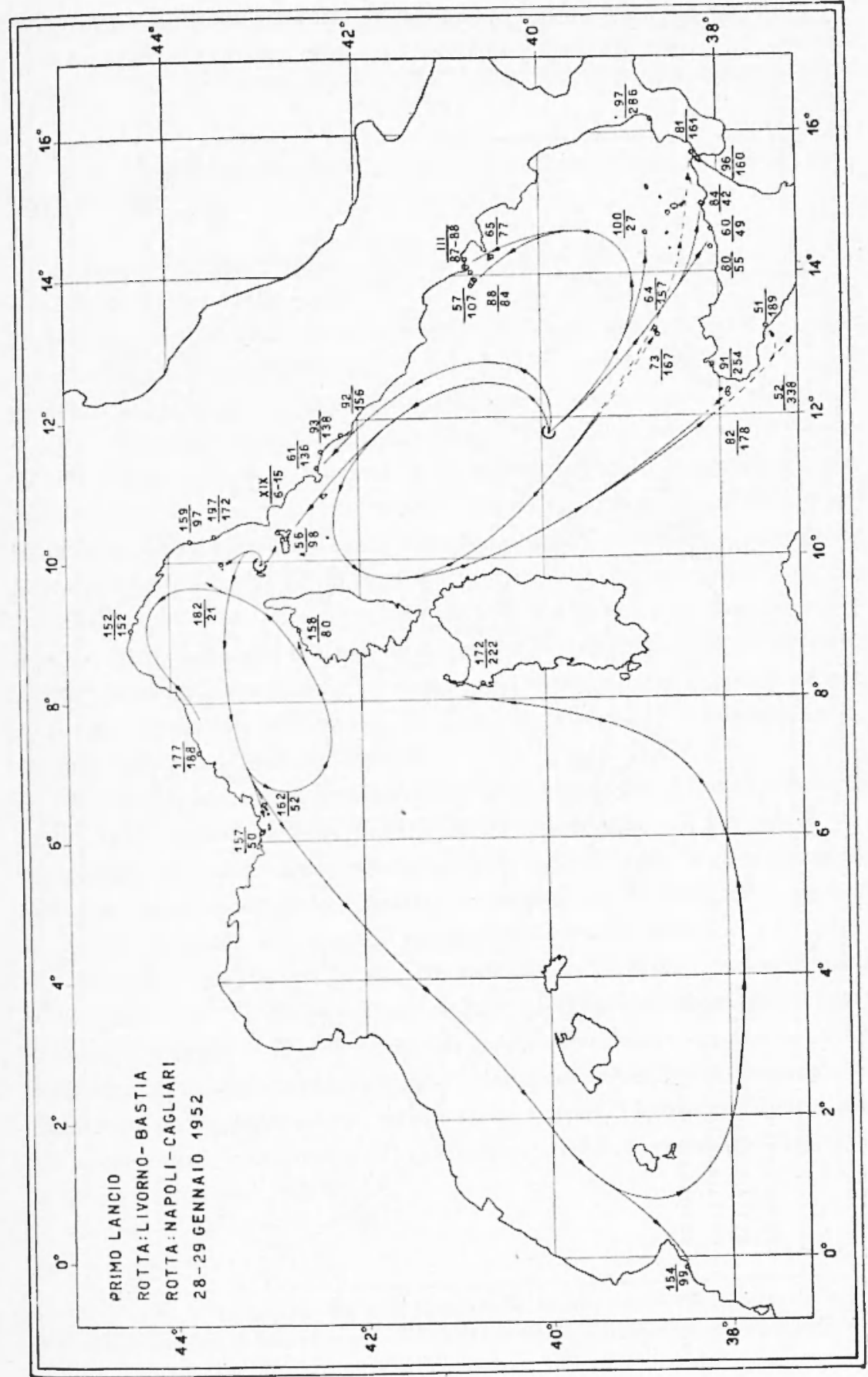




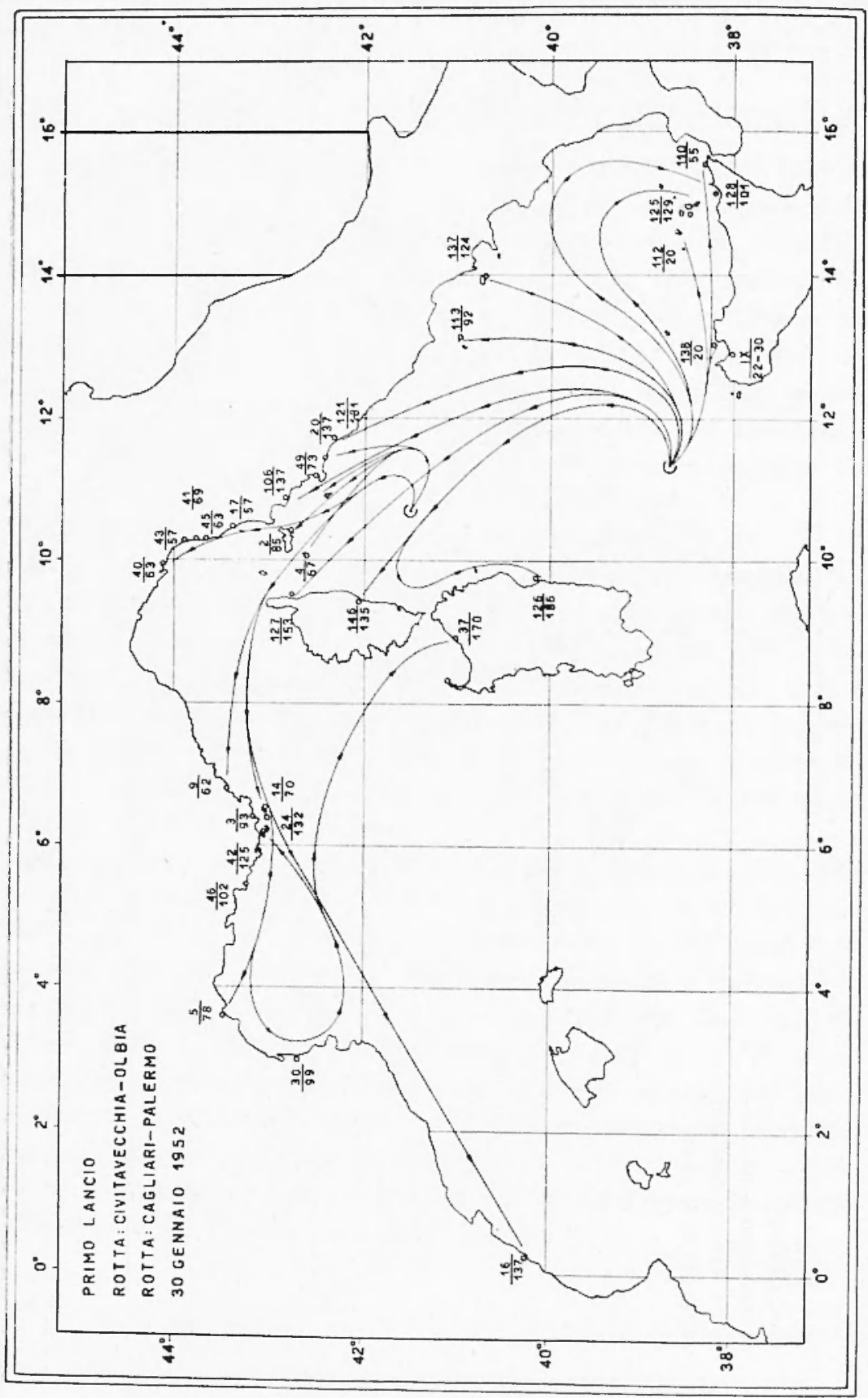


nelle acque siciliane ancora in luglio, seguito il predetto circuito, sarebhero poi spiagriate sulle coste nord-occidentali sicule in ottohre ischeda $n$. 91) ed occidentali calabre in novembre (scheda n. 97).

4. 1. 3. - Lancio sulla rotta Civitavecchia-Olbia (30-1-'52, ore (100.07).

I primi ricuperi sulle coste tosco-liguri da Rosignano Solvay (scheda n. 17) al Golfo della Spezia (scheda n. 40), tra il 27 marzo e l'8 aprile, ed altri (schede $n .9$ e 14) sulle coste sud-orientali francesi (pressoché contemporanei, tra il $1^{\circ}$ ed il 9 aprile), inducono a credere che $\mathrm{i}$ gallegrianti, separandosi in due rami a $\mathrm{S}$ o a $\mathrm{N}$ dell'Isola d'Elba, abbiano viagriato con modificata velocitì e direzione a seconda che siano venuti a trovarsi nella parte centrale o periferica del hacino ligustico. La corrente principale viene segnalata, infatti, quasi nello stesso periodo sulle coste toscane e liguri ad Oriente, su quelle francesi ad Occidente, ed in seguito sul versante iberico (scheda n. 16).

Dalle schede n. 42 e 24 , ritrovate nel Var ancora in giugno, risulterehbe nel Golfo del Leone, una corrente contraria alla principale, poiché penetratevi con le n. 5 e 30 (aprile-maggrio), avrebluero successivamente invertito, all'altezza di Capo de Creus, il cammino in direzione $\mathrm{NE}$ verso le Isole Hyéres.

Meno facile l'interpretazione del gallegriante n. 37 ricuperato in luglio sulle coste nord-occidentali della Sardegna: di probabile appartenenza al gruppo precedente (schede n. 5, 30, 42, 24), veniva spinto in un ramo secondario di completamento occasionale, con decorso da $N$ a $S$ parallelo al versante occidentale corso e sardo.

Altre hottiglie ( $n .4,2,49,20$ ) ricuperate in aprile (ad eccezione della scheda $n$. 20 ritrovata in giugno) nell'arcipelago e sulle coste toscane, e sempre a $\mathbb{N}$ del punto di lancio, avrebbero subito - a somiglianza di quanto avvenuto per i lanci in precedenza descritti l'influenza della depressione interessante $i$ mari Tirreno e Ligure. per cui spostandosi inizialmente in direzione $\mathrm{S}-\mathrm{SE}$, avehbero ritardato la loro risalita per N-NW (*).

(*) Non $i$ da escluders che tale ritardatania risalita verso $\mathrm{N}$ sia slata la causa dellinibito arcesso al Mar l.igure. ose nel fratlempo avrebbe preso consistenza il relativo circuito riclonico. 
4. 1. 4. - Lancio sulla rotta Livorno-Bustia (28-1-'52, ore 03.20).

1 primi ritrovamenti, sulle coste settentrionali dell'Isola d'Elba, denotano uno spostamento dei gallegrgianti per $\mathrm{SE}$, già rilevato chiaramente da quelli lanciati sulle rotte Napoli-Cagliari e Cagliari-Palermo. A dieciannove ammontano le lottiglie trascinate sulle coste ellane con primi ricuperi a sei giomi dal lancio. Il bollettino meteorologico dell'Aeronautica segnala lo spostamento di campi depressionari da NW per SE; i semafori di Capraia e Campo alle Serre (costa nord-occidentale elloana) registrano forti venti nord-occidentali specie a 5 giorni dal lancio stesso, con velocità media rispettiva di 60 e 50 mirlia all'ora. La corrente principale trova conferma nei ricuperi delle schede n. 182, 157, 162 e 154 ritrovate nelle acque di Gorgona in fehbraio, alle Isole Hyéres in marzo (dopo aver attraversato il hacino ligustico nella parte centrale e sulle coste meridionali ilseriche in magrio.

Particolarmente interessanti, perché potrel)hero confermare efficacemente l'esistenza del temporaneo circuito ciclonico del Mar Ligure, i ritrovamenti delle schede n. 158, 159, 152 e 177. Tali gallegrianti, pervenuti in marzo sulle coste del Var, con le lottirlie n. 157 e 162, per successivo orientamento in direzione $\mathrm{SE}$ e $\mathrm{NE}$ attraverso $\mathrm{E}$, avrel)bero raggiunto in aprile quelle nord-occidentali corse (scheda n. 158) per poi rientrare nel Mar Ligure: lo comproverebhero la 159 a Boccaserchio (Pisa) in Magrio, la 152 nelle acque di Nervi (Genovai in giugno e la 177 dinanzi a Nizza in agosto $1 *$ i.

Il ritrovamento in settembre sulla costa nord-occidentale sarda della scheda n. 172 è di dublia interpretazione. Giunta in agosto nelle acque di Nizza, unitamente alla bottiglia 177, esauritosi il predetto circuito ciclonico, essa sarebbe stata ripresa da altra corrente occasionale di completamento, che da $\mathrm{N}$ a $\mathrm{S}$ si estende lungo le coste occidentali corse e sarde; ma tale scheda potreblue anche essere griunta nelle acque spagnole con la 154, e poi, di là, aver seguito il moto ciclonico del Mar Balearico.

4. 1. 5. - Lancio sulla rotta Genova-Porto Torres 129-1.52, ore 15.35 i.

I ricuperi delle hottiglie n. 205 e 207 (marzo-aprile) sul litorale

(n) I questo oruppo apparterrebbe la 197 ricuperata in luglio nel porto di I.ivorno. 


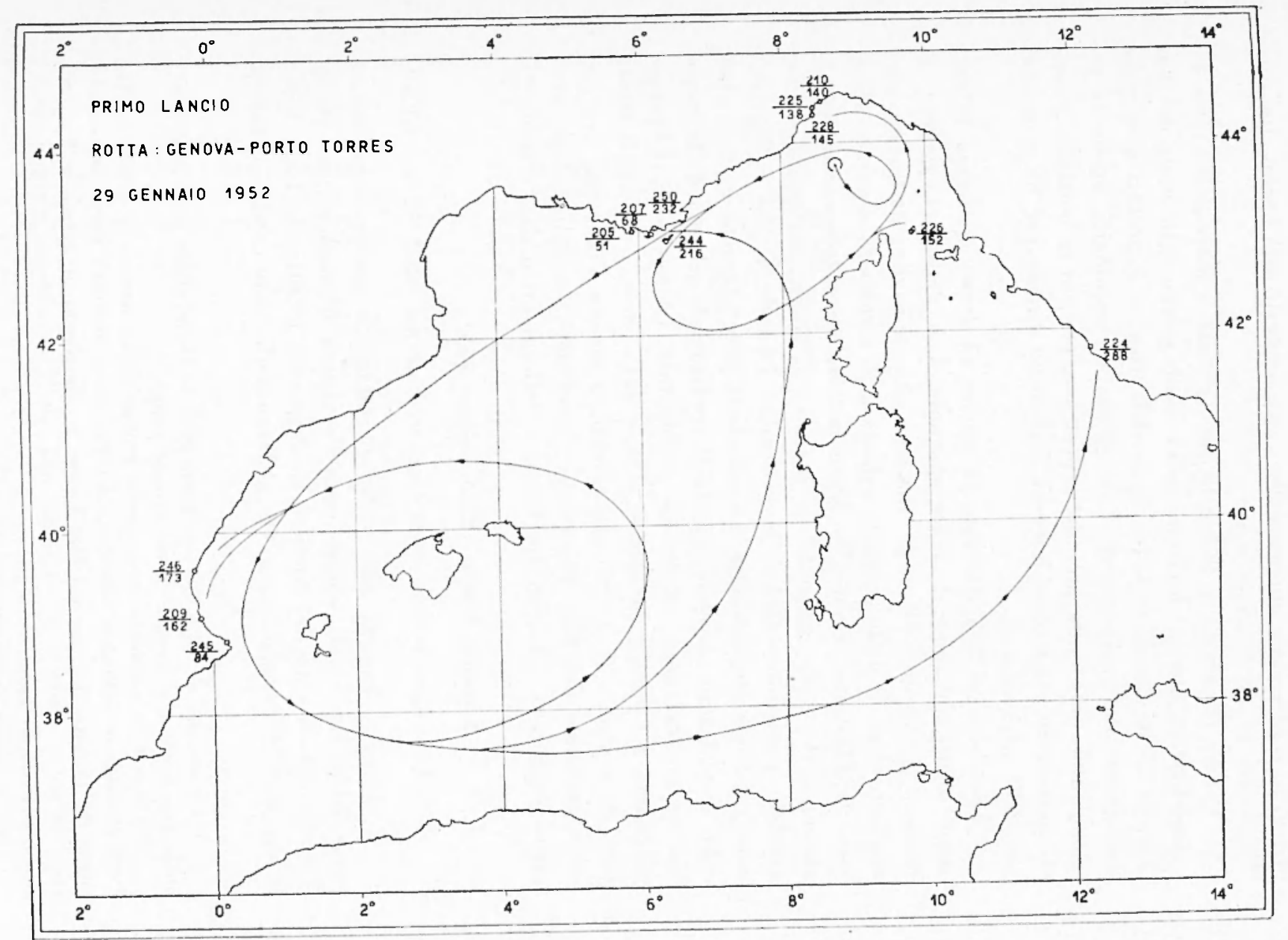

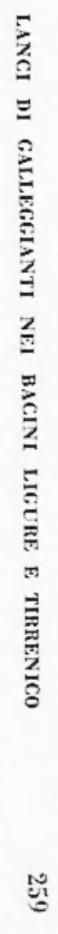


francese confermano ancora nella parte centrale del bacino ligustico l'esistenza di un moto in senso ciclonico.

Poiché la scheda n. 205 e la 162 gettata a mare sulla rotta Livorno-Bastia), furono rinvenute nello stesso giorno sulle coste del $\mathrm{V}$ ar a circa 25 miglia di distanza l'una dall'altra, si e portati a ritenere influenzata dalla già descritta situazione meteorologica anche la regrione interessata dalla presente esperienza, per cui le lottiglie, prima di convergere sulle coste francesi, sarelblero discese per SF e risalite per $\mathrm{NW}$ attraverso $\mathrm{E}$.

La scheda $n$. 245 ritrovata in aprile ad Javea conferma la corrente principale lungo le coste spignole. Con detto gallegriante sareblero pervenute nelle acque ilseriche altre bottiglie: da queste, convogliate verso E dalla corrente atlantica, si sarebbero separate all'altezza dell'Isola di Minorca la 209 e la 246 , ritrovate successivamente ancora sulle coste spagnole in luglio $(*)$. Delle rimanenti, alcume avreblero ragriunto il $\mathrm{Var}$ in settembre (schede n. 244,250 , per mezzo del circuito balearico. La scheda n. 22 , ritrovata in novembre sulle coste laziali, avrehbe seguito il tradizionale schema della circolazione mediterranea nel hacino occidentale. Le bottiglie ricuperate in giugno a Capraia (scheda $n$. 226) e sulle coste occidentali liguri (schede n. 210, 228 "225) attesterebbero ancora l'esistenza del circuito ciclonico del Mar Ligure, ove sarebhero pervenute dopo aver prima raggiunto il $\mathrm{Var}$ in aprile con i galleggianti 11.205 e 207 ( $^{* *}$ ).

4. 2. - Secondo lancio (23-26 marzo 1952).

4. 2. 1. - Lancio sulla rotta Cagliari-Palermo (26-3-52, ore 1 )1.10).

I ricuperi, limitati alle coste salernitane, a quelle sud-orientali sarde ed ai lidi siculi, comprovano lavenuta formazione nel Basio Tirreno del circuito del Nielsen. Galleggianti, giunti alle lsole Folie in maggio (*** risalgono per NW al largo delle coste calabresi sino al

(F) Le bottiglie n. 209 e 246 potrebbero tuttavia essere giunte nelle acque spagnole con la 245 e ricuperate in un secondo tempo.

(*) Non è da escludere che a questo gruppo appartenessero le schede n. 21.4 e 250 ritrovate in settembre sulle coste del Var, dove sarebbero giunte per esautimento del circuito ciclonico del Mar Ligure, a somiglianza di guanto puo esere avvenuto per la scheda n. 177 I lancio sulla rotta Livorno-Batstial rinvenuta nelle accpue di Nizza in agosto.

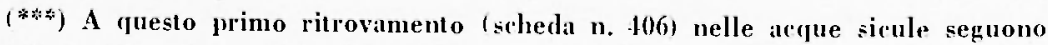
altri ricuperi in giugno (scheda n. 141) e luglio (schede $n, 446,416,426,447,428$ ). 


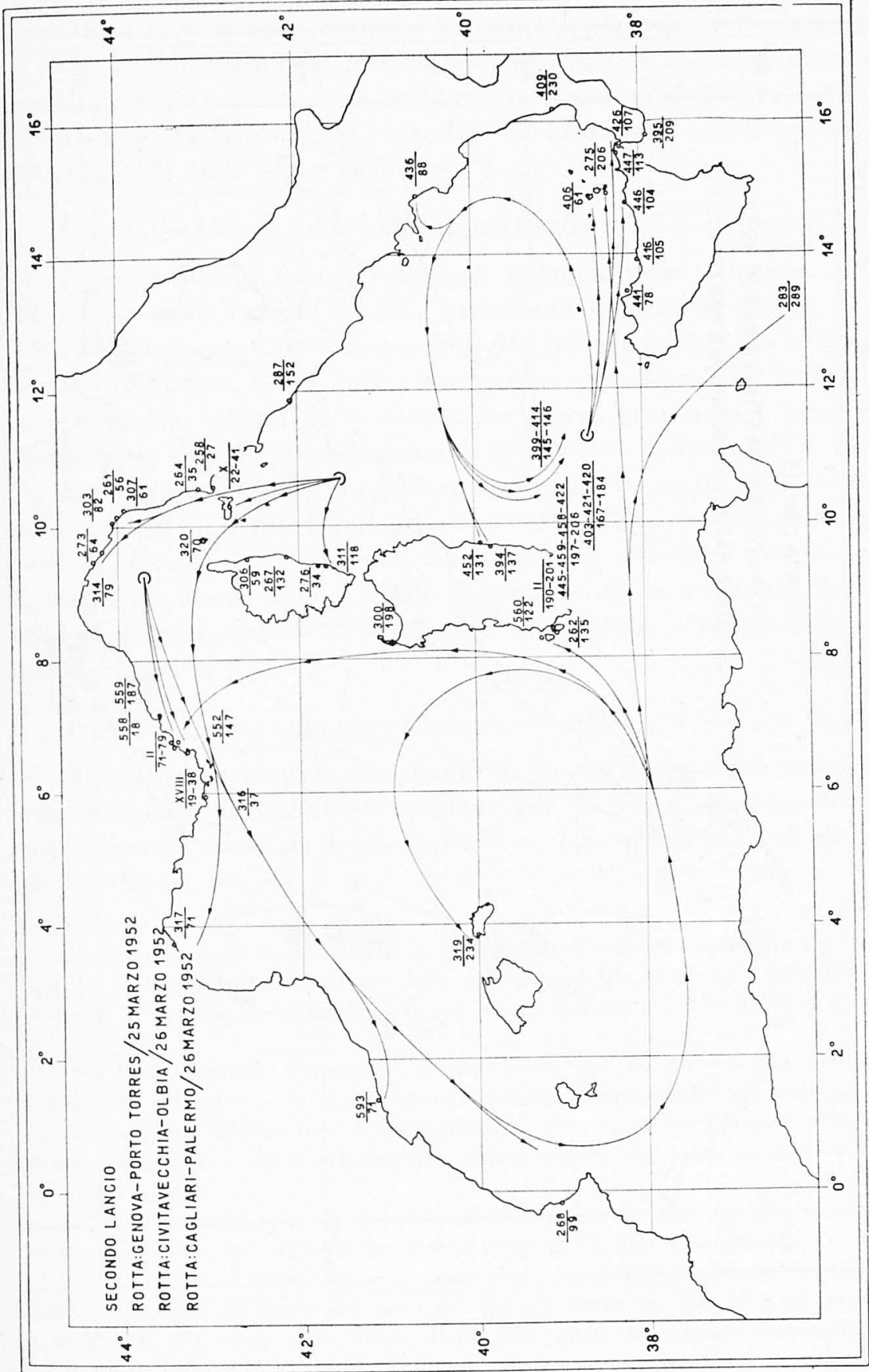




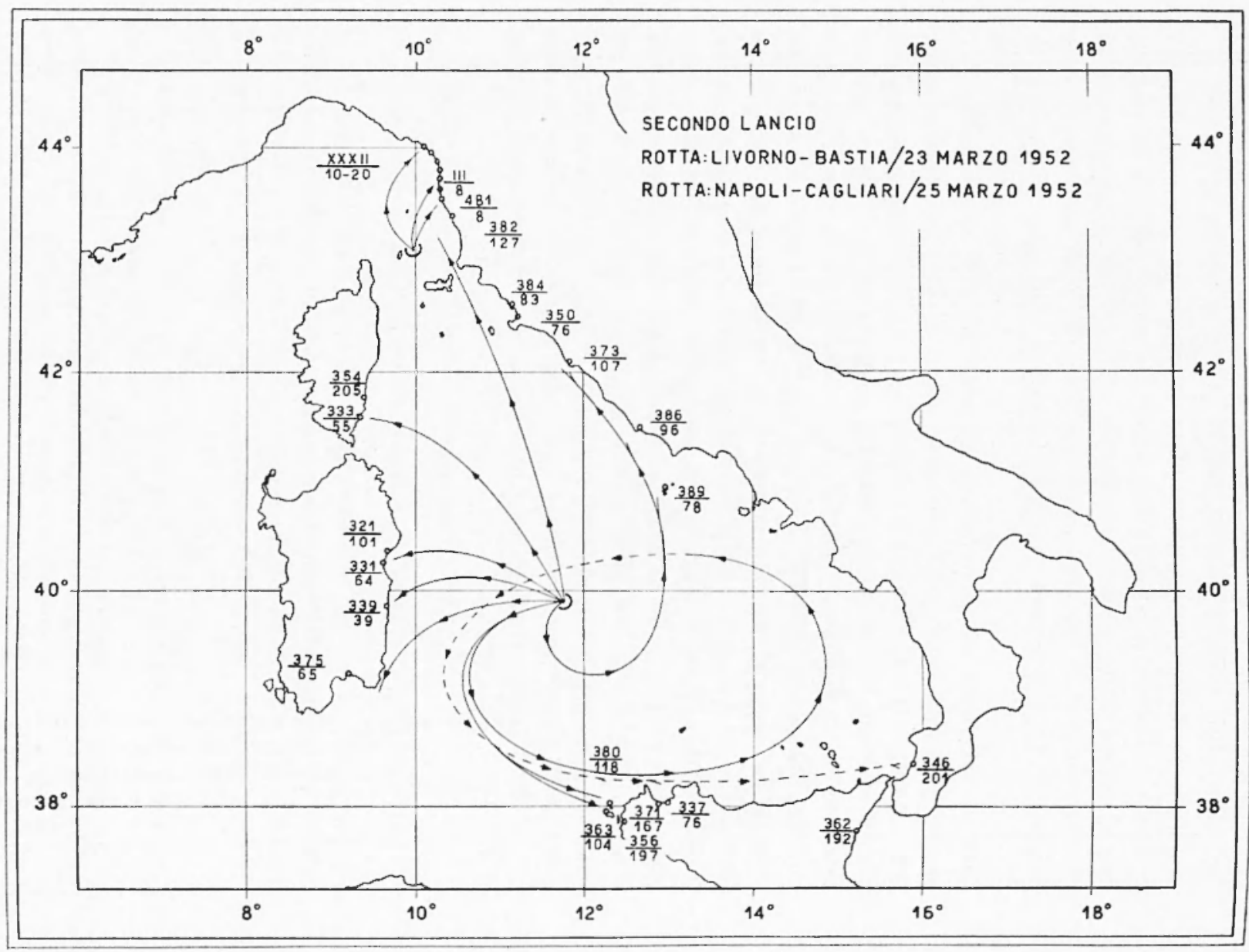


Golfo di Napoli in giugno (scheda n. 436), da dove, con spostamento verso Occidente, alcuni spiaggiano in agosto sul litorale sardo (schede n. 452 (394), mentre altri piegano, con flessione di ampiezza diversa per SW, S e SE e si ritrovano nello stesso mese (schede n. 414 "399), in settembre (schede $n$. 40.3, 421, 420) ed in ottolure (schede n. 445, $459,458,422$, nelle acque siciliane (*).

\section{2. 2. - Lancio sulla rotta Napoli-Cagliari (25-3-'52, ore 21.06).}

I ritrovamenti di magrio sulle coste orientali sarde (schede n. 339, $331,375)$ e corse (scheda $n .3333$ ), unitamente a quelli di griugno (tra il 9 e l'll griugno) a Castellamare del Golfo (scheda n. 337), tra le Isole Pontine ischeda n. 389) e sulle coste toscane (scheda n. 350) indicano -.. per l'ordine geografico e cronologico in cui avvennero i ricuperi medesimi - una diffusione trazionata a carattere ciclonico (**).

Successivamente alla n. 337 spiaggiavano in luglio sulle coste nord-occidentali sicule $i$ galleggianti n. 363 e 380 , mentre altri, seunito il circuito del Nielsen, si ritrovavano in settembre fcome per il lancio di marzo sulla Cagliari-Palermol sulle coste della Sicilia nord-occidentale (schede $n .371,356$ ) ed in ottolse (scheda 11 . 346) su quelle calabre $i^{* * *}$ ).

1. 2. 3. - Lancio sulla rotla Civitavecchia-Olbia (26-3-'52, ore 00.45).

Alcuni gallegrianti, a somiglianza di quanto rilevato per il lancio sulla rotta Napoli-Cagliari, si spostano per W-NW e raggiungono le coste orientali corse in aprile (scheda $n$. 276) e maggio (scheda $n$. 3061 (****).

(*) Per l'esattezza, la hottiglia n. 422 spiaggiò sull'Isola di Formica, nelle Egaldi, e la n. 395 sulla parte estrema della penisola calabra, dopo aver attraversato lo stretto di Messina. In nowmbre, inoltre, venne ricuperatit nel Golfo di S. Eufemia la n. t09.

(**) Delle bottiglie rinvenute successivamente sul litorale toscano ischede n. 384, 382) é incerto se la n. 382 abbia raggiunto Castiglioncello passando ad E o ad W dellisola d'Elba. Con il galleggiante n. 389, raccolto a Ponza, avrebbero inoltre viaguiato $\mathrm{i}$ n. 386 . 373 rinvenuti rispettivamente ad Anzio fgiugnol e Civitavecehia llugliol.

(\$A*) Unitamente alla 316 dovrebbe aver viaggiato la 362 . whe altraterso lo Stretto di Messina, ha raggiunto in ottobre le acque di Riposto (Catania).

(**ask) Di dubbia interpretazione sono $\mathrm{i}$ ricuperi avenuti in giugno $(\mathrm{n} .320$ ) a Capraia, in luglio ed agosto sul versinte orientale corso (n. 311, 267), ed ancori in agosto (n. 28i) sulle coste laziali. Drtti galleggianti, considerata la possibilita che siano stati riportati in circolo dopo un primo spiaggiamento in zone non abi- 
La magrior parte delle bottiglie, avvicinatasi piì celermente al versante meridionale dell'Elba, si scinde in prossimita di questo in due gruppi. Quello orientale segnala, con numerosi ritrovamenti, il suo passaggio in aprile sui lidi elloani, si introduce e supera, ancora nello stesso mese, il Canale di Piombino (schede n. 258, 264) e prosegue in maggio verso $\mathrm{N}-\mathrm{NW}$, parallelo al litorale toscano (schede п. 261,307, 303), per esaurirsi in giugno nelle acque liguri di Riva Trigoso (scheda n. 314). Laltro ramo, trasportato con magriore velocita nella parte centrale della corrente primaria, segnala la sua presenza in maggio sulle coste del Var (scheda n. 316) ed in luglio (scheda n. 268) su quelle iberiche da dove - in prossimita di Capo S. Antonio - viene spinto a $\mathrm{SE}$ e quindi ripreso dalla corrente di origine atlantica. Lo spostamento verso Oriente i confermato dalla scheda n. 262 ricuperata in agosto sulle coste sud-occidentali sarde. Con questa penetrano nel circuito balearico la n. 319, rinvenuta in novemlire nell'Isola di Minorca, c le bottiglie n. 265, 285 e 300 spiagrgiate in ottobre sempre sul versante occidentale sardo. Con la 262 griungevano a SW di Capo Spartivento la n. 275 e la 283 le quali, ritrovate rispettivamente in ottobre tra le Isole Eolie ed in grennaio sulle coste libiche, confermano decisamente il tradizionale schema della circolazione mediterranea.

\section{2. 4. - Lancio sulla rotta Livorno-Bastia (23-3-'52, ore 03.37).}

A circa tredici giorni dal lancio, l'esperimento si esauriva nei numerosi ricuperi avvenuti sulle coste toscane tra Livorno e Marina di Massa. Tale risultato permette tuttavia di illustrare lefletto provocato da un locale moto di deriva.

Il rapido e completo spiaggiamento, avvenuto tra il 31 marzo ed il 12 aprile, trova conferma principale nelle condizioni meteorologiche e specialmente nei venti oceidentali e sud-occidentali, molto frequenti e di particolare violenza in questa area di mare in lale momento $\left(^{*}\right)$. Che il risultato sia dovuto ad un moto di deriva, e come tale a rapido esaurimento, lo dimostra il normale percorso, da Sl per NW. seguito dai gallegrianti lanciati sulla rotta Civitavechia-OH,ia.

tate, potrebbero indicare -- per i mesi estivi - una netta separazione tra le masse d'arcua del Mar Ligure e dell'Alto Tirreno.

(*) I semafori di Gorgona e Capraria segnalano forti venti da W e SW ron massimi di $65(26$ marzo $)$ e 15 (2 aprilel miglia allora. Al riguardo vedasi: Air Ministry, Meteorological Oflce. Weather in the Mediterranean. Local Information. vol. II (I.YI). H. M. Stationery Office, I.ondon 1937. 
4. 2. 5. - Lancio sulla rotta Genova-Porto Torres 125-3-'52, ore 15.20 ).

A 18-20 giorni dal lancio, i numerosi ritrovamenti sulle coste francesi in prossimità del Golfo di Saint Tropez, ad Oriente delle Isole Hyéres, rivelano come $i$ gallegraianti, trascinati dalla corrente più a Settentrione rispetto ai ricuperi della precedente esperienza, siano stati favoriti da venti nord-orientali, di notevole frequenza in primavera, segnalati anche da qualcuno dei ritrovatori delle bottiglie medesime. I gallerraianti n. 593, 560, 552 e 559, oltrepassate le coste meridionali francesi, confermano invece il circuito balearico: ad un primo ricupero in giugno sulle coste spagnole (scheda $n$. 593) ne seguono altri in lugrlio sul versante sud-occidentale sardo (scheda $n$. 560) ed in agosto-settembre (schede $n .552,559$ ) ancora sulla riviera francese.

A detto gruppo appartenevano inizialmente le bottiglie n. $543 \mathrm{e}$ 540 che, riprese da una controcorrente costiera (segnalata in precedenza| all'estremità occidentale del Golfo del Leone, spiagraiavano in griugno sulle coste del $\mathrm{Var}$.

4. 3. - Terzo lancio (29 giugno-2 luglio 1952).

4. 3. 1. - Lancio sulla rotta Cagliari-Palermo (2-7-'52, ore 00.15);

4. 3. 2. -- Lancio sulla rotta Napoli-Cagliari (15-7-52, ore 20.30);

4. 3. 3. -- Lancio sulla rotta Civitavecchia-Olbia (2-7-52, ore 00.30).

I lanci estivi segnalano per i hacini del Medio e Basso Tirreno una mutata situazione dinamica originata prohabilmente dal persistere di regimi barici livellati ad alte pressioni e dal prevalerc di moti occasionali in direzione Sud-Est per venti nord-occidentali.

Gli esperimenti, caratterizzati da assai scarsi ricuperi a oltre sessanta riorni dal lancio, comprovano unitamente ad una notevole dispersione dei rallegrianti, limitata attività dinamica. Delle bottiglie lanciate tra Cagliari e Palemo il 2 luglio, la 708 raggiunge le Isole Eradi in settembre, la 747 i raccolta nelle acque del Mediterraneo orientale in ottobre, mentre i rallegrianti 731 e 738 vengono ricuperati nel Golfo di Patti (Messina) in novembre.

I ritrovamenti relativi al lancio sulla rotta Napoli-Cagliari esulano da considerazione alcuna che non rientri in quelle vià formulate a riguardo dell'arca tirrenica. Un marcato spostamento per SE, denotano le bottiglie lanciate tra Civitavechia ed Olbia, e ricuperate tra le coste nord-orientali sicule e nord-oceidentali calabre: il loro 


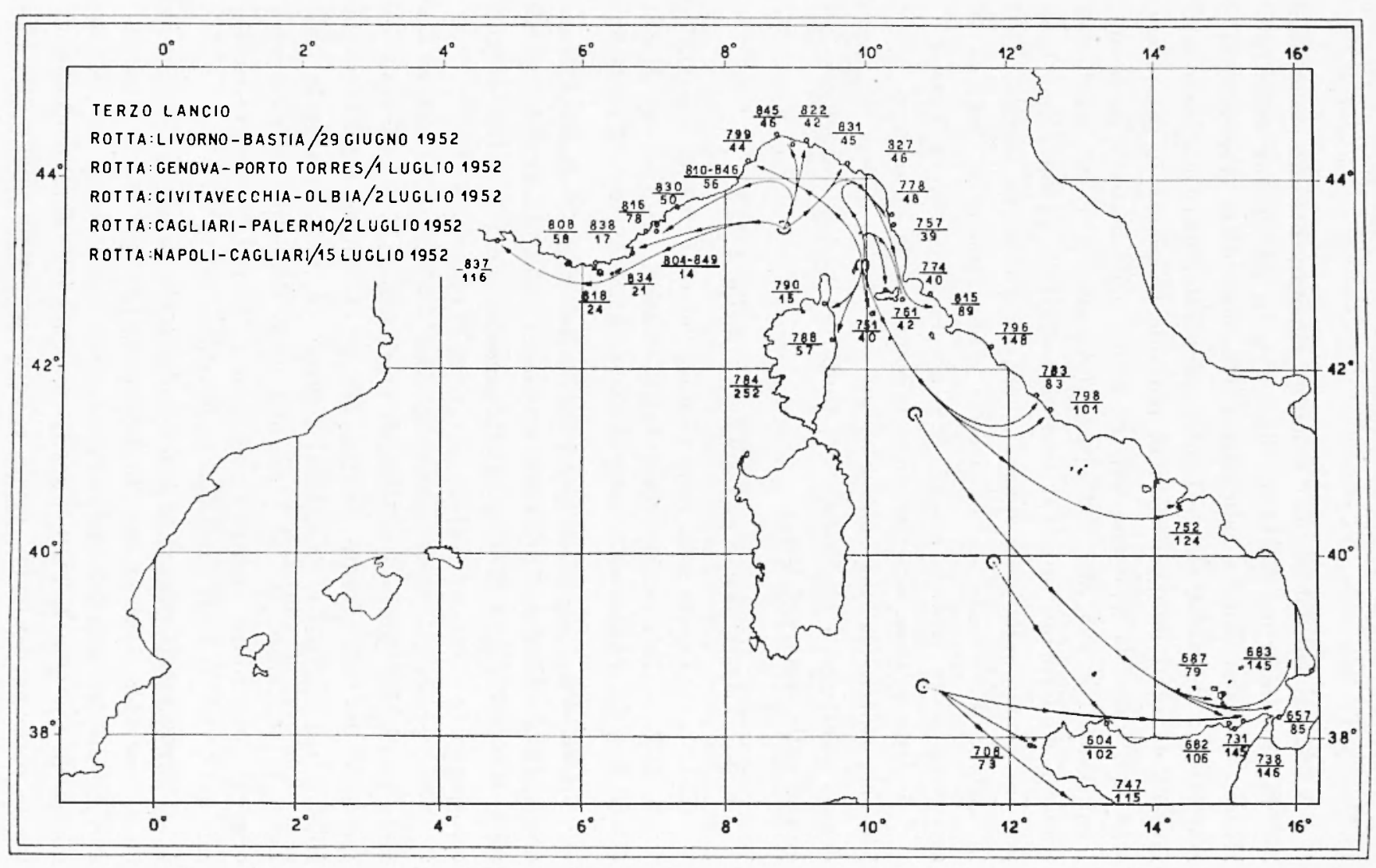


trasporto nel bacino tirrenico avreblue avuto svolgimento a causa della già ricordata prevalenza estiva di venti nord-occidentali (*).

4. 3. 4. - Lancio sulla rotta Livorno-Bastia (29-6-'52, ore 03.30).

Tra Capo Corso e l'arcipelago toscano il moto delle acque presenta - rispetto alle precedenti esperienze - caratteristiche di transizione tra quello del hacino tirrenico a $\mathrm{S}$ e del ligustico a $\mathrm{N}$. Aleune bottiglie, infatti, vengono ricuperate in luglio-agosto sulle coste nordoccidentali corse (schede n. 790, 788), e sull'Isola di Pianosa Ischeda n. 751); contemporaneamente, altro gruppo si porta a Nord della stazione di lancio, ed ancora in agosto un gallegriante viene ricuperato nelle acque di Finale Ligure (scheda n. 7991. Con questa bottiglia si sarebbero spinte a Settentrione le n. 778, 757, 761 e 774 le quali, ricacciate verso Sud da una temporanea controcorrente, sono ricuperate in seguito sui lidi toscani e nel Canale di Piombino (**).

Inoltre, una controcorrente costiera, ritenuta estendersi lungo le spiagrgie italiche da NW per SE, trova conferma limitalamente al periodo estivo-autunnale nei ricuperi avvenuti tra settembre ed ottolse sulle coste laziali (schede n. 796, 78.3, 798, e salernitane (scheda n. 752).

\section{3. 5. - Lancio sulla rotta Genova-Porto Torres (1-7-'52, ore 17.00).}

Nelle acque liguri, a differenza di quelle tirreniche e dell'arcipelago toscano, la corrente tende ancora in direzione delle coste francesi. I 16 grallegrgianti colà ricuperati in Iuglio mostrano invariate, rispetto al lancio precedente, le caratteristiche idrodinamiche del bacino ligustico $\left(^{* * *}\right)$.

(*) Secondo Weather in the Mediterrmean (vol. 11) risultano prevalenti nel Tirreno da giugno ad agosto al largo delle coste dell'Italia Centrale e delle arque centro-sud-occidentali i venti di $\mathrm{NW}$ con frequenza di un giorno su quattro.

(**) Di tale moto contrario, il Boscovich (vedi Platania, op. cit., pag. 87) rilevò la costante presenza tra Livorno ed il Golfo della Spezia. Uzielli tvedi Platania. op. (it., pag. 143, nota 2) to confermerebhe per i mesi di luglio-agosto sulle coste liguri comprese tra La Spezia e Sestri Levante.

(***) I ricuperi dei galleggianti sulle coste francesi ebbero inizio a 14 giorni dal lancio. similmente a quanto avenuto nella precedente esperienza di marzo. che registró i primi ritrovamenti, sempre sul litorale francese. 18 giorni dopo che le bottiglie erano state lanciate a mare. Inche per il presente esperimento coloro che effettuarono i ricuperi segnalano forti venti orientali, che "Weather in the Mediterranean " definisce caratteristici in estate ma non con la stessa intensita primaverile ed invernale. 
Altre hottiglie si distribuiscono verso Occidente lungo le coste meridionali, sino ad esaurirsi nel Golfo del Leone. Dalla stazione di lancio, alcuni galleggianti si avvicinano alle spiaggie francesi dopo una più mareata inflessione verso $N$, in direzione del Golfo di Genova, come indicato dai ricuperi delle schede n. 830, 810, 846 e 816 in agosto-settembre a $\mathrm{S}$. Jean Cap Ferrat, a Cannes ed alle Isole Lerin.

A questo gruppo appartenevano inizialmente le bottiglie ricuperate ancora più a $N$ nelle acque costiere ligustiche $(822,845,831$ e 827 ), e la n. 815 ritrovata a settembre sulle spiaggie grossetane, trascinatavi da temporanea controcorrente costiera.

4. 4. - Quarto lancio (2-6 novembre 1952).

4. 4. 1. - Lancio sulla rotta Cagliari-Palermo (6-11-'52, ore 00.30).

Particolare situazione depressionaria gravitante sul Basso Tirreno determina il rapido convergere dei gallegrianti sull'Isola di Favignana e sulle coste occidentali sicule.

Delle bottiglie lanciate a mare il 6 novembre, e che hanno subito l'influsso di venti occidentali e nord-occidentali tra l'8 ed il 12 (", si inizia infatti il ricupero con la 995 al largo del Faro di Punta Favignana il 13 , seguito in breve tempo da numerosi ritrovamenti (dieciassette).

Come per l'esperimento di marzo sulla rotta Livorno-Bastia, nel presente si assiste alla formazione di un moto da deriva che trascina in direzione $\mathrm{SE} i$ gallegrgianti, e temporaneamente annulla o sposta sensibilmente a mezzogiorno il ramo della corrente atlantica che regolarmente dalle coste settentrionali siciliane decorre per NW parallelo al litorale italico.

Nessun ricupero, infatti, avviene nelle acque tirreniche, e solo pochi galleggianti $(n .986,969,988)$ raggiungono il bacino orientale mediterraneo.

4. 4. 2. - Lancio sulla rotta Napoli-Cagliari (4-11-52, ore 24.00).

Le condizioni meteorologiche segnalate, e comuni a tutto il Basio T'irreno, provocano - similmente a quanto i avvenuto fer il contem-

1*) Tra il 7 ed il 12 novembre 1952, i semafori dell'Isola di Favignana e di Capo Carbonara registrano, per il vento, quali medie giornaliere le seguenti dire. zioni e velocità :

Capo Carbonara: W $50-\mathrm{NW}+1 . \mathrm{NW}+10 \cdot \mathrm{IV}, 36-\mathrm{W}+18 \cdot \mathrm{W} 36$

Isola Favignana: $S W 37 \cdot N W 61 \cdot \mathbb{W} 31 \cdot N W \cdot 7 \cdot N W 53 \cdot W 32$ 


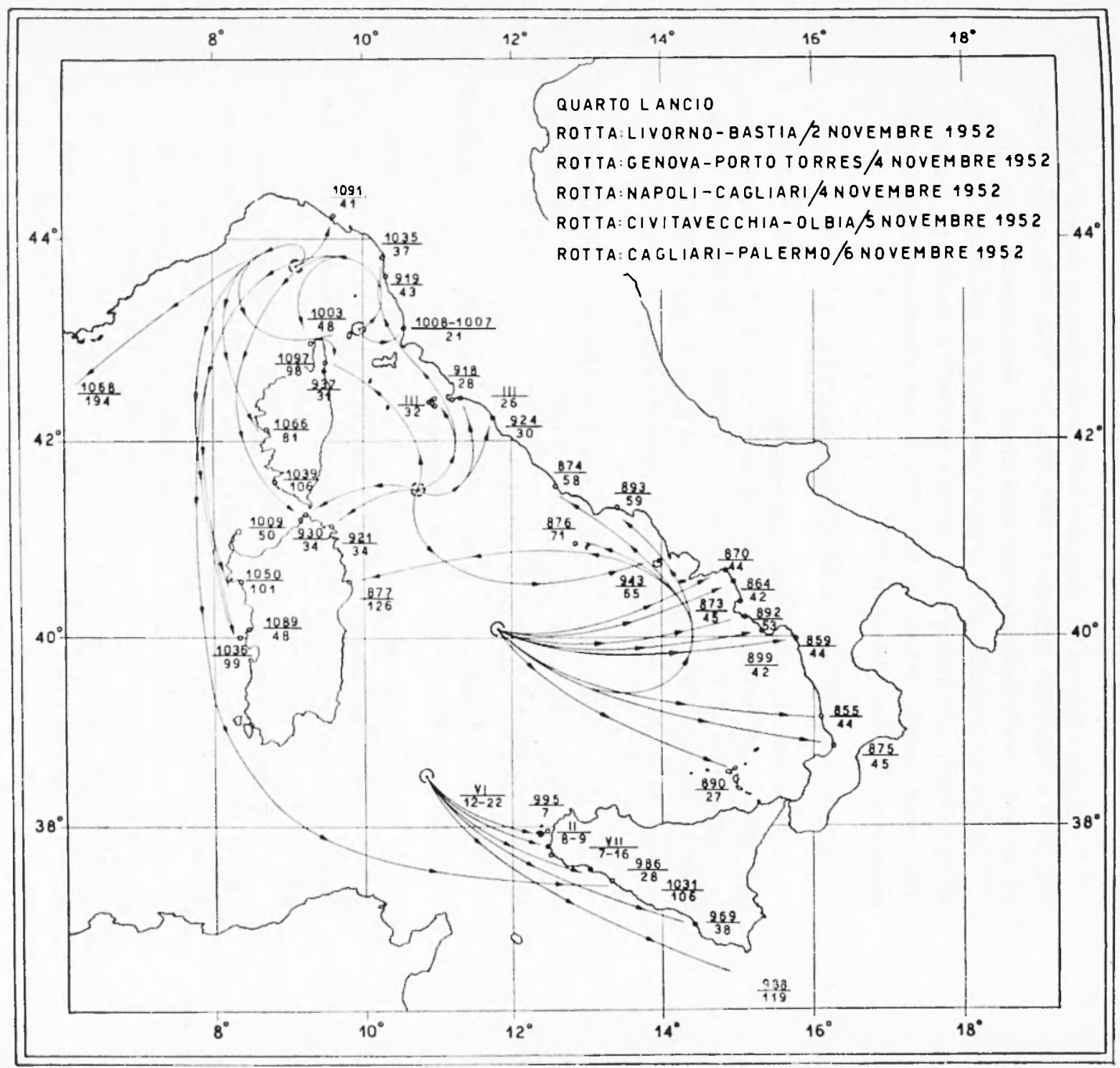


poraneo lancio sulla rotta Cagliari-Palermo - un mareato spostamento dei galleggianti verso $\mathrm{SE}$, tendente gradualmente ad ampliari per E-SE con l'avvicinarsi di essi alle coste calabresi e partenopee.

I ricuperi effettuati in dicembre sul litorale calabro (schede $\mathbf{n} .875$, 85.5, 859) e campano (schede $n$. 899, 892, 87.3, 864, 870), confermano un diretto e contemporaneo investimento di detti litorali (*).

Da queste bottiglie, altre si sarebhero separate all'altezza del Golfo di Salerno per iniziare una risalita verso $\mathrm{N}$ esauritasi in gennaio sulle coste laziali (schede $n .874,893$ ), o per seguire parzialmente il circuito di Nielsen (scheda n. 877).

4. 4. 3. - Lancio sulla rotta Civitavecchia-Olbia (5-11-'52, ore 00.30).

I ritrovamenti sulle coste tosco-laziali (schede $n .902,90.3,935$. 918, 924), e sull'Isola del Girlio (schede n. 9.34, 947, 929), confermano - unitamente ai ricuperi, sempre di dicembre, a Nord dell'Isola d'Elba (scheda n. 919) e nella Penisola di Capo Corso (scheda n. 937) - come le acque tirreniche tendano nuovamente a spostarsi in direzione NW $\left(^{* *}\right)$.

Tre altri ricuperi, invece, più a Sud della stazione di lancio, sulle coste nord-orientali sarde ed all'Isola d'Ischia, non concordano ron la maggioranza dei ritrovamenti.

4. 4. 4. - Lancio sulla rotta Livorno-Bastia (2-11-'52, ore 03.20):

4. 4. 5. - Lancio sulla rotta Genova-Porto Torres (4-11-'52).

I ricuperi indicano per le acque liguri e dell'arcipelago toscano il prevalere di una situazione barica diversa da quella rilevata per il Medio e Basso Tirreno da lanci contemporanei sulle rotte Napoli-Cagliari e Cagliari-Palermo.

Le bottiglie che inizialmente ed in parte si spostano verso $\mathrm{Y}$ (1035, 1091), convergono in seguito - in contrasto con il normale decorso della corrente - sulle coste occidentali corse e sarde.

I successivi ricuperi di dicembre nelle acque di Centuri, di S. Teresa di Gallura e di S. Vero Milis (schede n. 1003, 1009, 1089) confermano per i galleggianti dei due esperimenti l'avvenuto spostamento ad Occidente nelle acque baleariche.

(4) Degli otto ritrovamenti sulle coste calabre e campane sette sono avvenuti Ira il 16 ed il 19 dicembre, l'ottavo il 27 dello stesso mese.

(*) Permane l’incerteza se la 919 abbia raggiunto il litorale livornese attraverso il Canale di Piombino o passindo tra le coste elbane e le corse. 
La bottiglia n. 1031, oltrepassate le coste occidentali sarde e presa dal ramo della corrente atlantica, viene raccolta in febbraio sul versante meridionale siculo.

I ritrovamenti di gennaio e felbloraio sulle coste occidentali corse e sarde (schede $1.1066,1036,1050,1039$ ) inducono a ritenere che le bottiglie trascinate a Sud siano state riprese gradualmente dalle acque del moto balearico, e quindi trascinate verso Nord e ricuperate in località più settentrionali di quelle ove avvennero i primi ritrovamenti; oppure che esse abbiano ragriunto dette coste, dopo più ampia inflessione nel Golfo Ligure.

\section{V. - Considirazioni generali.}

\section{1. - Risultati generali dei lanci.}

Galleggianti lanciati ove il Tirreno raggiunge la massima estensione possono a lungo vagare con regimi livellati ad alte pressioni (III lancio: Napoli-Cagliari, Cagliari-Palermo), od avvicinarsi alle coste, se favoriti da particolari condizioni meteorologiche (I lancio: Napoli-Cagliari, Cagliari-Palermo), e proseguire quindi secondo la tradizionale corrente litoranea.

Al contrario, là dove le coste insulari sarde e corse da un lato e tosco-laziali dall'altro limitano la estensione dell'area tirrenica, il moto delle acque aumenta di velocita verso $N W$; non sono tuttavia esclusi moti di deriva collaterali (I, II, IV lancio: CivitavecchiaOll,ia).

Le acque dell'arcipelago toscano possono partecipare alla circolazione del Mar Ligure ed a quella del Tirreno (III lancio: LivornoBastia), oppure partecipano senz'altro a quella del Mar Ligure sia per effetto dei venti connessi ad una stabile situazione barica (I lancio), sia per l'influenza di campi barici locali (IV lancio). L'Alto Tirreno presenta quindi insieme alle acque Elbane una certa affinita di comportamento, anche se parzialmente dovuta alla predominanza stagrionale dei venti, col Mar Ligure.

I ricuperi, inoltre, possono essere correlati o ad un'unica situazione barica interessante i due bacini (I lancio) o a contemporanee situazioni che diversamente agiscono sulle acque dei medesimi (IV lancio: Livorno-Bastia, Genova-Porto Torres; IV lancio: Napoli-Cagliari, Cagliari-Palermo); la corrente può subire temporaneo annullamento per forti venti (II lancio: Livorno-Bastia; IV lancio: Carliari-Paler- 
mo), o ricavarne aumenti di velocità quando prevalgono nella stessa direzione.

A Nord dell'arcipelago toscano, in cui si hanno questi regimi di transizione, nel periodo estivo, il moto delle acque da SE per $N W$ (III lancio: Genova-Porto Toresh, continua permanentemente mentre a Sud, nelle acque tirreniche, si interrompe assumendo direzione contraria a quella avuta in precedenza (III lancio: CivitavecchiaOlbia).

\section{2. - Il regime delle correnti e il regime dei venti.}

Sembra importante rilevare come il regime dei venti $(*)$ sia sufficiente a spiegarci la diversa distribuzione dei galleggianti nelle quattro stagioni, anche nellipotesi che nelle acque liguri e tirreniche non esista alcuna corrente.

Infatti d“inverno, nel Tirreno, i galleggianti trascinati dai venti occidentali e nord-occidentali ad Oriente, ove prevalgono quelli a

(*) Da "Weather in the Mediterranean", clie riporta una completa bibliografia sull'argomento, risulta cosi sintetizzalo il regime dei venti nei mari Ligure e Tirreno:

Inverno - Nel Mar Ligure la prevalenza spetta ai venti settentrionali, specie di NE. Ina distribuzione piutosto uniforme appaiono avere nel Tirreno, dove a Settentrione prevalgono forti venti nord-orientali frequenti ma non forti sono anche i venti di SE) contemporanei a quelli di $W$ e di $N W$ nella parte sud-occidentale del bacino e tra $\mathrm{SE}$ e $S \mathrm{~W}$ in quella sud-orientale.

Primavera - Nel Mar Ligure i venti prevalenti sono compresi tra NW e SE attraverso SW, essendosi sensibilmente ridotta la frequenza di quelli di NE. Le direzioni predominanti sono $\mathrm{NW}$ e $\mathrm{SE}$, ma non di gran lunga più frequenti rispetto alle altre di $S$ e $S W$. Yel Tirreno persiste la condizione invernale, senza aversi una definita direzione drminante; le frequenze maggiori sono $\mathrm{NW} e \mathrm{SF}$, parallelamente alle coste. benclé a Settentrione si abbia qualche ecresso da NW, W e S e nella parte rentrale del bacino i venti piu frequenti siano NW, W e SW. La loro forza diminuise sensibilmente rispetto a quella registrata in inverno.

Estate - Nel Mar Liqure scarsi ed incostanti i venti da E e NE, prevalendo quelli tra $S$ e $N$ allraverso $W$ con ecressi di $S W$ e $N W$ rispetto a $W$. Nel Tirreno, al largo delle coste dell'Italia centrale e nelle acque centro e sud-ocridentali predominano i venti di NW. A Nord essi si distribuiscono principalmente nella stessa direzione riscontrata per il Ligustico; nella parte sud-orientale non si la una definita prevalenza e si riscontra qualche eccesso di $\mathrm{NW}, \mathrm{N}$ e SE. La forza del vento diminuisce sensibilmente.

Autumno - Nel Mar Ligure i venti predominanti sono di SE diminuendo gli estivi ed aumentando quelli da SE, E. NF. Nel Tirreno si ritorna ad una distribuzione sui quadranti piuttosto uniforme con eccessi da $N W$ e $W$ nelle zone cen. trali ed occidentali, da SE a SW nelle zone suderientali e da NW e SE al largo delle coste dell'Italia Centrale. 
componente meridionale, tendono a Settentrione parallelamente alle coste italiche; nel Mar Ligure un regime anemonico a prevalenza nordorientale spinge le bottiglie in direzione $\mathrm{SW}$ in conformità di guanto avviene ammettendo la esistenza della corrente.

In primavera, sebbene meno marcata, si riscontra nel Tirreno la medesima vicenda, mentre nel Golfo Ligure $i$ venti con componente da $S$ spingono a $\mathrm{N}$ i galleggianti che riprendono il loro percorso per SW con venti di NE, ora limitati alla parte più nord-occidentale del bacino ligrustico ed alle acque rivierasche francesi.

In estate, permanendo nel Mar Ligure, in linea di massima, la condizione primaverile, le bottiglie convergono ancora sulle coste del Var. Nel Tirreno e, parzialmente nelle acque dell'arcipelago toscano, dominano venti di NW che provocano lo spostamento dei gallegrianti in direzione SE.

In autunno le condizioni meteorologiche tendono nei Mari Ligure e Tirreno ad identificarsi gradualmente con quelle invernali, benche, per le acque ligustiche e dell'arcipelago toscano, i nostri risultati non lo confermino nettamente.

Pertanto, venti con predominanza e forza variabili stagionalmente e caratteristici di singole aree di mare provocano moti di deriva nel senso della corrente litorale in inverno, e di contraria direzione in estate. L'incostanza che tale situazione lascia supporre si rivela ruando altri moti di deriva, originati da venti di quadranti diversi. provocano la scompara temporanea della corrente principale od un suo rallentamento, oppure l'aumento della sua velociti se prevalenti nella stessa direzione.

5. 3. - D'altra parte le ricerche talassografiche hamno ormai dimostrato come nel Tirreno penetrino masse d'acqua separatesi dalla corrente atlantica in prossimità del Canale di Sicilia.

Dette acfue, di minor densiti rispetto a quelle tirreniche. raggriungono le coste settentrionali sieule da dove convergono sul bolfo di Napoli: secondo Nielsen (13), in tale area, parte di esse per successivo spostamento in direzione WSW-SW originano il circuito del Basso Tirreno, proseguendo le rimanenti verso NW parallele al litorale ilaliano.

Gli scarsi dati idrografici a nostra disposizione sulle caratteristiche fisico-chimiche delle acque tirreniche non rivelano se il moto si esaurisca progressivamente, oppure se esso persista - con limitata estensione ed intensità - sino ad innestarsi a SW del Golfo di Geno- 
va nel circuito balearico a completamento di una circolazione ciclonica del bacino occidentale mediterraneo $(*)$

5. 4. - Comprovata l'esistenza della corrente litorale sulle coste napoletane, si assiste ad una diminuzione della sua potenzialiti dinamica, per formazione del circuito del Nielsen e per l'ampia estensione che il Tirreno presenta ancora a $N$ delle coste campane. Se non intervenissero cause meteorologiche, le aeque tenderchbero ad avere una piu precisa direzione ed una maggiore velocitì durante il loro lento e costante progresso verso il $\mathrm{N}$, con la progressiva limitazione della superficie tirrenica in prossimita dellarcipelago toscano, per giungere quindi nelle acque ligustiche. Poiche pero in inverno ed in primavera prevalgono regimi di vento nel senso della litorale, i certo che essi rinforzano la corrente originaria conferendole un più preciso orientamento ed una maggiore velociti. I nostri esperimenti confermano tuttavia come particolari situazioni hariche possano temporaneamente annullare il moto di essa nel periodo invernale.

Le medesime ragioni giustificano in estate, con la prevalenza di venti nord-occidentali, l'estinguersi della corrente, le segnalate discontinuiti dinamiche tra le acque liguri e tirreniche e l'instaurarsi di un moto di deriva in senso contrario. Tnfatti le coste francesi, raggriunte da galleggianti lanciati in luglio nelle acque liguri, non sono toccate da altre bottiglie lanciate contemporaneamente in quelle dell'arcipelago toscano; mentre gli esperimenti invernali e primaverili, danno ricuperi sulle coste gallo-ispaniche anche di hottiglie gettate a mare sulla rotta Civitavechia-Olbia. Il lancio estivo eseguito su questa rotta, come pure quelli eseguiti da punti piò meridionali, dimostrano l'avvenuta formazione di un moto da deriva contrario alla corrente principale.

A particolari condizioni meteorologiche i da attribuirsi, poi, la temporanea formazione del circuito ciclonico chiuso del Mar Ligure. Frequenti depressioni gravitanti nel periodo invernale (**) $^{*}$ nelle acque

(*) Al riguardo sono in via di elahorazione $i$ dati raceolti nelle acclue liqustiche e dell'arcipelago toscano per mezzo di crociere idrografiche promosse dal Centro Talassografico Tirreno (Trottı L., Prima crociera talassografica del "Ro. busto " nel Mar Ligure ed Aho Tirreno. Centro Talassografico Tirreno. Pubbl. n. 7. Genova 1950$)$.

(**) I] Bollettino quotidiano di informazioni del Servizio Meteoroloyico dell'Aeronautica segnala al riguardo, nella predetta area di mare, la presenza di numerose situazioni depressionarie tra gennaio a marzo. 
liguri ad E e W di Capo Corso e tra questo ed il Golfo di Genova spiegano come galleggianti dei lanci invernali (I lancio: GenovaPorto Torres, Livorno-Bastia) in prossimità delle Isole Hyéres possono convergere nuovamente nel Mar Ligure, radendo il litorale nordoccidentale corso.

\section{VI. - Conclusioni.}

Le acque d'altura dei hacini ligure e tirrenico sono influenzate in modo notevole dai regini di vento prevalenti stagionalmente, $i$ quali, generando moti da deriva in direzione concorde o contraria alla nota corrente litoranea, ne alterano la fisionomia; ciò avviene anche in occasione del passaggio delle singole depressioni. Senza voler attribuire i moti, dedotti dai nostri esperimenti, ad un mese piuttosto che ad un altro, perché essi dipendono sensibilmente dalle condizioni meteorologiche, si può affermare che nei diversi momenti stagionali avvengono particolari mutazioni del moto delle acque superficiali che si possono cosi schematizzare.

La corrente litorale parallela alle coste tirreniche ha in inverno carattere permanente e può raggiungere, con graduale spostamento verso Nord-Ovest, attraverso le acque dell'arcipelago toscano, la parte centrale del Mar Ligure, quando le acque di origine atlantica convogrliate nel Tirreno non si dipartiscono in prossimità del Golfo di Napoli per originare il circuito del Nielsen, favorite nel loro cammino verso NW da venti predominanti nella stessa direzione e che ne aumentano la velocità a Settentrione.

In primavera, contemporaneamente alloriginarsi del suddetto circuito ciclonico del Basso Tirreno, e di altro circuito interessante il Mar Ligure, la litorale si interrompe al largo del Golfo di Napoli, attenuandosi al tempo stesso la continuità dinamica tra le acque dell'arcipelago toscano e quelle ligustiche.

Nelle prima estate la separazione tra il Mar Ligure ed il Tirreno ¿ quasi completa, rimanendo unica via di comunicazione il Canale di Piombino. Le acque ligustiche sono interessate al relativo circuito; nel hacino tirrenico si instaura gradualmente un moto da deriva in direzione SE per venti nord-occidentali.

Nel periodo estivo ha pieno svolgimento nel Basso Tirreno quello del Nielsen: nell'Alto e nel Medio domina la deriva in precedenza segnalata. Le acque dell'arcipelago toscano tendono parzialmente a 
spostarsi nel Golfo ligure, dove moti stagionali si dirigono verso le coste francesi. Al tempo stesso nella parte sud-orientale del bacino ligustico, all'altezza del Golfo della Spezia, si origina una controcorrente costiera che volge verso Sud parallela ai lidi toscani sino al Canale di Piombino.

In autumno le acque tirreniche tendono gradualmente a ricongiungersi a quelle ligustiche; il circuito del Nielsen termina con la prevalenza invernale di venti sud-orientali e sud-occidentali che provocano la rottura di detto circuito ed il rafforzamento della corrente litorale. Nel corso dell'anno una controcorrente costiera si estende dal Canale di Piombino ai lidi settentrionali siculi dove i nostri ricuperi la confermano più concretamente.

6. I. - Al termine di queste esperienze che hanno favorito per alcuni momenti stagionali una visione di insieme e contemporanea della situazione dinamica dei Mari Ligure e Tirreno, si spera che altre seguano più frequenti per una completa conoscenza di tale situazione.

Per meglio delineare la incostante continuità dinamica tra il Mar Ligure ed il Tirreno si ritiene utile che altri lanci alhbiano luogo immediatamente a Sud dell'arcipelago toseano. Gli esperimenti futuri. da eseguirsi sulle stesse rotte, dovranno inoltre essere accompagnati da lanci costieri e di altura, sia per limitare l'ampiezza della radente e del moto costiero ad essa contrario, sia per confermare le deduzioni suggerite dai risultati di questa prima ricerca.

Esprimo la mia gratitudine al Prof. M. Tenani per il Suo interessamento, e ringrazio il Centro Talassografico Tirreno che ha reso possilile, con l’aiuto finanziario prestatomi, la presente ricerca.

Genova - Centro Talassografico Tirreno - Gingno 195.3.

\section{RIASSUNTO}

L'A., poiche le precedenti esperienze furono effettuate saltuariamente in vicinanza delle coste" con scarso numero di galleggianti, espone le ragioni che hanno indotto ad eseguire nuovi esperimenti in arque di altura e contemporanei in diverse aree dei Mari Ligure" Tirreno.

All'esame statistico dei ricuperi ottenuti dal lancio di oltre 1000 
bottiglie in quattro momenti stagionali, l'A. fa seguire un esame cartografico e cronologico dei medesimi.

Le considerazioni e le conclusioni dedotte mettono in evidenza il comportamento della corrente litorale delle acque superficiali liguri " lirreniche in dipendenza dei campi barici edei regimi di vento prevalenti stagionalmente.

\section{$S U M M A R Y$}

The author, since the preceding experiments were carried out in a tentative way near the coust and with fen flouts, explains the reasons which have induced him to carry out new experiments at limes, in high seas and contemporaneously in various areas of the Ligurian and Tirrenian seas.

After a statistical examination of the recovered samples from the posting of more than 1000 bottles at four seasonal times, the author follows ont a graphical and cronological analysis of these samples.

The considerations and the conclusions dratn, show the bchavior of the costal current of the surface waters and the dependence on the pressure areas and on the wind patterns seasonally prevalent.

\section{BIHLIOGR IFIA}

(1) DHILA Cnock N.: Lanci di galleggianti per lo studio delle correnti superficiali nel bacino tirrenico (nota preliminare). La Marina Mercantile, anno V, n. 6 , Genovi 1952.

(-) Manixititi O. Putavia G.: Della correme litorale del Mediterraneo con particolare riguardo alla costa oriemale della Sicilia. Men. Geogr. n. 4, 1908.

(3) A. C.: Il "Principe di Udine " e la corrente del Golfo di Genova. La Mar. Merc. Ital., anno VI, n. 127, 1908.

(1) Manivet.t O.: Sulla corrente litorale nel Gollo di Genova. Riv. Geogr. ltal., anno XVI, fises. VI. 1909.

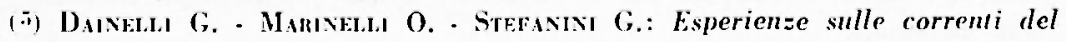
Tirreno. Mem. Geogr., n. 22, 1913.

(i) Mans l.: Lanci di galleggianti per lo studio delle correnti superjiciali nel Mar Ligustico eseguito nel 191\%. Boll. R. Com. Talass. Ital., n. 29-30. Venezia 1914.

(i) Babassfrox, V. - Stemaxis G.: Risulhati di una serie di esperienze sulle correnti del Tirreno. Atti VIII Congr. Geogr. Ital., Firenze, 28 marzo - 6 alprile 1021 , vol. II. 
(s) Marivi I.: Risultati dei lanci di galleggianti per lo studio delle correnti superficiali del Mar Ligure eseguiti negli anni 1914, 1920-22. Atti Soc. Ligust. Sc. Lett_ vol. VI, Genova 1927.

(9) Dainflit G. - Marineldi O. - Stefanini G.: A proposito di una nuova serie di osservazioni sulle correnti nel Golfo di Genova. Riv. Geogr. Ital., anno XXII. fasc. II, 1915.

(10) Troтti L.: Ricerche idrografiche sulle acque costiere ligustiche comprese tra lisola Palmaria e Capo Mele. Parte I. Introduzione, osservazioni meteorologiche e fisico-chimiche. Centro Talass. Tirreno, Pubbl. Spec. n. 8, Genova 1951.

(11) Platavia G.: Experiments uth drift bottles. Rep. Dan. ocean. exp. 19081910 to the Mediterramean adj. seas, n. 7, 1923.

(12) Marineld G.: Esperimenti e rilievi sulle correnti superficiali del Tirreno. Ann. R. Ist. Sup. Nav,, vol. I, fasc. I. Napoli 1932.

(13) Nisuses J. N.: Hydrography of the Mediterranean and adjacent uaters. Rep. Dan. ocean. exp. 1908-19l0 to the Mediterranean adj. seas. n. l, 1912.

(14) Sснотт G.: Die Gewïsser des Mittelmeeres, vorzugsweise nach den Arbeiten des dänischen Forschungsdamplers "Thor" 1908-10. Ann. der Flvdr. und mar. Meteor. Deutsche Seewarte, Hamburg. Bd. 43, 1915.

(1.) Ministero Difesa-Areonautica. Ispettorato delle Telecomunicazioni e della assistenza al volo: Bollettino quotidiano di informazioni del servizio meteorologico dell'Aeronautica. Istituto Poligrafico dello Stato, Roma 1952.

(16) Air Ministry, Meteorological Office: Wreather in the Mediterranem. Vol. I, Vol. II (I.VI). H. M. Stationery Office, London 1937.

(1i) Trotti L.: Prima crociera talassografica del "Robusto" nel Mar Ligure ed Alto Tirreno. Centro Talassografico Tirreno. Pubbl. n. 7, Genova 1950.

\section{NOT A}

Osservazioni relative alla lettura dei disegni.

I luogi ove avvennero i recuperi sono segnalati sulle rarte geograficlie con un cerchietto ed una frazione in vicinanza di esso: il numeratore indica il numero del galleggiante, il denominatore $i$ giorni intercorsi tra lancio e ricupero.

Quando il numeratore è espresso in caratteri romani, esso rappresenta il numero complessivo delle bottiglie ricuperate in una località oppure nella stessa zona: nel raso di ritrovamenti particolarmente indicativi, esso è formato dai numeri delle singole bottiglie. Il denominatore allora, riporta il numero dei giorni trascorsi tra lancio e ricupero per il primo e l'ultimo ritrovamento effettuati nella zona medesima.

Più ricuperi nella stessa localiti, od in immediata vicinanza di essa, sono sem. pre contrassegnati da un unico cerchietto.

Sono escluse dalle carte, bottiglie ritrovate dopo un lungo periodo di tempo o di scarsa importanza nellavvalorare la direzione delle correnti gia messa in evidenza da più numerosi ritrovamenti. 
Le curve rappresentate hanno, di massima, valore indicativo e non sempre se. guono i galleggianti sino al luogo di ricupero perché incerta e difficile è l'interpretazione degli spiaggiamenti, una volta che le bottiglie sono giunte in prossimita delle coste, dove risentono l'influenza dei rilievi costieri e di locali moti di deriva.

Altre curve sono tratteggiate od interrotte prima di avvicinarsi alle coste per evitare sovrapposizioni che potrebbero essere di intralcio nell'interpretazione dei risultati ottenuti.

Nel disegno relativo al terzo esperimento le curve si dipartono dalle stazioni di lancio tratteggiate, poiché lampio intervallo di tempo tra lancio e ricupero da adito a maggiori incertezze sul percorso seguito dai galleggianti. 


\section{PR I MO LA NCIO}

\begin{tabular}{|c|c|c|c|c|c|}
\hline Rolta & $\begin{array}{c}\text { Posizione geografica della } \\
\text { stazione di lancio }\end{array}$ & Giorno & Ora & $\begin{array}{c}\text { Numero } \\
\text { delle schede }\end{array}$ & $\begin{array}{l}\text { Nave e Comandante } \\
\text { da cui fu eseguito il lancio }\end{array}$ \\
\hline Cagliari-Palermo & $\begin{array}{ll}\text { 甲 } & 38^{\prime \prime} \cdot 10^{\prime} 30^{\prime \prime} \mathrm{N} . \\
\text { i. } & 11^{\prime \prime} 18^{\prime} 30^{\prime \prime} \mathrm{E} . \mathrm{G} .\end{array}$ & 30 Gennaio 1952 & 01.00 & $101-150$ & $\begin{array}{l}\text { P/fo a Ichnusa " } \\
\quad(\text { Cap. R. Rinaudo) }\end{array}$ \\
\hline Napoli-Cargliari & $\begin{array}{l}\text { (f) } 3905318^{\prime \prime N} . \\
\text { i. } 11^{\prime \prime} 13^{\prime} 18^{\prime \prime} \text { E.G. }\end{array}$ & 29 Genniio 1952 & 20.30 & $51-100$ & $\begin{array}{l}\text { M/ve "Olbia " } \\
\text { (Cap. A. Csepely) }\end{array}$ \\
\hline Civitavecchia-Olbia & $\begin{array}{l}\text { I }+10300^{\prime}\left(00^{\prime \prime} \text { N. }\right. \\
\text { \% } 10^{\prime \prime}+1^{\prime}\left(00^{\prime \prime} \text { E.G. }\right.\end{array}$ & 30 Gennaio 1952 & 00.07 & $1-50$ & $\begin{array}{l}\text { M/ve "Cilli di Alessandr." } \\
\quad \text { (Cap. V. Sepich) }\end{array}$ \\
\hline Livorno-Bastia & 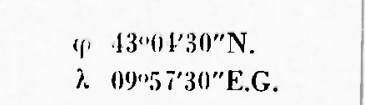 & $2 \%$ Gennaio 1952 & 03.20 & $151-200$ & $\begin{array}{l}\text { M/ve "Cittì di Livorno " } \\
\text { (Cap. M. Napoli) }\end{array}$ \\
\hline Genova-Porto Torres & $\begin{array}{ll}\text { "f } & 13^{\prime} 12^{\prime} 000^{\prime \prime} \text { N. } \\
\lambda & 08^{\prime \prime}+16^{\prime}\left(00^{\prime \prime} \text { E.G. }\right.\end{array}$ & 29 Gennaio 1952 & 15.35 & $201-250$ & $\begin{array}{l}\text { J'fo «Civitavecchia ) } \\
\text { (Cap. E. Malusa) }\end{array}$ \\
\hline
\end{tabular}


Primo lancio sulla rotta Cagrliari-Palermo effettuato dal P/fo "Ichnusa " in $\varphi 38^{\prime \prime} 40^{\prime} 30^{\prime \prime} \mathrm{N} ., \lambda 11^{\circ} 18^{\prime} 30^{\prime \prime}$ E.G., il 30 gennain 1952 alle ore 01.00 con vento da E. Corza 1 e mare poco mosso.

\section{R I TROVAMENT I}

\begin{tabular}{|c|c|c|c|c|c|c|c|c|}
\hline 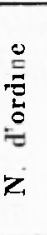 & 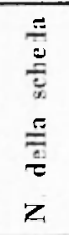 & $\mathrm{L} O \mathrm{O} C \mathrm{~A} \quad \mathrm{I}, \mathrm{I} \quad \mathrm{T} \boldsymbol{A}$ & $\begin{array}{c}\text { Posizione } \\
\text { geografica } \\
\text { approssimata }\end{array}$ & Giorno & Ora & 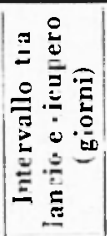 & Annotazioni & $\begin{array}{c}\text { Cognome di chi } \\
\text { ritrovò } \\
\text { il galleggiante }\end{array}$ \\
\hline 1 & 138 & Ad 1 miglio circa da Terasini-Palermo & $\begin{array}{l}38 \cdot 09^{\circ} \mathrm{N} \\
13 \cdot 05 \mathrm{E}\end{array}$ & 19.II.1952 & 10.00 & 20 & & G. Cracchiolo \\
\hline 2 & 112 & Spiaggia di Filicudi Gibbia-Messina & $\begin{array}{l}38^{\prime} 32^{\prime} \mathrm{N} \\
1+4^{\prime} 35^{\prime} \mathrm{i}\end{array}$ & $19-11 \cdot 1952$ & 16.00 & 20 & & M. Zagani \\
\hline 3 & 111 & Spiaggia di Castellamare del Golfo-Trapani & $\begin{array}{l}38^{\prime} \cdot\left(12^{\prime} \mathrm{N} \text {. }\right. \\
12^{\circ} 5^{\prime} \mathrm{F} \text {. }\end{array}$ & 21-I1-1952 & 12.00 & 22 & & G. Lal Rocca \\
\hline 4 & 139 & Spiaggia di Castellamare del Golfo-Trapani & ) & " & 16.30 & $》$ & & M. Valente \\
\hline 5 & 119 & Spiaggia di Castellamare del Golfo-Trapani & $m$ & $n$ & & 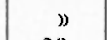 & & V. Navarra \\
\hline 6 & 124 & Spiaggia di Balestrate-Palermo & $\begin{array}{l}38^{\circ} 03^{\prime} \mathrm{N} . \\
13^{\circ}\left(00^{\prime} \mathrm{E} .\right.\end{array}$ & 22-II-1952 & 07.00 & 23 & & G. Galante \\
\hline 7 & 131 & Supiaggia di Balestrate-Palermo & $n$ & ” & ) & " & & $n$ \\
\hline 8 & 1.18 & Spiagnia di Balestrate-Palermo & $n$ & 》 & $n$ & $n$ & & $n$ \\
\hline 9 & 1.5 & Spiaggia di Aleamo Marina-Trapani & $\begin{array}{l}38^{\circ}\left(12^{*} \mathrm{~N}\right. \\
12057^{*} \mathrm{E}\end{array}$ & $23-11 \cdot 1952$ & 09.00 & 2.4 & & V. Maltese \\
\hline 10 & 149 & Spiaggia di Castellamare del Golfo-Trapuni & $\begin{array}{l}38^{\circ}(02 ' \mathrm{~N} \text {. } \\
12^{\circ} 53^{\prime} \mathrm{E} .\end{array}$ & $29-11.1952$ & 11.10 & 30 & & F. Navarra \\
\hline 11 & 141 & Spiagtia di Balestrate-Palermo & 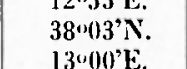 & $15-$ III-1952 & 12.00 & 45 & & G. Galinnte \\
\hline 12 & 110 & $\begin{array}{l}\text { Spliarian di Torre Faro (Località Calsa Bianca)- } \\
\text { Messina }\end{array}$ & $\begin{array}{l}38017 ' \mathrm{~N} . \\
15035 \mathrm{~K} .\end{array}$ & 25-III-1952 & 07.00 & 55 & & P. Mizzeo \\
\hline 13 & 113 & $\begin{array}{l}\text { A } 50 \text { m. circal, in direzione S.E., dall'Jsola Zan- } \\
\text { non:-lattina }\end{array}$ & 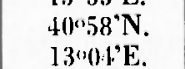 & l.V.1952 & 07.00 & 92 & & A. Di Santo \\
\hline 14 & 128 & A $\underset{\text { Messina }}{2}$ miglia cirea dalla costa del Golfo Tindaro- & $\begin{array}{l}38^{\circ} 10^{\prime} \mathrm{N} \\
15^{\circ}(05) \mathrm{E}\end{array}$ & $10 \cdot V \cdot I 952$ & 17.00 & 101 & & G. Nania \\
\hline
\end{tabular}




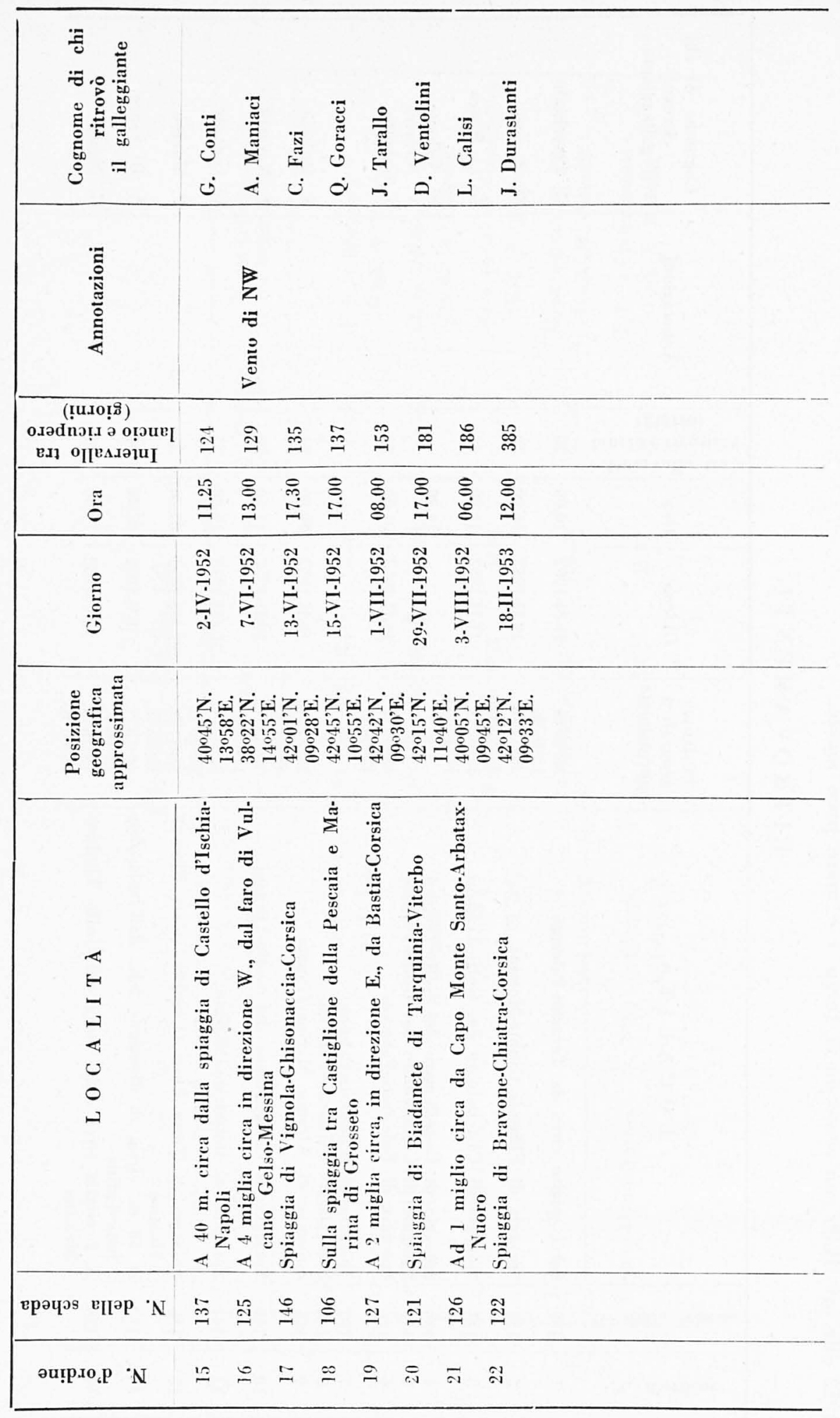


Primo lancio sulla rotta Napoli-Cagliari effettuato dalla M/ve "Citta di Livorno " in "f $39^{\prime \prime} 53^{\prime} 18^{\prime \prime}$ N., $i .11^{\circ} 43^{\prime} 48^{\prime \prime}$ E.G., il 29 gennaio 1952 , alle ore 20.30 con hava leggera di vento da $W N W$ e mare calmo.

\section{R I T R O V A M E N T I}

\begin{tabular}{|c|c|c|c|c|c|c|c|c|}
\hline 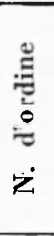 & 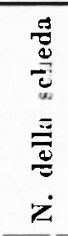 & 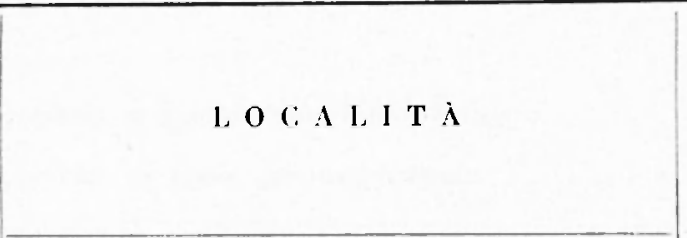 & $\begin{array}{c}\text { Posizione } \\
\text { geografical } \\
\text { approssimatia }\end{array}$ & Giorno & Ora & 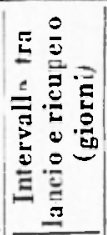 & Annotizioni & $\begin{array}{c}\text { Cognone di chi } \\
\text { ritrovó } \\
\text { il galleggiante }\end{array}$ \\
\hline 1 & ] 00 & $\begin{array}{l}\text { A miglia circa, in direzione NW, da Punta } \\
\text { Perciato-Isola Salina-Messina }\end{array}$ & $\begin{array}{l}38^{\prime \prime}: 19^{\prime} \mathrm{N} \\
14032 ' \mathrm{E} \text {. }\end{array}$ & $25-11-1952$ & 12.25 & 27 & & O. Tirinto \\
\hline 2 & 8.4 & $\begin{array}{l}\text { Ad } 1 \text { km. circa dalla spiaggia di Mongiove-Patti- } \\
\text { Messina }\end{array}$ & $\begin{array}{l}38^{\circ} 10^{\prime} \mathrm{N} . \\
1.1^{\circ} 58^{\prime} \mathrm{E} .\end{array}$ & $11-111-1952$ & 07.00 & 12 & & A. Paratore \\
\hline 3 & 60 & Spiaggia di Arquedolci-Messina & $\begin{array}{l}38^{\circ} 04 ' \mathrm{~N} \text {. } \\
1.1035^{\prime} \mathrm{E} .\end{array}$ & $18.111-1952$ & 14.00 & 49 & $\begin{array}{l}\text { Ritrovati solamente } \\
\text { lat cartolinat }\end{array}$ & F.A. Sritfidi \\
\hline 4 & 80 & $\begin{array}{l}\text { A } 3 \text { miglia circa, in direzione } \mathrm{NW} \text {, da Marina di } \\
\text { Caronia-Dlessina }\end{array}$ & $\begin{array}{l}38004 ' \mathrm{~N} . \\
14{ }^{\prime \prime 24} \mathrm{E} .\end{array}$ & $2.1-111-1952$ & 15.00 & 55 & & G. Urso \\
\hline 5 & 6.5 & $\begin{array}{l}\text { A } 20 \mathrm{~m} \text {. circal dall'imbocratura del porto di Capri- } \\
\text { Napoli }\end{array}$ & $\begin{array}{l}40^{\circ} 33^{\prime} \mathrm{N} . \\
14^{\circ} 15 \cdot \mathrm{E} .\end{array}$ & $15.1 V \cdot-1952$ & 09.30 & 77 & & G. Ruggiero \\
\hline 6 & 38 & $\begin{array}{l}\text { A lon m. cirra dalla spiaggia dei Maronti-S. An- } \\
\text { gelo-Isola d'Ischia-Nipoli }\end{array}$ & $\begin{array}{l}40^{\circ} 42^{\prime} \mathrm{N} \text {. } \\
13^{\circ} 5 .+\mathrm{E} \text {. }\end{array}$ & 22.IV.1952 & 17.00 & 8.1 & & $\begin{array}{l}\text { A. Iacono } \\
\text { F. Iacono }\end{array}$ \\
\hline 7 & 58 & Spiagria di Arquamorta-Honte di Procida-Napoli & $\begin{array}{l}40^{\circ} 18^{\prime} \mathrm{N} . \\
1+\cdots 2{ }^{\prime} \mathrm{E} .\end{array}$ & $25 . \mathrm{IV} \cdot 1952$ & 06.00 & 87 & & V. Pugliese \\
\hline 8 & 70 & A $10 \mathrm{~m}$. circa dalla spiaggia di Coroglio-Napoli & $\begin{array}{l}10^{\circ} 48^{\prime} \mathrm{N} . \\
1+11^{\prime} \mathrm{E} .\end{array}$ & $25 . I V \cdot 1952$ & 16.30 & 87 & & D. Ferrara \\
\hline 9 & 87 & $\begin{array}{l}\text { A } 23 \mathrm{~m} \text {. cirra dalla rada di Acquamorta-Monte } \\
\text { di Procida-Napoli }\end{array}$ & $\begin{array}{l}40^{\circ} 48^{\prime} \mathrm{N} . \\
14^{\circ}\left(12^{\prime} \mathrm{E}\right.\end{array}$ & 26-IV.1952 & 09.27 & 88 & & E. Moriseo \\
\hline 10 & 56 & Spiaggia di Lacona-Isola dThlba-livorno & $\begin{array}{l}42^{\circ} 46^{\prime} \mathrm{N} . \\
10^{\circ} 18^{\prime} \mathrm{F} .\end{array}$ & $6-\sqrt[V]{-1952}$ & 11.00 & 98 & & W. Sonchini \\
\hline 11 & 57 & Spiaggia di Laceo Ameno-Isola d'Ischia-Napoli & $\begin{array}{l}40 \cdot 15 ' \mathrm{~N} . \\
13^{\prime}, 53 \mathrm{k} .\end{array}$ & $15 \cdot \mathrm{V} \cdot 1952$ & 05.00 & 107 & & (i. Dorio \\
\hline 12 & 61 & Spiaggia di Chiarone (Stazione FF SS)-Grosseto & $\begin{array}{l}12 \cdot 23 ' N \text {. } \\
11027 ' \mathrm{E} \text {. }\end{array}$ & 13-VI-1952 & 07.00 & 136 & & C. Loli \\
\hline
\end{tabular}




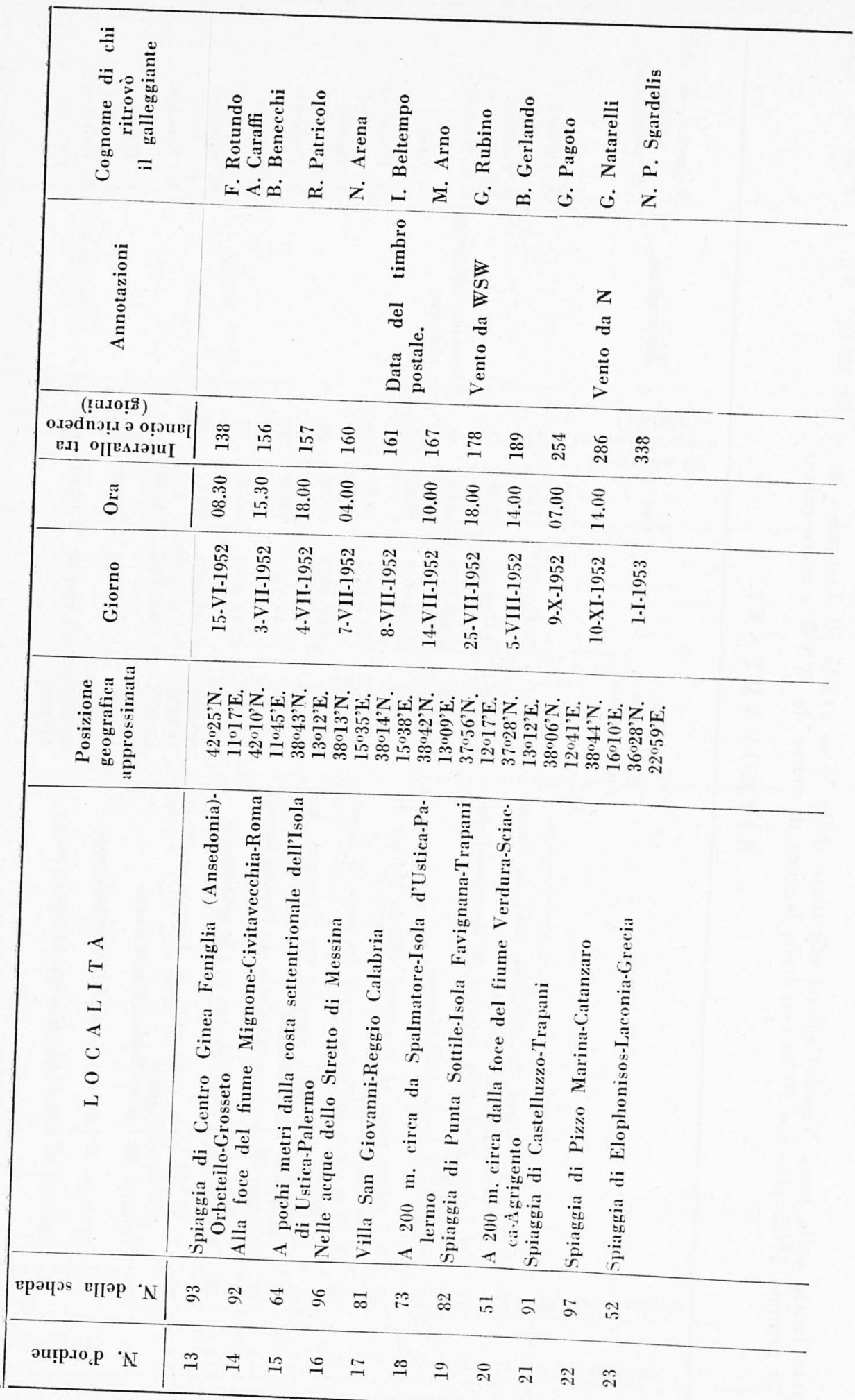


Primo lancio sulla rotta Civitavecchia-Ollia effettuato dalla M/ve "Ciltà di Alessandria " in $\varphi 41^{\circ} 30^{\prime} 00^{\prime \prime} \mathrm{N}$., $\lambda 10^{\circ} 41^{\prime} 00^{\prime \prime}$ E.G., il 30 gennaio 1952, alle ore 00.07 con brezza tesa da SW e mare legrermente mosso da SW.

\section{R I T R OV A M E N T I}

\begin{tabular}{|c|c|c|c|c|c|c|c|c|}
\hline 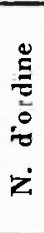 & 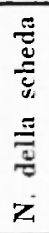 & $L \circlearrowleft C A L I T \dot{A}$ & $\begin{array}{c}\text { Posizione } \\
\text { geografica } \\
\text { approssimata }\end{array}$ & Giorno & Ora & 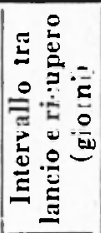 & Annotazioni & $\begin{array}{c}\text { Cognome di chi } \\
\text { ritrovò } \\
\text { il galleggiante }\end{array}$ \\
\hline 1 & 17 & Spiaggia di Rosignano Solvay-Iivorno & $\begin{array}{l}43^{\circ} 23^{\prime} \mathrm{N} . \\
10^{\circ} 26^{\prime} \mathrm{E} .\end{array}$ & $27 \cdot 111.1952$ & 06.30 & 57 & & M. Grandi \\
\hline 2 & 43 & Spiaggia di Viareggio (Zona Marco Polo)-Lucea & $\begin{array}{l}43^{\circ} 52^{\prime} \mathrm{N} \text {. } \\
10^{\circ} 15 ' \mathrm{E} \text {. }\end{array}$ & 27.111 .1952 & 14.30 & 57 & & C. Temporini \\
\hline 3 & 9 & Spiaggia di S. Raphael-Var.Francia & $\begin{array}{l}43 \circ 25^{\prime} \mathrm{N} \\
06^{\circ} \cdot 46^{\prime} \mathrm{E}\end{array}$ & $1 . I V \cdot 1952$ & & 62 & Bottiglia rotta & R. Pascal \\
\hline 4 & 45 & Sulla spiaggia tra Marina di l’isa e Tirrenia-Pisa & $\begin{array}{l}430.40 ' N . \\
10016 \cdot \mathrm{E} .\end{array}$ & $2 . I V \cdot 1952$ & 09.00 & 63 & & E. Rindi \\
\hline 5 & 40 & Spiaggia di S. Bartolomeo-La Spezia & $\begin{array}{l}44006 ' \mathrm{~N} . \\
09052^{\prime} \mathrm{E} .\end{array}$ & $2 . I V \cdot 1952$ & 13.00 & 63 & & A. Trincali \\
\hline 6 & 4 & $\begin{array}{l}\text { A } 15 \text { m. circa da Punta Libeccio-Pianosa Isola- } \\
\text { Livorno }\end{array}$ & $\begin{array}{l}42^{\circ} 35^{\prime} \mathrm{N} \\
10^{\circ} 03^{\circ} \mathrm{E}\end{array}$ & $6-1 V \cdot 1952$ & 21.00 & 67 & & R. Murtas \\
\hline 7 & 41 & Spiaggia di Bocca del Serchio-Pisa & $\begin{array}{l}43^{\circ} 47^{\prime} \mathrm{N} \\
10^{\circ} 16^{\prime} \mathrm{E} .\end{array}$ & $8-I V .1952$ & 09.00 & 69 & & o. Nori \\
\hline 8 & 14 & Spiaggia delle Rocee-Isola del Ievant-Var-Francia & $\begin{array}{l}43^{\circ} 01^{\prime} \mathrm{N} \\
06^{\circ} 28^{\prime} \mathrm{E}\end{array}$ & 9.IV.]952 & & 70 & & D. Relier \\
\hline 9 & 49 & $\begin{array}{l}\text { Nella darsena del Vallo di Porto S. Stefano-Gros- } \\
\text { seto }\end{array}$ & $\begin{array}{l}42^{\circ} 26{ }^{\prime} N \text {. } \\
11^{\circ} 077^{\prime} \mathrm{E} .\end{array}$ & $12-1 \mathrm{~V} \cdot 1952$ & 07.00 & 73 & & D. Fanciulli \\
\hline 10 & 5 & $\begin{array}{l}\text { Sulla spiaggia tra Agde e Séte (a } 2 \mathrm{~km} \text {. da Séte). } \\
\text { Hérault-Francia }\end{array}$ & $\begin{array}{l}43^{\circ} 23^{\prime} \mathrm{N} . \\
03^{\circ} 40^{\prime} \mathrm{E} .\end{array}$ & 17.IV.1952 & & 78 & & J. Camuzat \\
\hline 11 & 2 & $\begin{array}{l}\text { A } 150 \text { m. circa da Capo Caldo-Golfo di Porto Az- } \\
\text { zurro-Isola d'Elba-Livorno }\end{array}$ & $\begin{array}{l}420.44 \mathrm{~N} \text {. } \\
10026, \mathrm{E} .\end{array}$ & 24-1V-1952 & 16.00 & 85 & & A. Chicea \\
\hline 12 & 3 & Spiaggia di Ja Londe Les Maures-Var-Francia & $\begin{array}{l}43^{\circ} 07 ' N \text {. } \\
06015^{\prime} \mathrm{E} .\end{array}$ & $2 \cdot V \cdot 1952$ & 09.00 & 93 & & N. N. \\
\hline
\end{tabular}




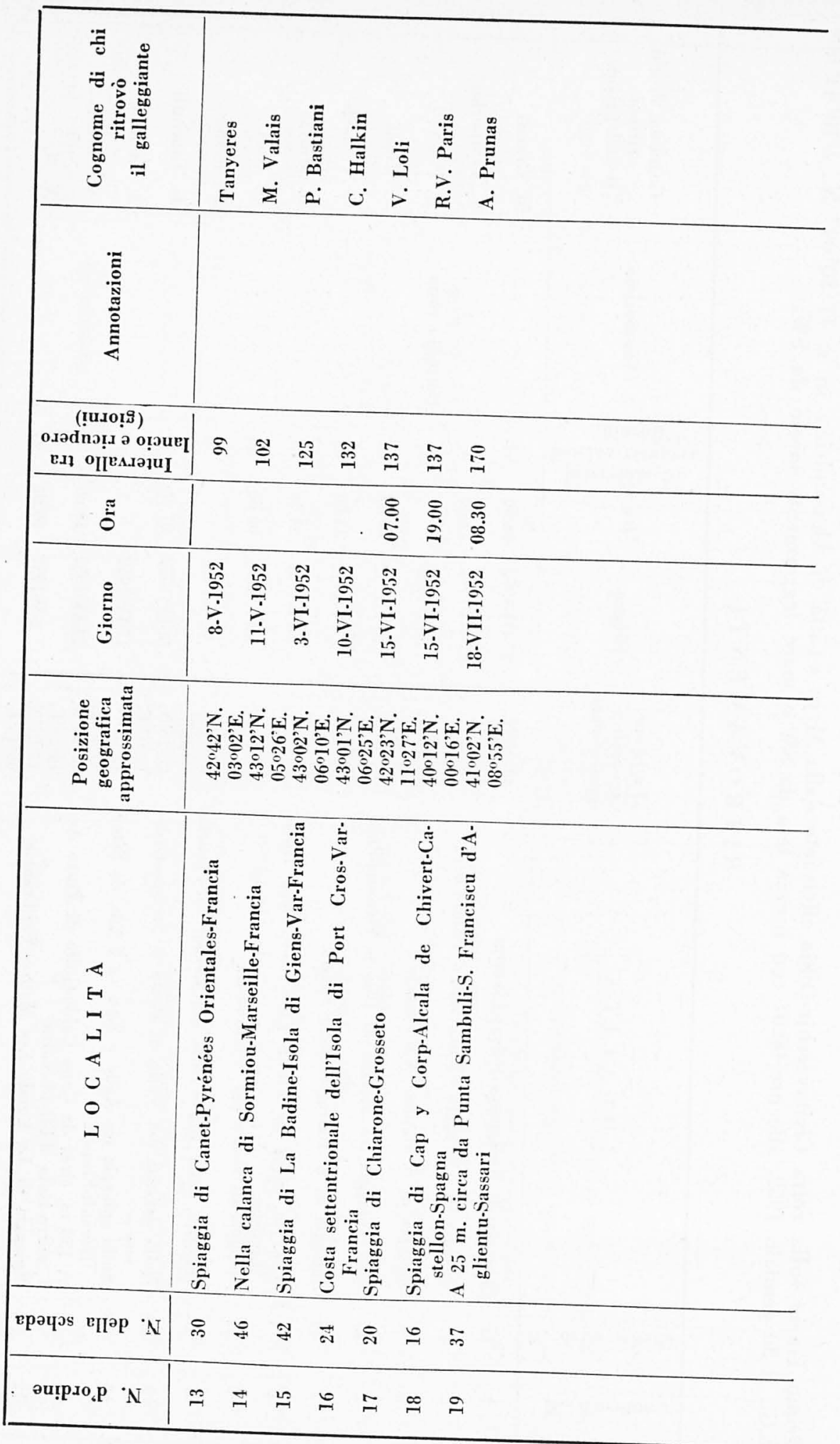




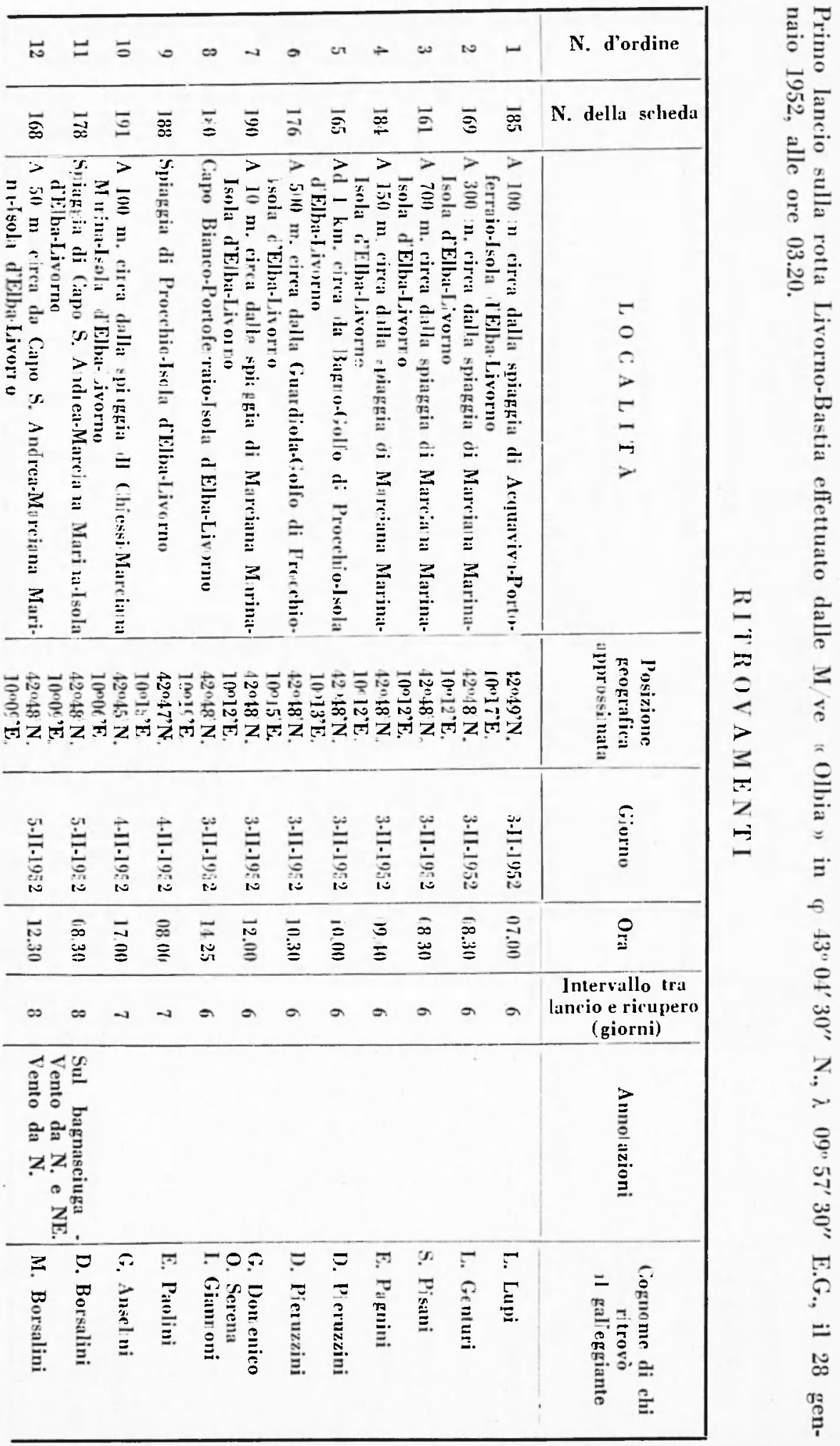




\begin{tabular}{|c|c|c|c|c|c|c|c|c|c|c|c|c|c|c|}
\hline 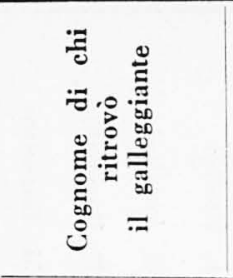 & 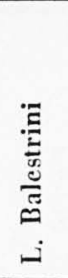 & $\begin{array}{l}\ddot{\Xi} \\
\text { ఏ } \\
\dot{3}\end{array}$ & $\underset{\Sigma}{\Sigma}$ & $\begin{array}{l}\ddot{\Xi} \\
\ddot{\Xi} \\
\stackrel{\Xi}{\Xi} \\
\dot{\Sigma}\end{array}$ & 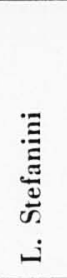 & 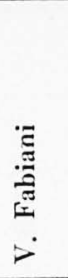 & $\begin{array}{l}\Xi \\
\Xi \\
\dot{0}\end{array}$ & $\begin{array}{l}\ddot{\Xi} \\
\dot{e} \\
\dot{e} \\
\dot{0} \\
\dot{0}\end{array}$ & $\begin{array}{l}\vec{\Xi} \\
\stackrel{\Xi}{0} \\
ن\end{array}$ & 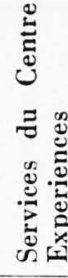 & 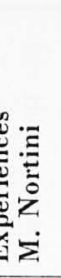 & $\begin{array}{l}ت \tilde{\Xi} \\
\text { के } \\
ن\end{array}$ & 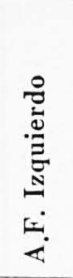 & $\begin{array}{l}\frac{0}{0} \\
.00 \\
0 \\
\vdots \\
0 \\
0\end{array}$ \\
\hline 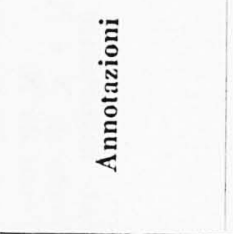 & & & & & & & & & 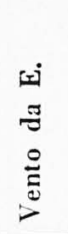 & & & & & \\
\hline 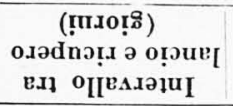 & $\infty$ & $\infty$ & $\infty$ & $a$ & $\stackrel{2}{\sim}$ & 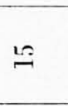 & $\bar{ন}$ & ন & 은 & $\because$ & $\ddot{\infty}$ & $\tilde{a}$ & \& & 兽 \\
\hline 5 & $\underset{\Xi}{\Xi}$ & 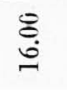 & 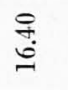 & $\stackrel{8}{\varrho}$ & $\begin{array}{l}\varrho \\
g\end{array}$ & $\stackrel{\varrho}{\varrho}$ & 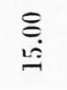 & $\underset{\varrho}{\varrho}$ & $\stackrel{12}{2}$ & & & $\begin{array}{l}\varrho \\
\varrho\end{array}$ & & $\underset{\Xi}{\Xi}$ \\
\hline$\stackrel{\mathscr{E}}{\mathscr{E}}$ & $\frac{\sqrt{2}}{\stackrel{2}{\Xi}}$ & 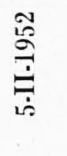 & 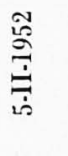 & 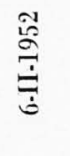 & 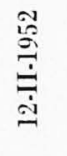 & 䄊 & 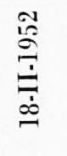 & 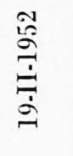 & 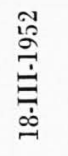 & 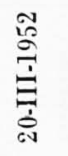 & 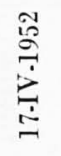 & 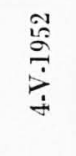 & $\frac{\pi}{\stackrel{2}{2}}$ & 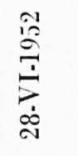 \\
\hline 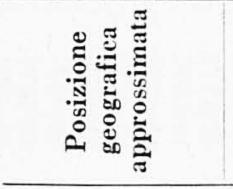 & \multicolumn{14}{|c|}{ 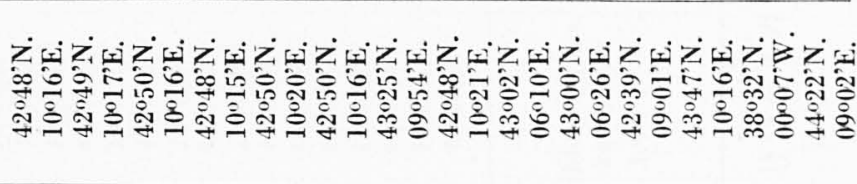 } \\
\hline $\begin{array}{l}4 \\
5 \\
-1 \\
-1 \\
4 \\
0 \\
0 \\
-1\end{array}$ & 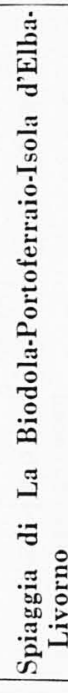 & 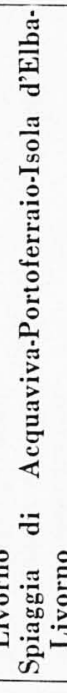 & 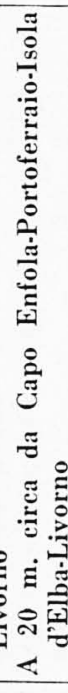 & 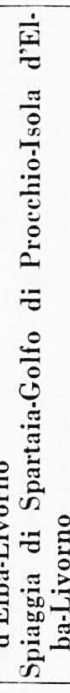 & 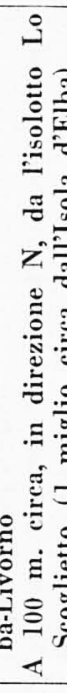 & 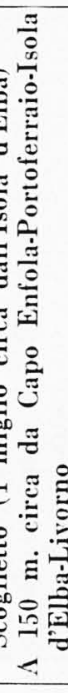 & 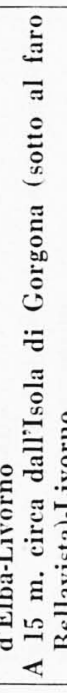 & 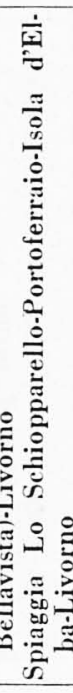 & 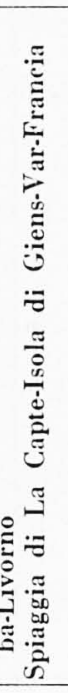 & 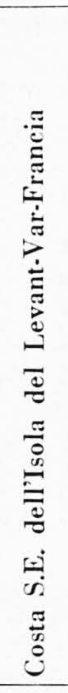 & 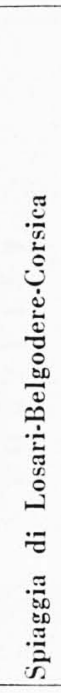 & 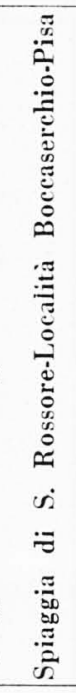 & 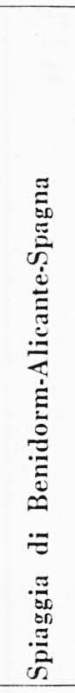 & 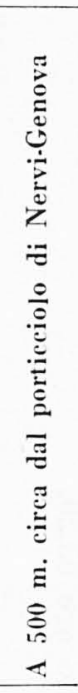 \\
\hline ерәчәs е & $\stackrel{\Re}{=}$ & 낙 & $\stackrel{+}{0}$ & $\stackrel{\infty}{\infty}$ & $\stackrel{\overbrace{}}{\stackrel{(}{N}}$ & $\vec{\Xi}$ & $\stackrel{\infty}{\infty}$ & $\ddot{\theta}$ & 总 & $\stackrel{\Im}{\sigma}$ & $\stackrel{\infty}{\stackrel{2}{\curvearrowleft}}$ & $\stackrel{9}{2}$ & 冢 & 兽 \\
\hline 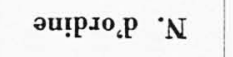 & $\ddot{n}$ & 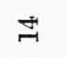 & 20 & $\because$ & $\simeq$ & $\stackrel{\infty}{\sim}$ & 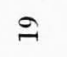 & $\approx$ & $\overline{\text { }}$ & ป & $\approx$ & ম্ & 농 & $\stackrel{\sim}{\sim}$ \\
\hline
\end{tabular}




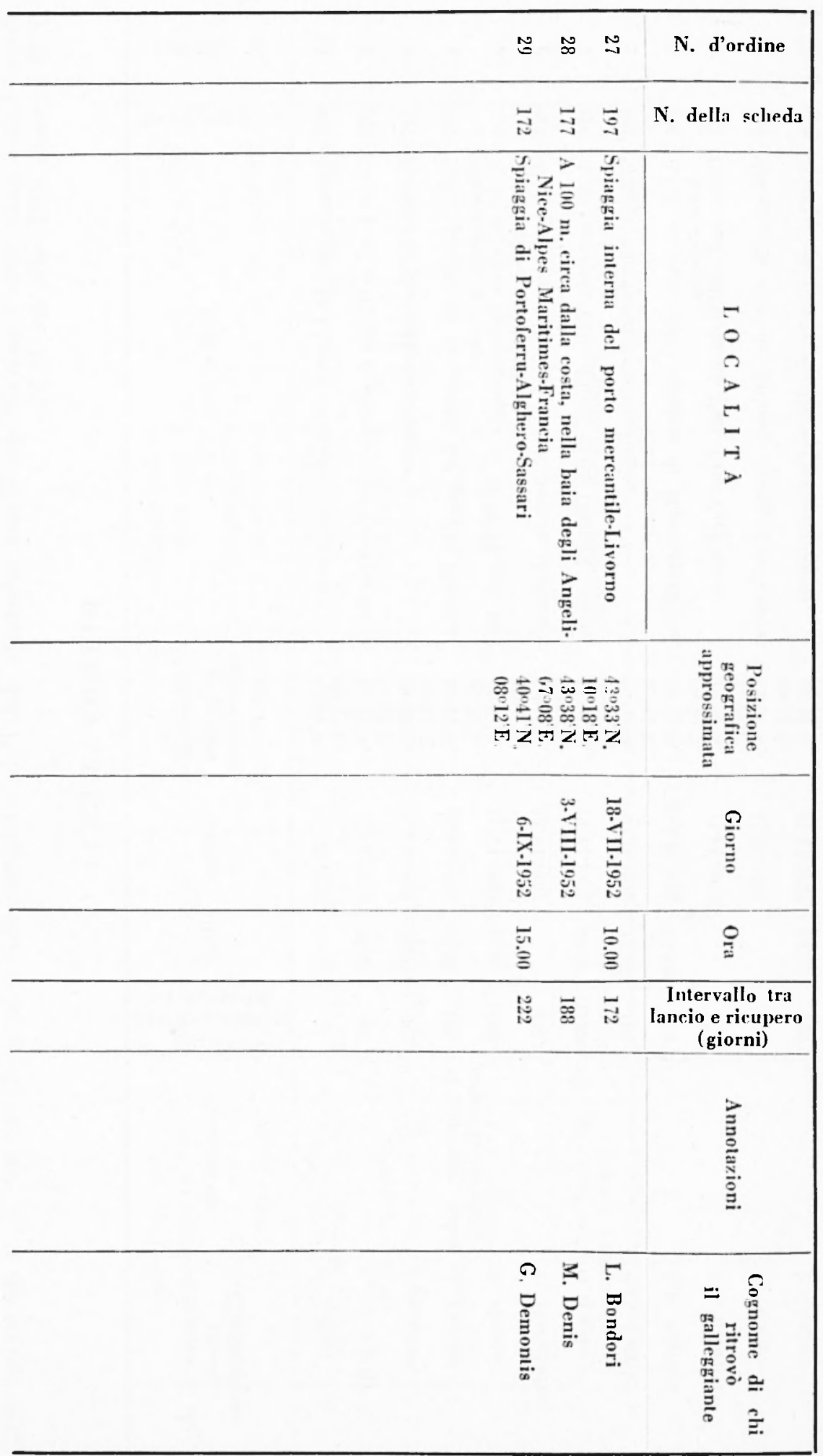


Primo lancio sulla rotta Genova-Porto Torres effettuato dal P/fo "Civitavecchia " in $\varphi \quad 43^{\circ} 42^{\prime} 00^{\prime \prime} N . \lambda^{\prime} \lambda 8^{\circ} 46^{\prime} 00^{\prime \prime}$ E.G., il 29 gennaio 1952, alle ore 15.35.

\section{RI'TOVAMENTI}

\begin{tabular}{|c|c|c|c|c|c|c|c|c|}
\hline 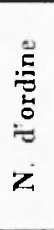 & 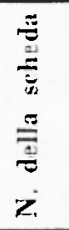 & L O C A L I T A & $\begin{array}{c}\text { Posizione } \\
\text { geogrifica } \\
\text { approssimatta }\end{array}$ & Giomo & Or: & 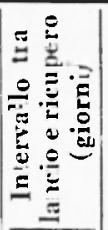 & Annotizioni & $\begin{array}{l}\text { Cognome di clì } \\
\text { ritrovò } \\
\text { il galleggiante }\end{array}$ \\
\hline 1 & 205 & Spiategia di Cap Cépet-S. Mandriér-Var-Francia & $\begin{array}{l}43 \cdot 0+4 . N . \\
05,57 \mathrm{E} .\end{array}$ & 20.111 .1952 & & 51 & \multirow{12}{*}{$\begin{array}{l}\text { Si rinvenne unica- } \\
\text { mente la cartolina. }\end{array}$} & P. Jolivet \\
\hline 2 & 207 & Costil nord dellisola di Porquerolles-Var-Franeja & $\begin{array}{l}43^{\prime \prime}(1)^{\prime} \mathbf{N} \text {. } \\
\text { (1)6"13'E. }\end{array}$ & $6.1 \mathrm{~V} \cdot 19.52$ & 17.00 & 68 & & Ecole T.E.R. \\
\hline 3 & 2.45 & Spiaggia di Javea-Alininte-Spagna & $\begin{array}{l}38^{\prime}+8^{\prime} \mathrm{N} \text {. } \\
0\left(0^{\prime} 11^{\prime} \mathrm{F} .\right.\end{array}$ & $22 . I V \cdot 1952$ & $06.0 \mathrm{i})$ & 84 & & J.G. Vives \\
\hline 4 & 225 & $\begin{array}{l}\text { Sulla spiaggia in prossimitit del pontile Petrolea } \\
\text { tria Savonal e Valdo }\end{array}$ & $\begin{array}{l}\text { H'"17'N. } \\
\text { (i8'28'E. }\end{array}$ & $1.5 . V 1.1952$ & 06.00 & 138 & & (;. Fiorito \\
\hline 5 & 210 & $\begin{array}{l}\text { A } 20 \mathrm{~m} \text {. eircal dalla spiaggia di Mola (Bagni Mi- } \\
\text { ramare)-Varazze-Savonal }\end{array}$ & 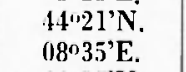 & 17.51 .1952 & 12.00 & 140 & & D. Giusto \\
\hline 6 & 228 & A t m. circa dalla spiaggia di Savona (Fornaci) & $\begin{array}{l}44^{\circ} 17^{\prime} \mathrm{N} \\
08^{\circ} 28^{\prime} \mathrm{E} \text {. }\end{array}$ & $22 . \mathrm{VI} \cdot 1952$ & & 145 & & I). Durighello \\
\hline 7 & 226 & $\begin{array}{l}\text { A } 30 \text { m. circa dalla Punta della Civitata-Caleraia } \\
\text { Isola-Livorno }\end{array}$ & $\begin{array}{l}43^{\circ}\left(0 l^{\prime} \mathrm{N} .\right. \\
09^{\circ} 5 \mathrm{l}^{\prime} \mathrm{E} .\end{array}$ & $29 \cdot V 1.1952$ & 10.20 & 152 & & G. Lupi \\
\hline 8 & 209 & Spiaggia di laraco.Valencialspagna & $\begin{array}{l}39^{\circ} 03^{\prime} \mathrm{N} \text {. } \\
00^{\circ} 11^{N} \mathrm{~W} \text {. }\end{array}$ & 9.VII-1952 & 11.00 & 162 & & A.G. Carrillo \\
\hline 9 & 2.16 & $\begin{array}{l}\text { A } 30 \text { m. rircal dalla spiaggia di Masamagrell-Yal } \\
\text { lenciar-Spannal }\end{array}$ & $\begin{array}{l}39^{\circ} 35 \% \text {. } \\
00^{4} 16^{\prime} W\end{array}$ & $20 . \mathrm{VIJ} \cdot 1952$ & 18.00 & 173 & & B.F. Soriano \\
\hline 10 & 2.1 .1 & Costa sud dell'isola di Port Cros-Var-Francia & $\begin{array}{l}43^{\circ} 00^{\prime} \mathrm{N} \\
06^{n} 2 \cdot 4^{\prime} \mathrm{E}\end{array}$ & $1.1 X \cdot 1952$ & & 216 & & J.C. Lebeltel \\
\hline 11 & 250 & Spliaggia di Cap de Salins-S. Trope\%.Var-Francia & $\begin{array}{l}13^{\circ} 07^{\prime} N \text {. } \\
06^{\circ} 12^{\prime} \mathrm{E} \text {. }\end{array}$ & $17.1 X \cdot 1952$ & & 232 & & L. Walter \\
\hline 12 & 224 & Spriaggia di Cocria di Morto-Fiumicino-Roma & $\begin{array}{l}41^{\circ}+18^{\prime} \mathrm{N} \\
12^{\circ} 13^{\prime} \mathrm{E}\end{array}$ & $12 \cdot X I \cdot 1952$ & 10.13 & 288 & & L. Pagano \\
\hline
\end{tabular}


SECONDO LA NCIO

\begin{tabular}{|c|c|c|c|c|c|}
\hline Rotta & $\begin{array}{l}\text { Posizione geografica della } \\
\text { stazione di lancio }\end{array}$ & Giormo & Ora & $\begin{array}{c}\text { Numero } \\
\text { delle schede }\end{array}$ & $\begin{array}{l}\text { Nave e Comandante } \\
\text { da cui fu eseguito il lancio }\end{array}$ \\
\hline Cangliari-Palermo & $\begin{array}{ll}\uparrow & 38^{\circ} 38^{\prime} 00^{\prime \prime} \mathrm{N} \\
\lambda & 11^{\circ} 17^{\prime} 00^{\prime \prime} \mathrm{E} . \mathrm{G} .\end{array}$ & 26 Marzo 1952 & 01.10 & $391-460$ & $\begin{array}{l}\text { P/fo “Ichnusa » } \\
\quad(\text { Cap. E. Colozzo) }\end{array}$ \\
\hline Napoli-Cagliari & $\begin{array}{ll}\text { if } 39^{\circ} 53^{\prime} 30^{\prime \prime} \mathrm{N} . \\
\lambda .11^{\circ} 45^{\prime}\left(00^{\prime \prime} \mathrm{E} . \mathrm{G} .\right.\end{array}$ & 25 Marzo 1952 & 21.06 & $321-390$ & $\begin{array}{l}\text { M/ve "Città di Livorno " } \\
\text { (C.ap. M. Napoli) }\end{array}$ \\
\hline Civitavecchia-Olbia & $\begin{array}{l}\text { if } 41^{\circ} 31^{\prime} 00^{\prime \prime} \mathrm{N} . \\
\text { i. } 10^{\circ} 40^{\prime} 00^{\prime \prime} \mathrm{E} . \mathrm{G} .\end{array}$ & 26 Marzo 1952 & 00.45 & $251 \cdot 320$ & $\begin{array}{l}\text { M/ve (Citti di Trapani ») } \\
\text { (C.ip. A. Del Pino) }\end{array}$ \\
\hline Livorno-Bastia & $\begin{array}{ll}\varphi & 43^{\circ} 055^{\prime} 00^{\prime \prime} \mathrm{N} . \\
\lambda & 09^{\circ} 58^{\prime} 00^{\prime \prime} \mathrm{E} . \mathrm{G} .\end{array}$ & 23 Marzo 1952 & 03.37 & 461.530 & $\begin{array}{l}\text { M/ve «Olbia » } \\
\text { (Cap. A. Csepely) }\end{array}$ \\
\hline Genova-Porto Torres & $\begin{array}{ll}\text { क } & 43^{\prime \prime} 43^{\prime} 00^{\prime \prime} \mathrm{N} . \\
\lambda & 00^{\circ} 14^{\prime} 00^{\prime \prime} \mathrm{E} . \mathrm{G} .\end{array}$ & 25 Marzo 1952 & 15.20 & $531 \cdot 600$ & $\begin{array}{l}\text { P/fo "Civitavecchia » } \\
\text { (Ciap. M. Poggi) }\end{array}$ \\
\hline
\end{tabular}




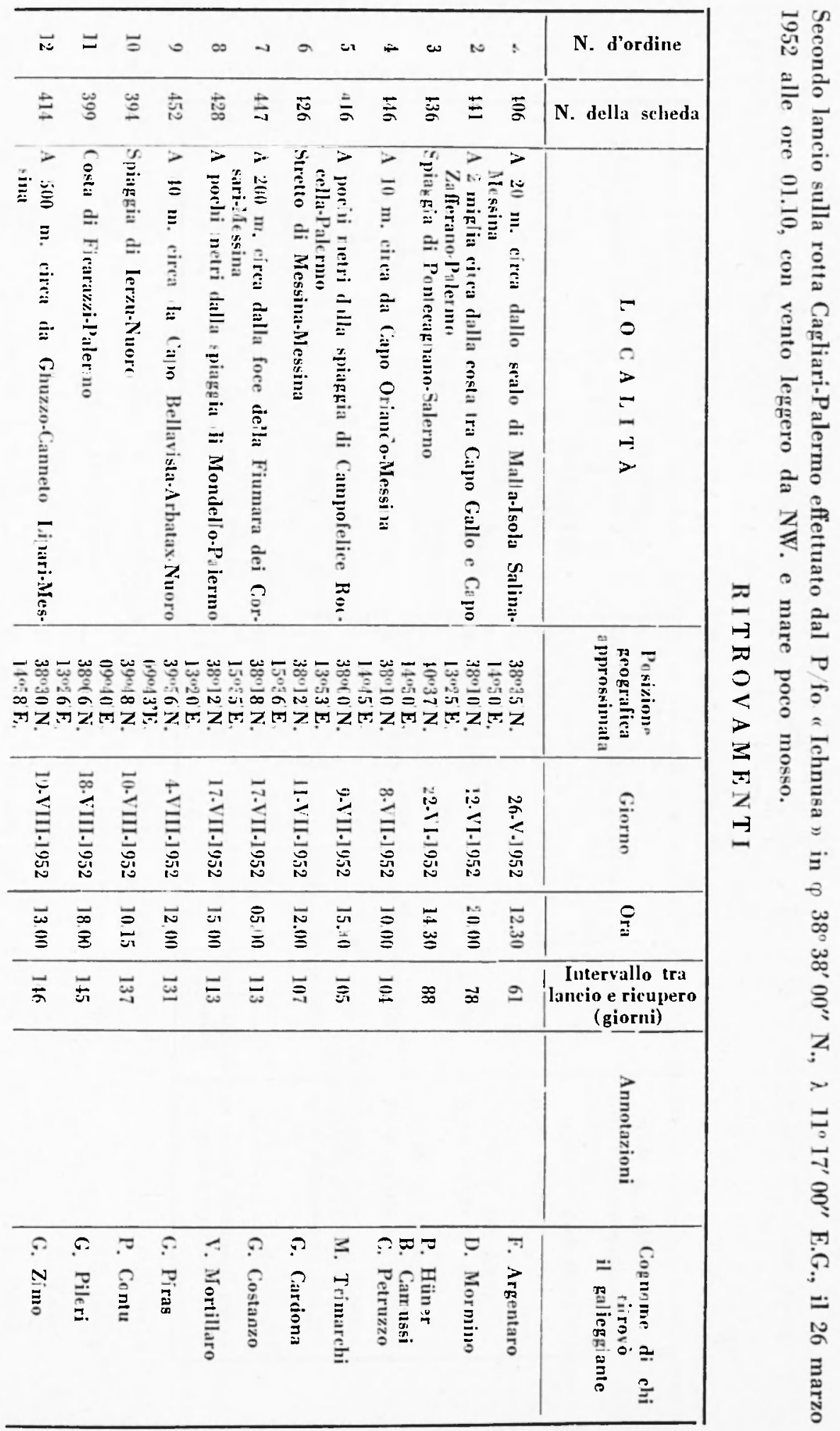




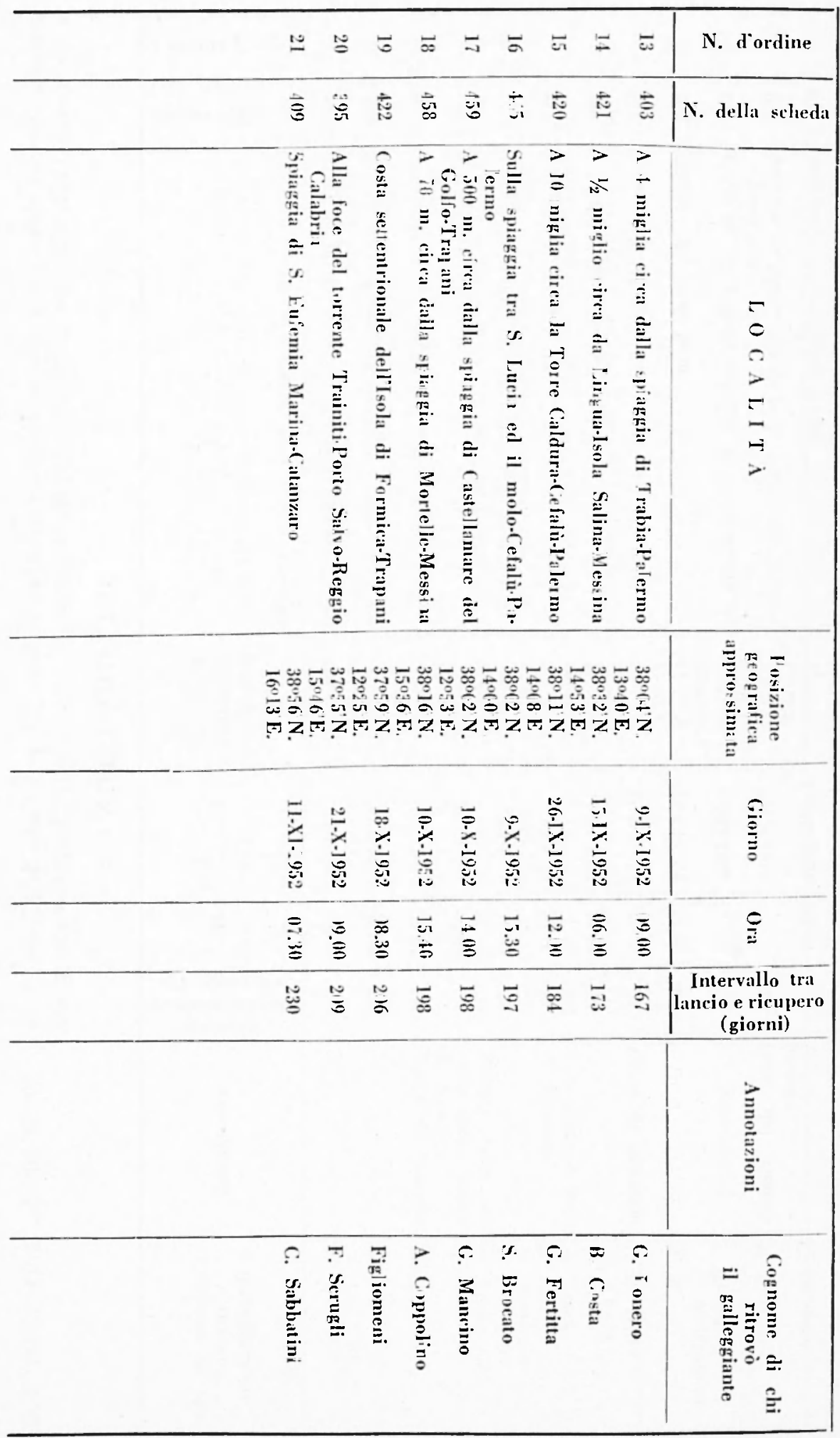


Secondo lancio sulla rotla Napoli-Canliari effelluato dalla M/ve "Citti di Livorno " in $\varphi \quad 39^{\circ} 53^{\prime} 30^{\prime \prime} \mathrm{N}$., $\lambda 1^{\circ} 45^{\prime} 00^{\prime \prime}$ E.G., il 25 marzo 1952, alle ore 21.06, con hrezza leggera da SW. e mare leggermente mosso.

\section{R I T R OV A M EN T I}

\begin{tabular}{|c|c|c|c|c|c|c|c|c|}
\hline 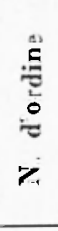 & 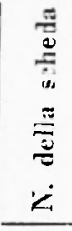 & I. $O C A \mathrm{~L}, \mathrm{I} T \dot{\mathrm{A}}$ & $\begin{array}{c}\text { Posizione } \\
\text { geografica } \\
\text { approssimata }\end{array}$ & Ginrno & Ora & 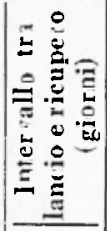 & Annotazioni & $\begin{array}{c}\text { Cognome di chi } \\
\text { ritrovò } \\
\text { il galleggiante }\end{array}$ \\
\hline 1 & 339 & Spiaggia di Punta dei Mastixi-Barisardo-Nuoro & $\begin{array}{l}39 \cdot 500^{\prime} \mathrm{N} . \\
00^{\circ} 42^{\circ} \mathrm{E} .\end{array}$ & $3.1 \cdot 1952$ & 23.00 & 39 & & V. Storhino \\
\hline 2 & 333 & Golfo di Porto Vecelio.Corsica & $\begin{array}{l}41 \cdot 36 \mathrm{~N} . \\
09 \cdot 20 ' \mathrm{E} .\end{array}$ & $19 \cdot V \cdot 1952$ & 15.30 & 55 & & Forroni \\
\hline 3 & 331 & $\begin{array}{l}\text { A } 30 \text { m. circal dalla costa nel Golfo di Orosei- } \\
\text { Nuoro }\end{array}$ & $\begin{array}{l}40 \cdot 15^{\prime} \mathrm{N} . \\
09038 \mathrm{E} .\end{array}$ & $28 \cdot V \cdot 1952$ & 14.00 & 6.1 & & M. Pala \\
\hline 4 & $3 \pi . i$ & Spiaggia Poetto-Cagliari & $\begin{array}{l}390111^{\prime} \mathrm{N} . \\
\left(090100^{\prime} \mathrm{E} .\right.\end{array}$ & $29 \cdot V \cdot 1952$ & 06.40 & 6.5 & & R. Mancia \\
\hline 5 & 350 & $\begin{array}{l}\text { Spiaggia di Nlbinia } 12 \mathrm{hm} \text {. a } \mathrm{N} \text {. dellabitato)-Grose } \\
\text { seto }\end{array}$ & $\begin{array}{l}42 \cdot 31 ' N . \\
11 \cdot 11 ' E .\end{array}$ & $9 . V I \cdot 1952$ & 18.00 & 76 & & A. Smaniotto \\
\hline 6 & 337 & Spiaggia di Aleano Marina-Trapani & $\begin{array}{l}38 \cdot() 1, N \text {. } \\
12 \cdot 56 \cdot \mathrm{E} .\end{array}$ & $9 . Y 1.1952$ & 20.15 & 76 & & G. Plaia \\
\hline$i$ & 389 & Cala daecqua-Ie Forna-Iatina & $\begin{array}{l}40,55 \times \mathrm{N} \text {. } \\
12.58 \mathrm{E} .\end{array}$ & 1].VI.1952 & 22.30 & 78 & & A. Cristo \\
\hline 8 & 381 & Spiaggia di Albarese.Crosseto & $\begin{array}{l}1238 \mathrm{~N} \text {. } \\
11{ }^{\prime}\left(04^{\prime} \mathrm{E}\right.\end{array}$ & $16 . V 1.1952$ & 09.00 & 83 & Vento di Scirocco. & V. Di Mitto \\
\hline 9 & 386 & Spiaggia di Anzio-Roma & $\begin{array}{l}11 \cdot 26 \mathrm{~N} . \\
12 \cdot 37 \mathrm{E} .\end{array}$ & $29 . \mathrm{VI} .1952$ & 18.00 & 96 & & N. N. \\
\hline 10 & 321 & $\begin{array}{c}\text { A } 300 \mathrm{~m} . \text { cirea dalla spiaggia di Osalla.Golfo di } \\
\text { Orosei-Nuoro }\end{array}$ & $\begin{array}{l}40^{\prime \prime} 19^{*} \mathrm{~N} \\
0 g^{\prime \prime}+2^{\prime} \mathrm{F} .\end{array}$ & 4-VII.1952 & 17.00 & 101 & & S. Romino \\
\hline 11 & 363 & Spiaggia di Punta sottile-Isola Favignana-Trapani & $\begin{array}{l}37 \cdot 56^{\prime} \mathrm{N} . \\
12 \cdot 16^{\prime} \mathrm{E} .\end{array}$ & 7.111 .19 .52 & & 10.4 & $\begin{array}{l}\text { Data lel timbro } \\
\text { postale. }\end{array}$ & M. Torrente \\
\hline 12 & 373 & Spiaggia di Civitaverechia-Roma & $\begin{array}{l}12 "() 6 ’ \mathrm{~N} \\
11^{\prime \prime} 48^{\prime} \mathrm{E} .\end{array}$ & $10 . V 11.1952$ & 11.35 & 107 & & E. Iacopuni \\
\hline
\end{tabular}




\begin{tabular}{|c|c|c|c|c|c|c|c|c|c|}
\hline 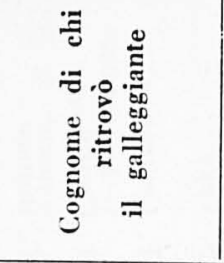 & $\begin{array}{l}\stackrel{\mathscr{N}}{\mathbb{N}} \\
\dot{\tilde{z}} \\
\dot{\Sigma}\end{array}$ & 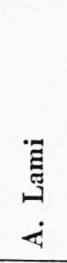 & 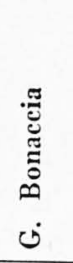 & 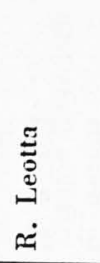 & 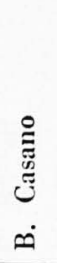 & $\begin{array}{l}\ddot{\Xi} \\
\Xi \\
\dot{\Xi}\end{array}$ & 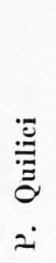 & 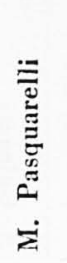 & 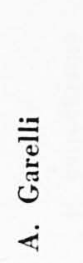 \\
\hline 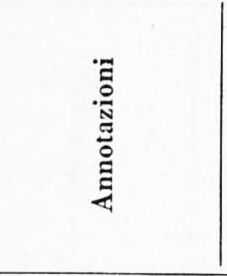 & \multicolumn{9}{|c|}{ 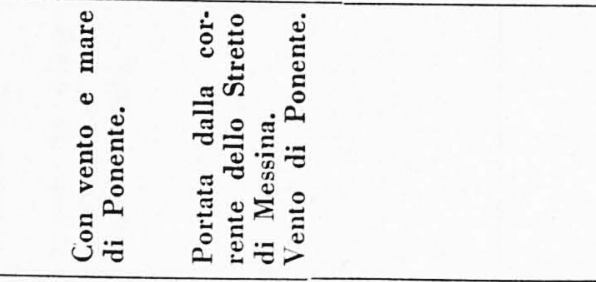 } \\
\hline 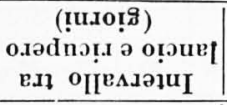 & $\stackrel{\infty}{=}$ & $\stackrel{\check{a}}{\sim}$ & $\stackrel{5}{\sigma}$ & $\stackrel{\mathscr{\sigma}}{=}$ & 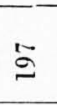 & $\bar{\Xi}$ & $\stackrel{\stackrel{0}{\ominus}}{\circ}$ & $\stackrel{0}{\sim}$ & 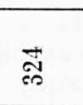 \\
\hline$\tilde{5}$ & $\underset{\vdots}{\infty}$ & $\stackrel{\cong}{\cong}$ & 눙 & $\stackrel{\Xi}{\Xi}$ & 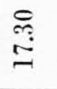 & $\underset{\sigma}{0}$ & & 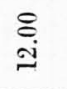 & $\underset{\Xi}{0}$ \\
\hline 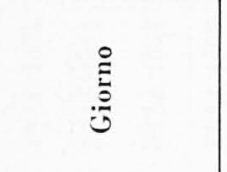 & 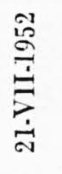 & $\frac{\text { Iै }}{2}$ & 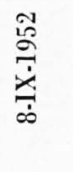 & 営 & 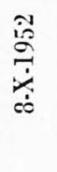 & 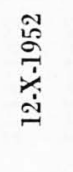 & 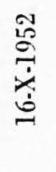 & 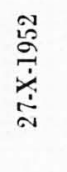 & 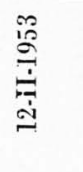 \\
\hline 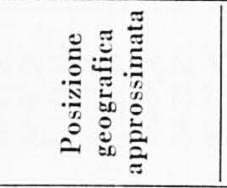 & \multicolumn{9}{|c|}{ 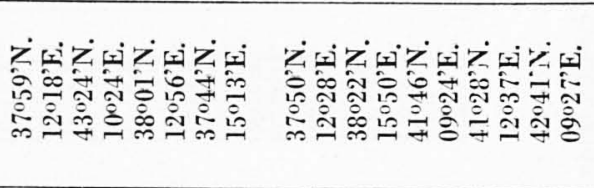 } \\
\hline $\begin{array}{l}4 \\
\text { H } \\
1 \\
1 \\
4 \\
0 \\
0 \\
0\end{array}$ & 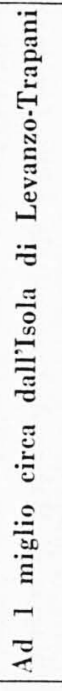 & 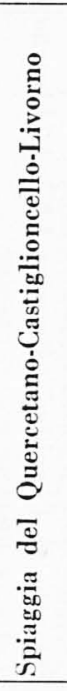 & 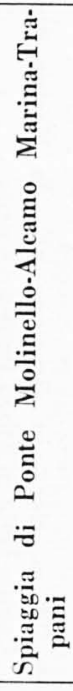 & 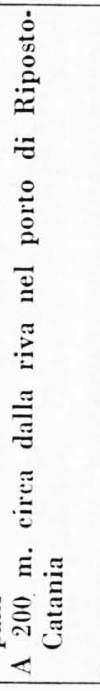 & 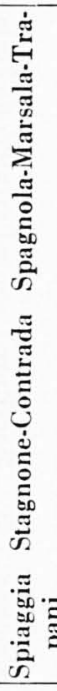 & 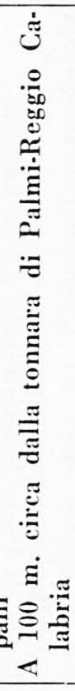 & 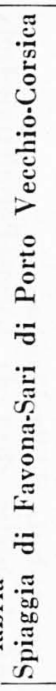 & 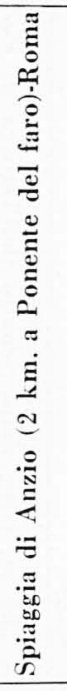 & 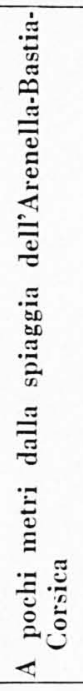 \\
\hline ерацәв $\mathbf{v}_{[I \partial p} \cdot \mathrm{N}$ & 屃 & 今ి & $\ddot{r}$ & 灾 & 总 & 占 & 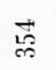 & 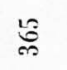 & ๙ัँ \\
\hline әu!p.so.p $\cdot \mathrm{N}$ & $\dddot{7}$ & $\ddot{\Xi}$ & $\stackrel{20}{7}$ & $\stackrel{\circ}{\sim}$ & $\simeq$ & $\cong$ & $\approx$ & ลิ & $\bar{ล}$ \\
\hline
\end{tabular}


Sccondo lancio sulla rolla Civitavecchia-Olbia effetluato dalla M/ve "Citla di Trapani " in ip $4 l^{\circ} 3 l^{\prime} 00^{\prime \prime} \mathrm{N}$., $\lambda 10^{\prime \prime} 40^{\prime} 30^{\prime \prime}$ E.G., il 26 marzo 1952, alle ore 00.45.

RITROVA M E N T I

\begin{tabular}{|c|c|c|c|c|c|c|c|c|}
\hline 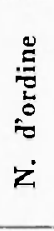 & 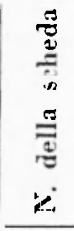 & I. O $： \Lambda \mathrm{L}, \mathrm{l} \mathrm{T} \wedge$ & $\begin{array}{c}\text { Posizione } \\
\text { geografica } \\
\text { alprossimata }\end{array}$ & Giorno & Ora & 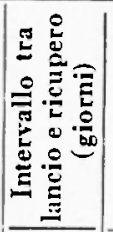 & Annotazioni & $\begin{array}{l}\text { Cognome di chi } \\
\text { ritrovò } \\
\text { il galleggiante }\end{array}$ \\
\hline 1 & 266 & $\begin{array}{l}\text { A } 200 \text { m. cirea dallo seoglio dellogliera di Po- } \\
\text { monte-lsola d Ellat-Livorno }\end{array}$ & $\begin{array}{l}42 " 45 \times N \\
10 \cdots 17 \mathrm{E} .\end{array}$ & $17 . I Y \cdot 1952$ & 11.30 & 22 & & A. Galearzi \\
\hline 2 & 296 & $\begin{array}{l}\text { Ad l miglio rimal da Lacona-Portoferraio-Isola } \\
\text { dêlba-Livorno }\end{array}$ & $\begin{array}{l}42 " 4+N \text {. } \\
10^{\prime \prime} 18 \cdot \mathrm{E} \text {. }\end{array}$ & $18-1 \mathrm{~V} \cdot 1952$ & 14.00 & 23 & & G. Muti \\
\hline 3 & 260 & Colfo di Porto Azzurro-Isola d'Ellbal-Livorno & $\begin{array}{l}42^{\prime}+46^{\prime} \mathrm{N} \text {. } \\
10 \cdot 2+\mathrm{E} \text {. }\end{array}$ & $21.1 \mathrm{~V} .19 .52$ & 18.00 & 26 & & C: Riffo \\
\hline 4 & $27 t$ & $\begin{array}{l}\text { Spiagdia di Nargitore-latona-Porloferraio-Isola } \\
\text { dFiba-Livorno }\end{array}$ & $\begin{array}{l}42^{\circ} 46^{\circ} \mathrm{N} \\
10^{\circ} 19^{\circ} \mathrm{E} .\end{array}$ & $22.1 \mathrm{~V} \cdot 1952$ & 08.00 & 27 & & L. Burelli \\
\hline 5 & .315 & Splatgtia ali Marina di Campo-Isola d'Elba-Livorno & $\begin{array}{l}42^{\circ}+5 ' N . \\
10^{\prime \prime} 14 \cdot \mathrm{E} .\end{array}$ & 22.IV-1952 & 10.00 & 27 & & M. Paolini \\
\hline 6 & 290 & $\begin{array}{l}\text { A } 500 \mathrm{~m} \text {. rirca, in direzione SF., da Marina di } \\
\text { (ampo-Isolat d'Elbal-Livorno }\end{array}$ & $\begin{array}{l}42^{\circ} 45^{\prime} \mathrm{N} . \\
10^{\prime \prime} 11^{\circ} \mathrm{E} .\end{array}$ & $22.1 \mathrm{~V} .1952$ & 10.30 & 27 & & E. Paolini \\
\hline 7 & 2.58 & Apiatepia di Piombino (discarica I.L.V.A.)-Livorno & $\begin{array}{l}42,55 ’ \mathrm{~N} \\
10^{\prime} 33^{\prime} \mathrm{E}\end{array}$ & 22-IV-1952 & 10.37 & 27 & & F. Barni \\
\hline 8 & 291 & Fpiagkial di Míarina di Campo-Isola d'Elba-Livorno & $\begin{array}{l}42^{\prime \prime} 45^{\prime} \mathrm{N} . \\
10^{\prime \prime} 14 \mathrm{E} \text {. }\end{array}$ & 22-IV-1952 & 11.00 & 27 & & M. Dini \\
\hline 9 & 257 & $\begin{array}{l}\text { Ad I hm. rirea dalla spiaggia Moreone-Capoliveri- } \\
\text { Isola d Elba-Idivorno }\end{array}$ & $\begin{array}{l}42 " 44 \mathrm{~N} \text {. } \\
10 \cdots 22 \mathrm{E} \text {. }\end{array}$ & $27-I V-1952$ & 11.00 & 32 & & S. Vago \\
\hline 10 & 276 & $\begin{array}{l}\text { A lo0 m. circa dalla spiaggia di Favona-Sari di } \\
\text { Porto Vecrho-Corsica }\end{array}$ & $\begin{array}{l}41^{\circ}+4 \mathrm{~N} \\
09 \cdot 24 \mathrm{E}\end{array}$ & 29-] V.1952 & 11.00 & 31 & & F. Centrune \\
\hline 11 & 318 & $\begin{array}{l}\text { A porhi metri dalla Punta di Marina di Campo } \\
\text { (f.olle)-Isola d'Elbar-Livorno }\end{array}$ & $\begin{array}{l}12 \cdots 14 N . \\
10014 \mathrm{E}\end{array}$ & $29 . I V .1952$ & 18.00 & 34 & & I. Paolini \\
\hline 12 & 261 & $\begin{array}{l}\text { Ad } 800 \mathrm{~m} \text {. rirca dalla spiaggia di S. Vincenzo-Ia- } \\
\text { vorno }\end{array}$ & $\begin{array}{l}13^{\circ} 06^{\circ} \mathrm{N} \\
10^{\circ} 31^{\circ} \mathrm{E} .\end{array}$ & 30.IV.1952 & 19.30 & 3.5 & & G. Butlaro \\
\hline
\end{tabular}




\begin{tabular}{|c|c|c|c|c|c|c|c|c|c|c|c|c|c|c|c|}
\hline 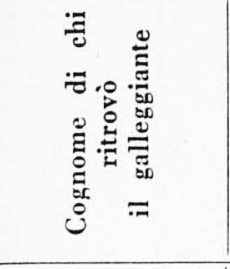 & 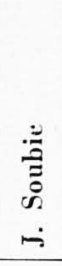 & $\begin{array}{l}Z \\
Z \\
Z\end{array}$ & 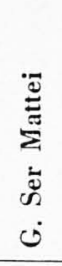 & 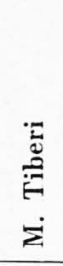 & 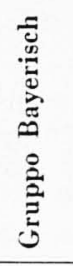 & 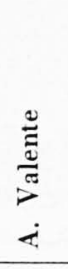 & 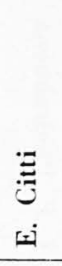 & 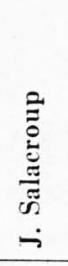 & 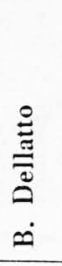 & $\begin{array}{l}\stackrel{\Xi}{\Xi} \\
\stackrel{\Xi}{\Xi} \\
\dot{D}\end{array}$ & 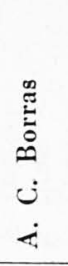 & $\begin{array}{l}\ddot{\Xi} \\
\dot{0} \\
\dot{a} \\
\dot{\Xi} \\
\dot{4}\end{array}$ & 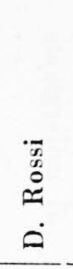 & $\begin{array}{l}\stackrel{\Xi}{ \pm} \\
\stackrel{\Xi}{\Xi} \\
\dot{4}\end{array}$ & 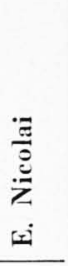 \\
\hline 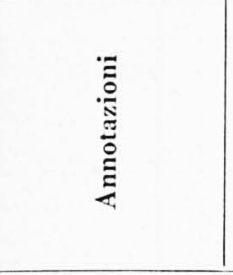 & 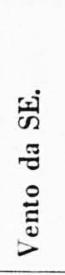 & 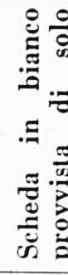 & 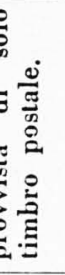 & & & & & & 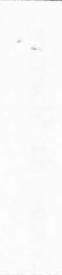 & & & & & & \\
\hline 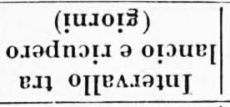 & $\tilde{s}$ & F & $\stackrel{0}{2}$ & 오ำ & $\overrightarrow{0}$ & $\vec{b}$ & $\approx$ & $\approx$ & $\stackrel{2}{ }$ & ᄃ) & $\therefore$ & $\stackrel{\infty}{=}$ & ஸे & 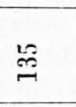 & تָ \\
\hline 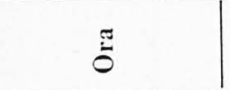 & $\underset{\Xi}{\stackrel{\Xi}{二}}$ & & $\stackrel{\text { ֻุ }}{=}$ & & $\underset{\Xi}{0}$ & $\underset{\Xi}{\Xi}$ & $\stackrel{\oplus}{\varrho}$ & & $\stackrel{\Xi}{=}$ & $\underset{0}{\varrho}$ & $\underset{\overbrace ָ}{\check{n}}$ & & $\underset{\vdots}{\stackrel{\Xi}{\Xi}}$ & $\stackrel{\Xi}{=}$ & 空 \\
\hline$\stackrel{0}{\vdots}$ & 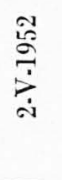 & $\frac{i}{\frac{i}{2}}$ & 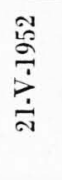 & 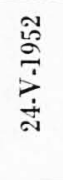 & 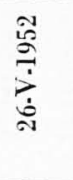 & 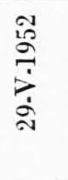 & $\frac{1}{\stackrel{2}{a}}$ & 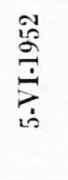 & 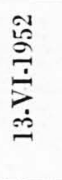 & 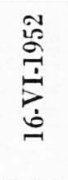 & 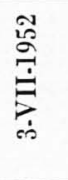 & 咅 & 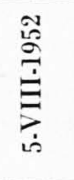 & $\underset{\infty}{\stackrel{i}{:}}$ & 总 \\
\hline 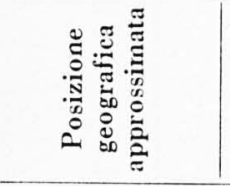 & \multicolumn{15}{|c|}{ 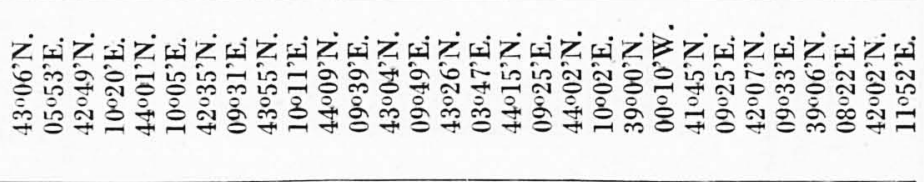 } \\
\hline $\begin{array}{l}4 \\
= \\
= \\
4 \\
0 \\
0 \\
=\end{array}$ & 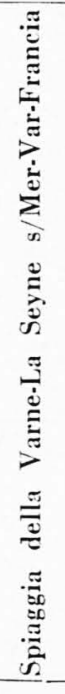 & 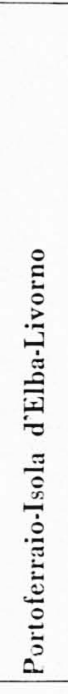 & 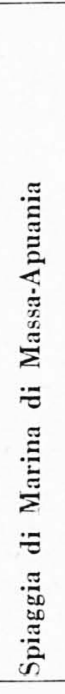 & 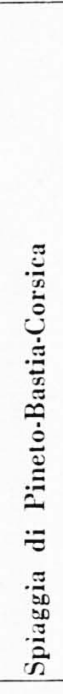 & 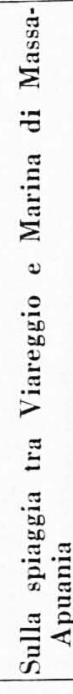 & 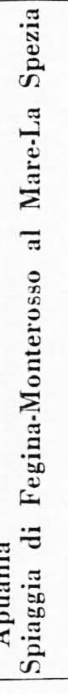 & 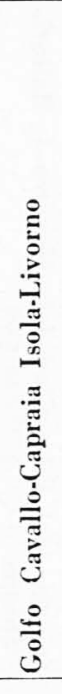 & 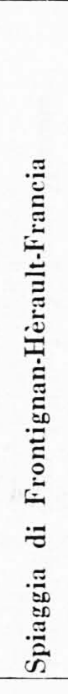 & 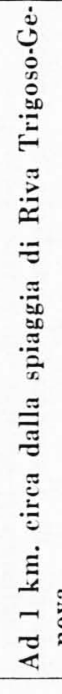 & 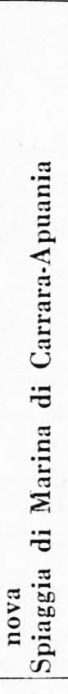 & 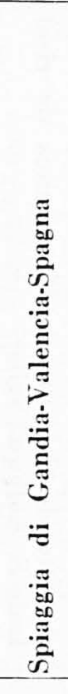 & 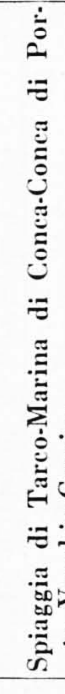 & 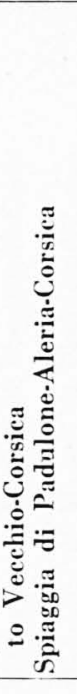 & 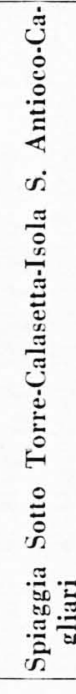 & 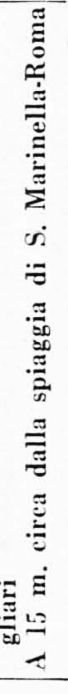 \\
\hline 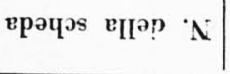 & $\stackrel{\curvearrowleft}{\sigma}$ & $\stackrel{\Re}{\circ}$ & $\vec{\sigma}$ & ๖ํ. & $\tilde{\sigma}$ & $\underset{\sim}{\stackrel{\sim}{*}}$ & ণ్లి & $\stackrel{\approx}{\pi}$ & $\vec{m}$ & $\stackrel{m}{\tilde{m}}$ & 怘 & $\vec{\sigma}$ & $\widetilde{\sigma}$ & $\underset{\sim}{\sim}$ & $\widetilde{\infty}$ \\
\hline әu!p.os $\quad \mathrm{N}$ & $\stackrel{9}{-1}$ & $\vec{ت}$ & $\stackrel{2}{\sim}$ & 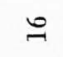 & $\approx$ & $\stackrel{\infty}{\oplus}$ & $\approx$ & ণ & ส & สี & ฆ & $\vec{A}$ & 농 & $\stackrel{\infty}{i}$ & $\check{\sim}$ \\
\hline
\end{tabular}




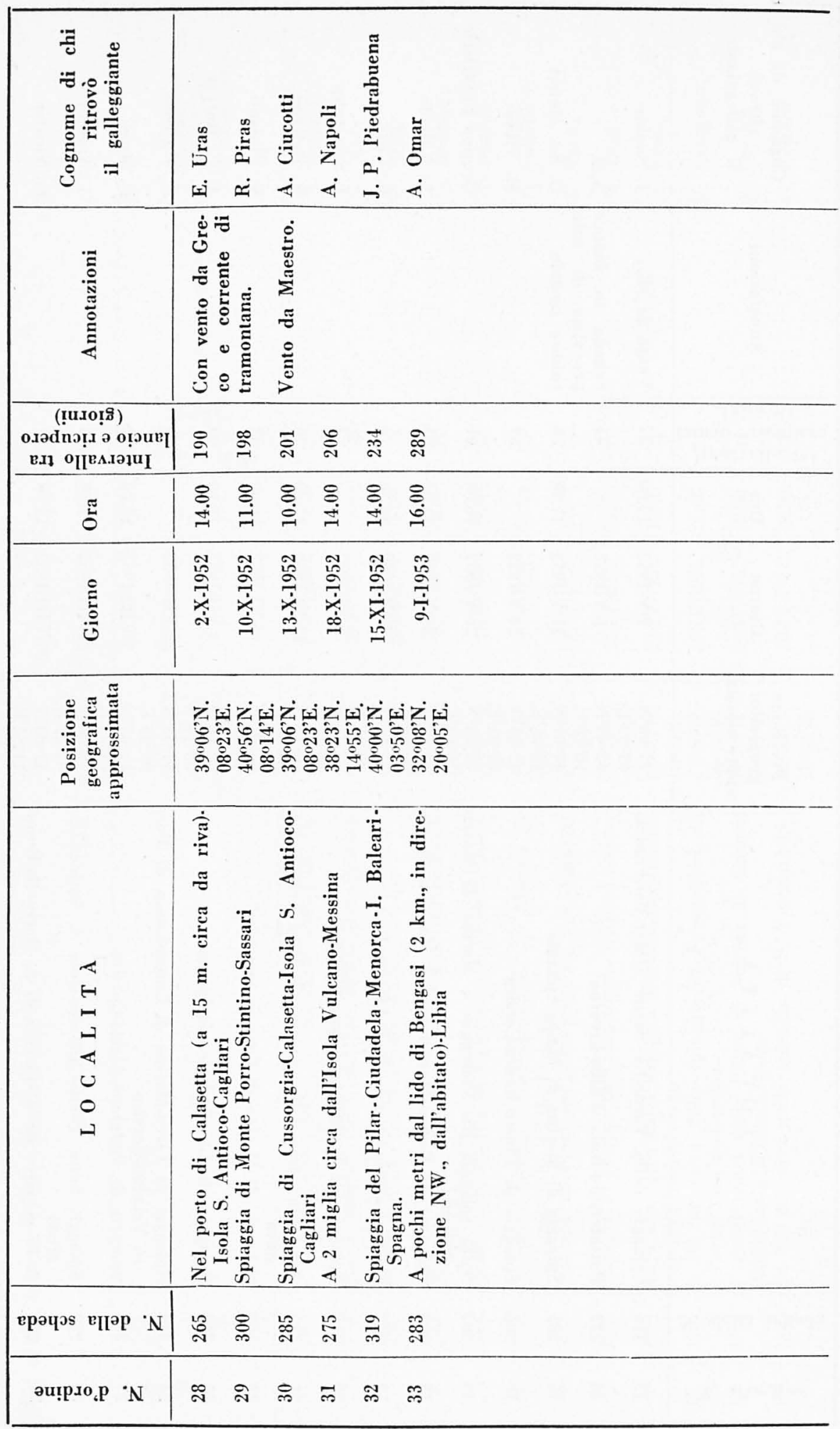




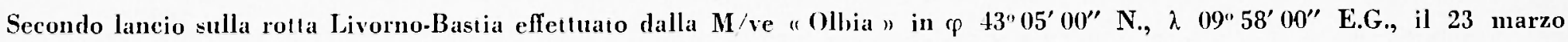
1952, alle ore 03.37 .

\section{R I'TROVA M EN T I}

\begin{tabular}{|c|c|c|c|c|c|c|c|c|}
\hline 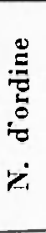 & 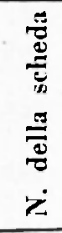 & L. $O C \Lambda$ L I I $\Lambda$ & $\begin{array}{c}\text { Posizione } \\
\text { geografica } \\
\text { approssimata }\end{array}$ & Giorno & Ora & 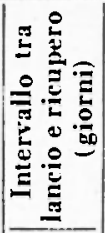 & Annotazioni & $\begin{array}{c}\text { Cognome di chi } \\
\text { ritrovó } \\
\text { il galleggiante }\end{array}$ \\
\hline 1 & 481 & Spiaggia Bagni Fiume-Livorno & $\begin{array}{l}13 \cdot 33^{\prime} \mathrm{N} . \\
10^{\prime \prime} 18^{\circ} \mathrm{E}\end{array}$ & 31.111 .1952 & 06.30 & 8 & & A. Gherardi \\
\hline 2 & 465 & Spiaggia di S. Rossore-Pisa & $\begin{array}{l}43^{\prime \prime 4}+3^{\prime} \mathrm{N} . \\
10^{\prime} \cdot 16^{\prime} \mathrm{E} .\end{array}$ & $31-111-1952$ & 16.00 & 3 & & o. Croci \\
\hline 3 & 530 & Spiaggia di Bocca d'Arno-Marina di Pisa-P'isa & $\begin{array}{l}43 \cdot 41 \% N . \\
10 \cdot 16 \mathrm{E} .\end{array}$ & 31.111 .1952 & 16.00 & i & & P. Bottai \\
\hline 4 & 497 & Spiaggia di S. Rossore-Pisa & $\begin{array}{l}43^{\prime}+3 \mathrm{~N} \\
10^{\prime} 16^{\circ} \mathrm{E}\end{array}$ & $31.111-1952$ & 18.00 & 8 & & P. Bottai \\
\hline 5 & 513 & Spiaggia Torre del Lago Purcini-Lucea & $\begin{array}{l}43^{\prime \prime 49} \mathrm{~N} \text {, } \\
10^{\prime \prime} 15 \cdot \mathrm{E} \text {. }\end{array}$ & $2 \cdot I V \cdot 1952$ & 06.00 & 10 & $\begin{array}{l}\text { Ricuperata con Li- } \\
\text { heccio. }\end{array}$ & A. Andreozzi \\
\hline 6 & 529 & Spiaggia della darsena di Viareggio-Luccal & $43.52 \mathrm{~N}$ & $2.1 V-1952$ & 06.30 & 10 & & S. Dattola \\
\hline 7 & 475 & Spiaggia di Marina di Pietrasanta-Lucca & $43.56 \mathrm{~N}$. & $2 \cdot I V \cdot 1952$ & 06.30 & 10 & & L. Bertocehi \\
\hline 8 & 520 & Spiaggia della darsena di Viareggio-Lucea & $43.52 \%$ & $2.1 V .1952$ & 07.00 & 10 & & A. I)attnla \\
\hline 9 & 515 & Spiaggia di Marina di Pietrasanta-Lucea & $\begin{array}{l}10^{\circ} 15^{\prime} \mathrm{E} . \\
43^{\prime \prime} 56^{\prime} \mathrm{N}\end{array}$ & $2.1 V .1952$ & 08.00 & 10) & & E. Corsi \\
\hline 10 & 528 & Spiaggia di Marina di Pietrasanta-Lucea & $\begin{array}{l}10 " 12 ' \mathrm{E} . \\
" y\end{array}$ & $"$ & 08.30 & 10 & . & $"$ \\
\hline 11 & 518 & Spiaggia di Marina di Pietrasanta-Lucea & $"$ & $"$ & $n$ & 10 & & ” \\
\hline 12 & 511 & Spiaggia di Marina di Pietrasanta-Lucca & ” & $"$ & " & 10 & & 》 \\
\hline
\end{tabular}




\begin{tabular}{|c|c|c|c|c|c|c|c|c|c|c|c|c|c|c|}
\hline 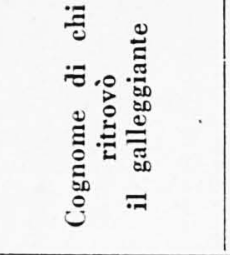 & 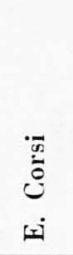 & $\approx$ & 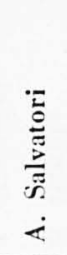 & 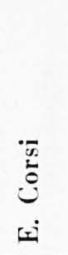 & 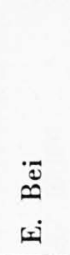 & 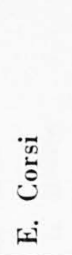 & 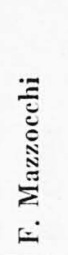 & 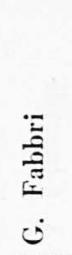 & $\underbrace{\infty}_{0}$ & $\approx$ & $\approx$ & 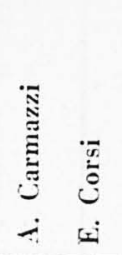 & 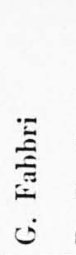 & 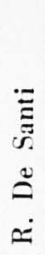 \\
\hline & & & & & & & & & & & & & & \\
\hline 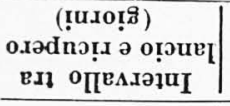 & 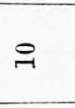 & 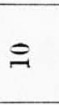 & $\cong$ & 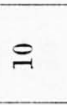 & 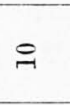 & $\cong$ & $\cong$ & $\cong$ & 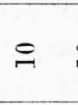 & $\cong$ & $\cong$ & $\cong$ & $\stackrel{\imath}{ }$ & $\cong$ \\
\hline 5 & @̊ & $\approx$ & $\stackrel{\Xi}{g}$ & $\stackrel{g}{\varrho}$ & $\stackrel{\Xi}{=}$ & $\stackrel{\Xi}{g}$ & 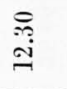 & 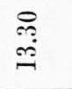 & $\underset{\Xi}{\Xi}$ & $\approx$ & $\approx$ & 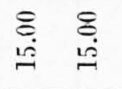 & $\sum_{1}$ & $\stackrel{\vdots}{\infty}$ \\
\hline$\stackrel{\varrho}{\vdots}$ & $\begin{array}{l}\frac{1}{2} \\
\stackrel{2}{2} \\
\dot{2}\end{array}$ & $\approx$ & $\frac{i}{i}$ & 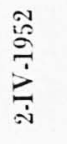 & 总 & 雍 & $\approx$ & $\frac{\dddot{2}}{2}$ & $\frac{3}{3}$ & $\approx$ & $\approx$ & 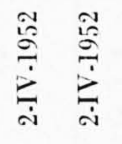 & $\frac{i}{2}$ & $=$ \\
\hline 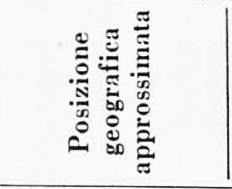 & \multicolumn{2}{|c|}{ 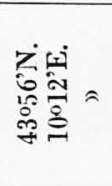 } & 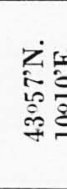 & 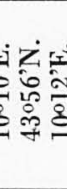 & 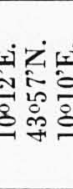 & 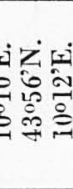 & $\frac{1}{1}$ & 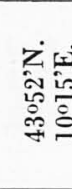 & 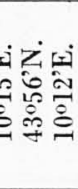 & $=$ & $\approx$ & \multicolumn{2}{|c|}{ 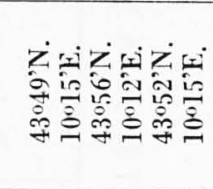 } & \\
\hline $\begin{array}{l}4 \\
5 \\
-1 \\
-1 \\
4 \\
0 \\
0 \\
0\end{array}$ & 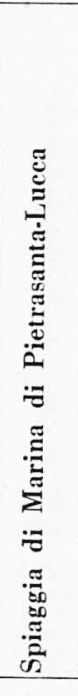 & 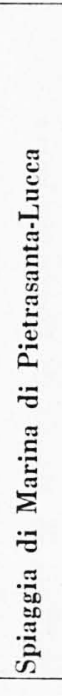 & 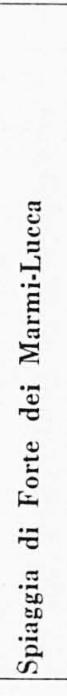 & 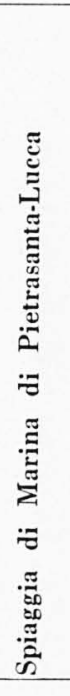 & 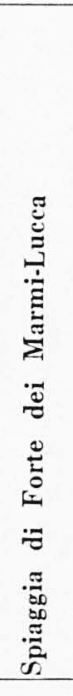 & 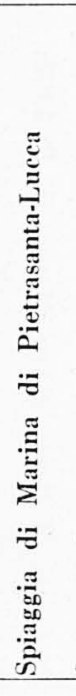 & 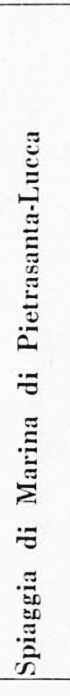 & 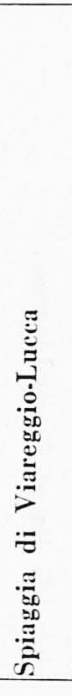 & 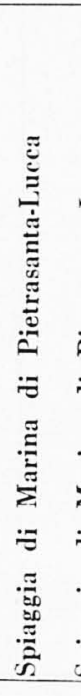 & 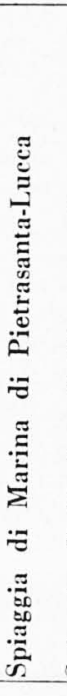 & 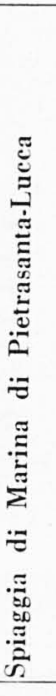 & 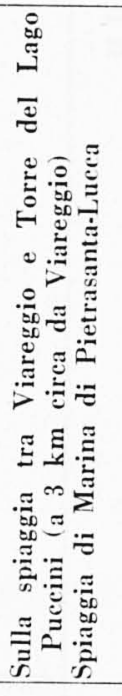 & 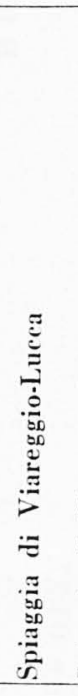 & 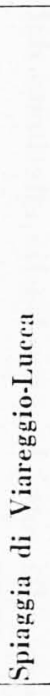 \\
\hline ерәчәs в & : & $\stackrel{\dddot{T}}{\longleftarrow}$ & 荌 & 홍 & & $\stackrel{8}{!}$ & $\widetilde{6}$ & פ্ & 총 & Cִ & 菅 & $\sum_{\overparen{L}}$ & $\stackrel{0}{10}$ & 产 \\
\hline әuтр.ло_p $\mathrm{N}$ & $\dddot{\sim}$ & $ت$ & 12 & $\stackrel{0}{\sim}$ & $\check{-}$ & $\stackrel{\infty}{\sim}$ & 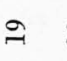 & ๙ิ & $\bar{ง}$ & $\approx$ & ai & $\vec{A}$ 瓷 & $\stackrel{i}{\circ}$ & 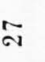 \\
\hline
\end{tabular}




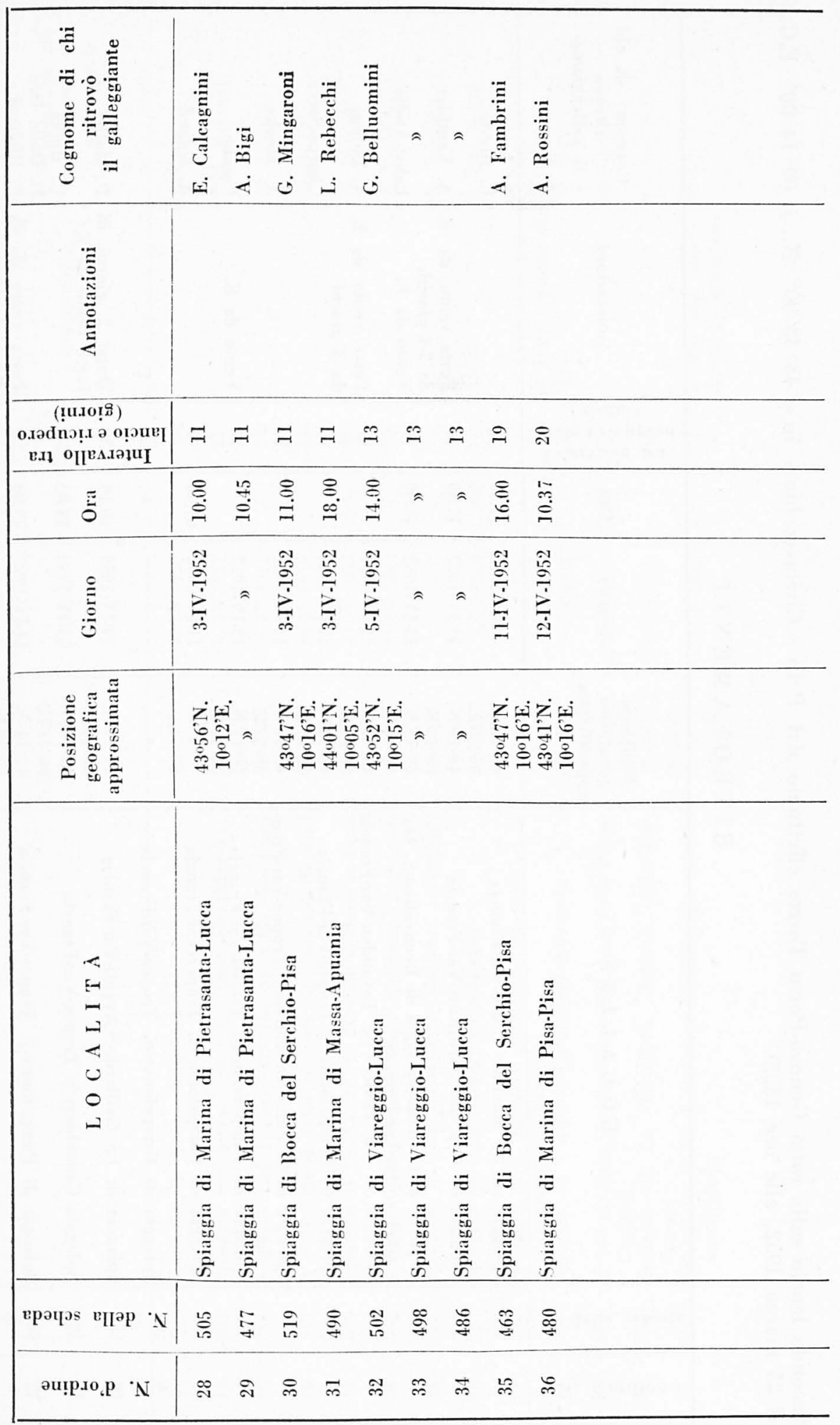


Secondo lancio sulla rotla Genova-Porto Torres effettuato dal P/fo "Civitavecchia " in $\left(\rho 43^{\prime \prime} 43^{\prime} 00^{\prime \prime} N . N^{\prime} \quad 09^{\prime \prime} 14^{\prime} 00^{\prime \prime}\right.$ E.G., il 25 marzo 1952, alle ore 15.20.

\section{R I T R O V A M E T I}

\begin{tabular}{|c|c|c|c|c|c|c|c|c|}
\hline 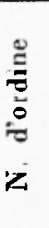 & 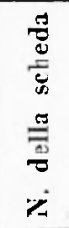 & $\mathrm{L} \mathbf{O} \boldsymbol{C} \boldsymbol{A} \mathrm{L}$ I $\mathrm{T} \dot{\Lambda}$ & $\begin{array}{l}\text { Posizione } \\
\text { geografiea } \\
\text { approssimata }\end{array}$ & Giorno & Orá & 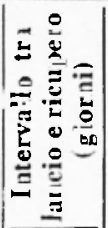 & Annotazioni & $\begin{array}{c}\text { Cognome di chi } \\
\text { ritrovó } \\
\text { il galleggiante }\end{array}$ \\
\hline 1 & 558 & Spiaggia di S. Maxime s/Mer-Var-Francia & $\begin{array}{l}43^{\circ} 18^{\prime} \mathrm{N} . \\
06^{\circ} 39^{\prime} \mathrm{E}\end{array}$ & 12-IV.1952 & 13.00 & 18 & & P. Bretti \\
\hline 2 & 599 & Spiaggia di Cap des Sardinaux-Var-Francia & $\begin{array}{l}43^{\circ} 19^{\prime} \mathrm{N} \text {. } \\
06^{\prime \prime} 40^{\prime} \mathrm{E} .\end{array}$ & 13-IV-1952 & 17.30 & 19 & $\begin{array}{l}\text { Forte vento da } \mathrm{E} . \\
\text { da } 3.4 \text { giorni. }\end{array}$ & A. Vanthier \\
\hline 3 & 5.54 & $\begin{array}{l}\text { A pochi metri dalla spiaggia di Beauvallon-S. Ma- } \\
\text { ximc s/Mer-Var-Francia }\end{array}$ & $\begin{array}{l}43^{\prime \prime} 18^{\prime} \mathrm{N} \\
06^{\prime \prime} 38^{\prime} \mathrm{E}\end{array}$ & 13-I V-1952 & 18.00 & 19 & Vento da E. & Labat-Andia \\
\hline 4 & 548 & Spiaggia di La Favière-Le Lavandou-Var-Francia & 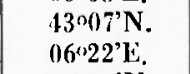 & 13-IV-1952 & & 19 & $\begin{array}{l}\text { Forte vento da } \mathrm{E} \text {. } \\
\text { da } 3 \text { giorni }\end{array}$ & A. Defour \\
\hline 5 & 5.57 & Spiaggia di Pampelonne-S. Tropez-Var-Francia & $\begin{array}{l}43^{\circ} 14^{\prime} \mathrm{N} \text {. } \\
06^{\circ} 40^{\prime} \mathrm{E} \text {. }\end{array}$ & 13-IV-1952 & & 19 & & Charpe-Serre \\
\hline 6 & 597 & $\begin{array}{l}\text { Spiaggia di S. Pons les Mures-S. Tropez-Var-Fran- } \\
\text { rial }\end{array}$ & $\begin{array}{l}43^{\prime \prime} 16^{\prime} \mathrm{N} \\
06^{\prime} 35^{\prime} \mathrm{E}\end{array}$ & 13-IV-1952 & & 19 & & A. Meeks \\
\hline 7 & 573 & Spiaggia di Pampelonne-S. Troper-Var-Francia & $\begin{array}{l}43^{\circ} 14^{\prime} \mathrm{N} \text {. } \\
06^{\circ} 40^{\prime} \mathrm{E} \text {. }\end{array}$ & 13-IV-1952 & & 19 & Vento da E. & Combe \\
\hline 8 & 565 & Spiaggia di Pampelonne-S. Tropez-Var-Francia & ” & $14-1 \mathrm{~V} \cdot 1952$ & 07.00 & 20 & & A. Guaud \\
\hline 9 & 596 & Spiaggia di Pampelonne-S. Tropez-Var-Francia & " & 》 & 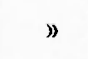 & 20 & & \\
\hline 10 & 556 & Spiaggia lli La Gaillarde-S.Aygulf-Var-Francia & $\begin{array}{l}43^{\prime}, 2+\mathrm{N} . \\
06^{\prime}, 4 t^{\prime} \mathrm{E} .\end{array}$ & $14-1 \mathrm{~V} \cdot 1952$ & 10.10 & 20 & $\begin{array}{l}\text { Dopo } 2 \text { giorni di } \\
\text { forte vento da } E \text {. }\end{array}$ & P. Mollet \\
\hline 11 & 588 & Spiaggia Canmeliers-S. Tropez-Var-Francia & $\begin{array}{l}43^{\prime \prime} 16^{\circ} \mathrm{N} \text {. } \\
06^{\circ}+\mathrm{l}^{\prime} \mathrm{E} .\end{array}$ & 14-IV-1052 & 17.00 & 20 & & $\begin{array}{l}\text { C. Bucci } \\
\text { L. Dalle Ore }\end{array}$ \\
\hline 12 & 561 & Spiaggia di Pampelonne-S. Tropez-Var-Francia & $\begin{array}{l}43^{\circ} 14^{\prime} \mathrm{N} \text {. } \\
06^{\circ} 40^{\prime} \mathrm{E} .\end{array}$ & 14.IV.1952 & 19.00 & 20 & \begin{tabular}{|l} 
Forte vento da E. \\
dal 9 al 14 aprile.
\end{tabular} & P. Richard \\
\hline
\end{tabular}




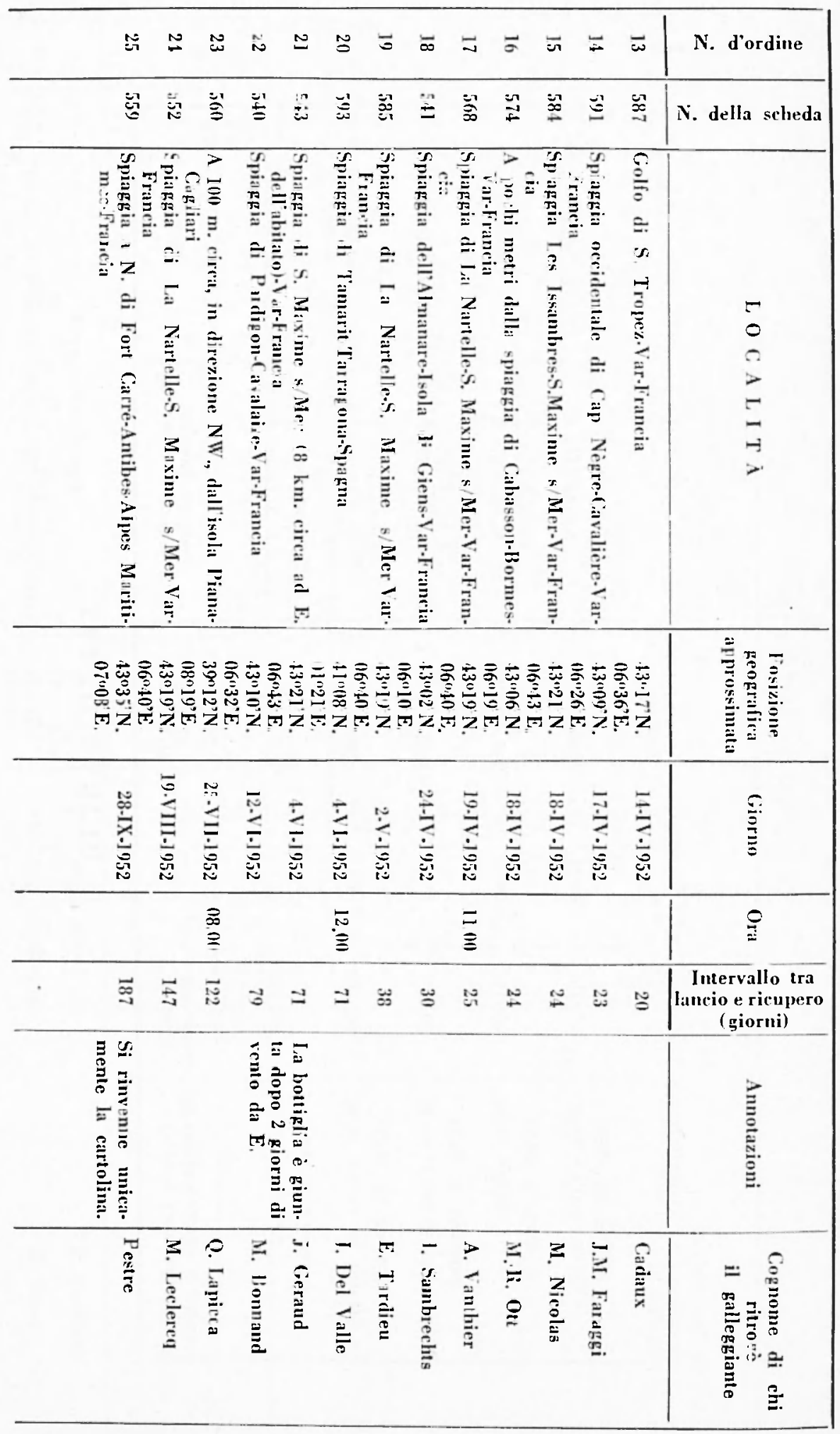


TERZO LA NCIO

\begin{tabular}{|c|c|c|c|c|c|}
\hline Rotta & $\begin{array}{l}\text { Posizione geografica della } \\
\text { stazione di laneio }\end{array}$ & Giorno & Ora & $\begin{array}{c}\text { Numero } \\
\text { delle schede }\end{array}$ & $\begin{array}{l}\text { Nave e Comandante } \\
\text { da cui fu eseguito il lancio }\end{array}$ \\
\hline Cagliari-Trapani & $\begin{array}{ll}\varphi & 38^{\circ} 35^{\prime} 15^{\prime \prime} \mathrm{N} . \\
\lambda & 10^{\prime \prime} 18^{\prime}\left(10^{\prime \prime} \text { E.G. }\right.\end{array}$ & 2 Luglio 1952 & 00.15 & $701-750$ & $\begin{array}{l}\text { P/fo "Torres" } \\
\text { (Cap. R. Rinaudo) }\end{array}$ \\
\hline Napoli-Cagliari & $\begin{array}{ll}\uparrow & 39^{\circ} 53^{\prime} 18^{\prime \prime} \mathrm{N} \\
\lambda & 11^{\circ} .13^{\prime} 18^{\prime \prime} \mathrm{E} . \mathrm{G}\end{array}$ & 15 Luglio 1952 & 20.30 & $601-750$ & $\begin{array}{l}\text { M ve "Citti di Livorno" } \\
\text { (Cap. M. Napoli) }\end{array}$ \\
\hline Civitavecchia-Olbia & $\begin{array}{ll}\text { if } & 11^{\circ} 30^{\prime} 00^{\prime \prime} \mathrm{N} . \\
\lambda & 10^{\circ} \cdot 10^{\prime} 00^{\prime \prime} \mathrm{E} . \mathrm{G} .\end{array}$ & 2 Luglio 1952 & 00.30 & $651-700$ & $\begin{array}{l}\text { M/ve "Citta di Alessandr." } \\
\text { (Cap. A. Damiani) }\end{array}$ \\
\hline Livorno-Bastia & $\begin{array}{ll}\varphi & 13^{\circ} 05^{\prime} 00^{\prime \prime} \mathrm{N} \\
i & 09^{\circ} 58^{\circ}\left(00^{\prime \prime} \mathrm{E} . \mathrm{G} .\right.\end{array}$ & 29 Giugno 1952 & 03.30 & $751-800$ & $\begin{array}{l}\text { M/ve "Olbia " } \\
\text { (Cap. A. Csepely) }\end{array}$ \\
\hline Genova-Porto Torres & $\begin{array}{ll}\varphi & 43^{\circ} 30^{\prime} 00^{\prime \prime} \mathrm{N} \\
\text { t. } & 08^{\circ} \cdot 14^{\prime} 00^{\prime \prime} \mathrm{E} . \mathrm{G}\end{array}$ & 1 Luglio 1952 & 17.00 & $301 \cdot 850$ & $\begin{array}{l}\text { M/ve “Civitavecchia " } \\
\text { (Cap. M. Poggi) }\end{array}$ \\
\hline
\end{tabular}




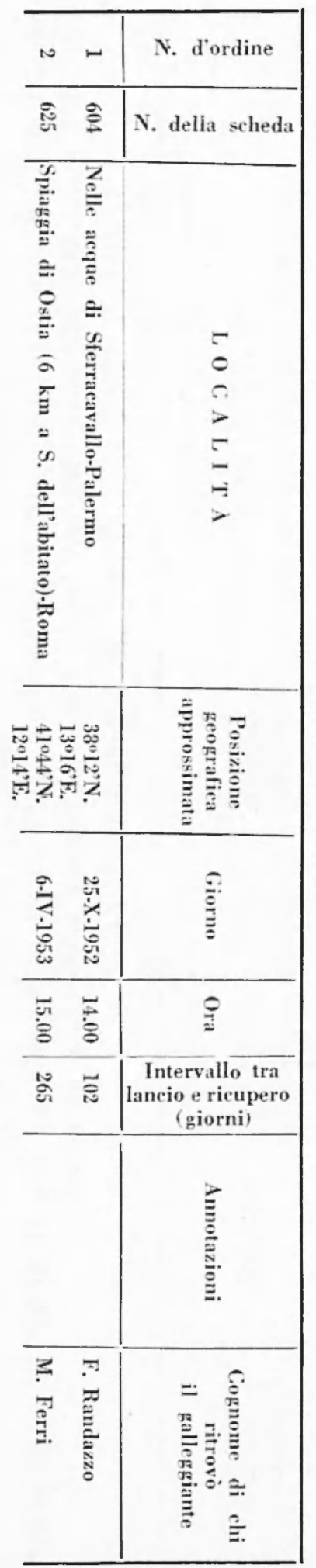

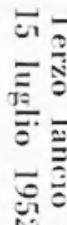
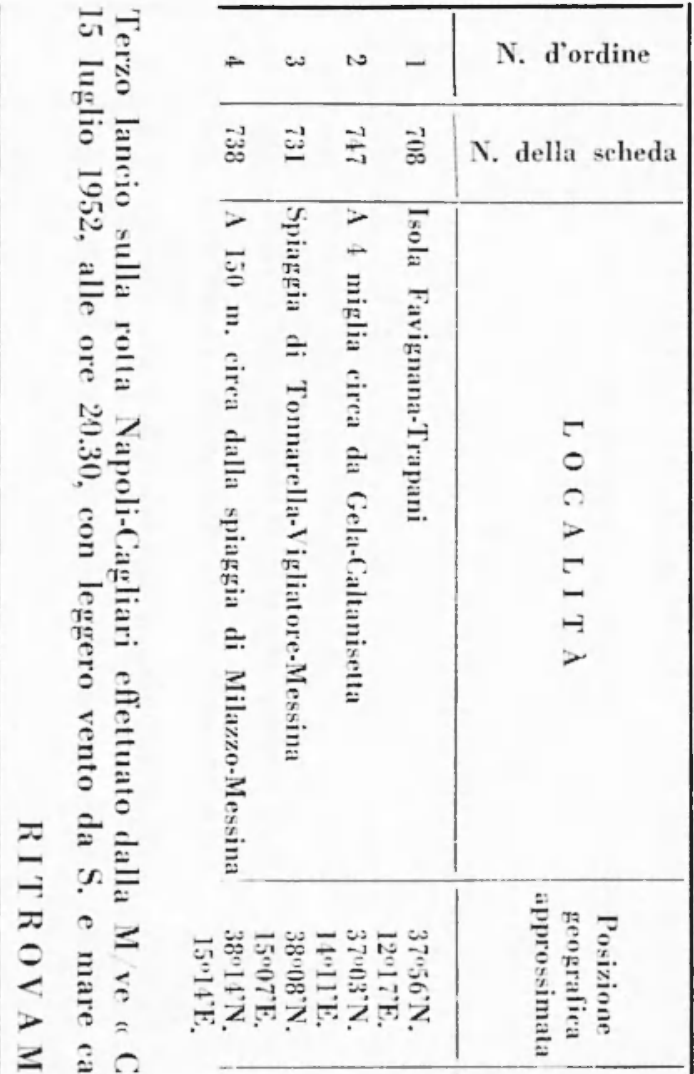

군?

ㄱ.

를

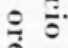

\& ب

$\stackrel{5}{=}$

管

$-\stackrel{\frac{0}{\overrightarrow{0}}}{\stackrel{\vec{*}}{*}}$

$\approx 5$

ثิ

5

$\overrightarrow{0}$

=

学约

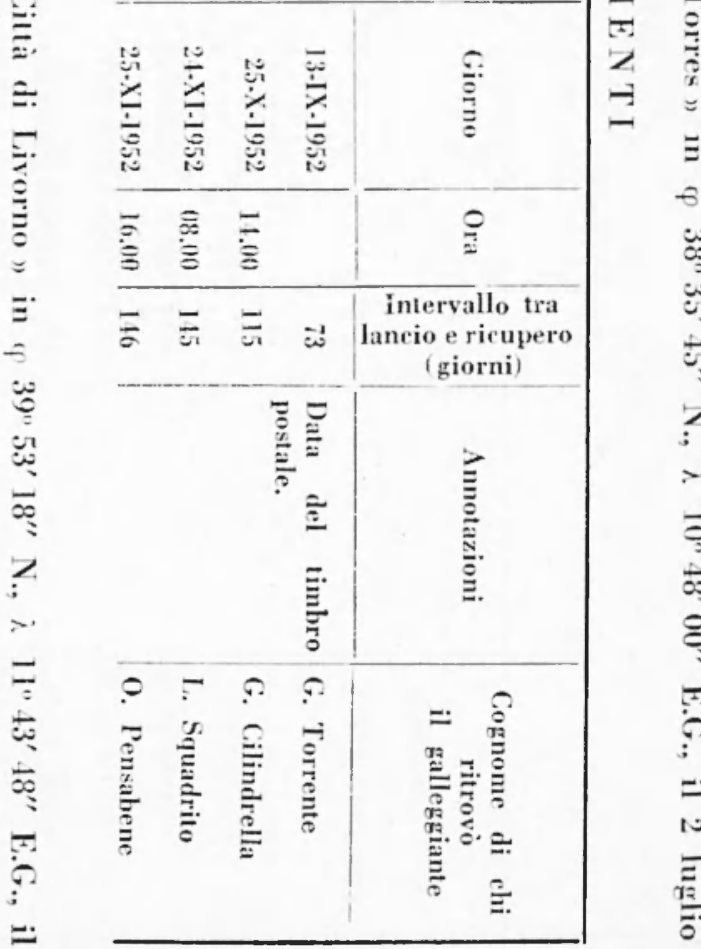


Terzo lancio sulla rotla Civilavecchia-Olhia effettuato dalla M/ve "Citlà di Alessandria " in $\varphi 47^{\circ} 30^{\prime} 00^{\prime \prime}$ N., $\lambda 10^{\circ} 40^{\prime} 00^{\prime \prime}$ E.G., il 2 luglio 1952, alle ore 00.30, con brezza fresca da Ponente e mare appena mosso.

\section{R I T R OV A M E N T I}

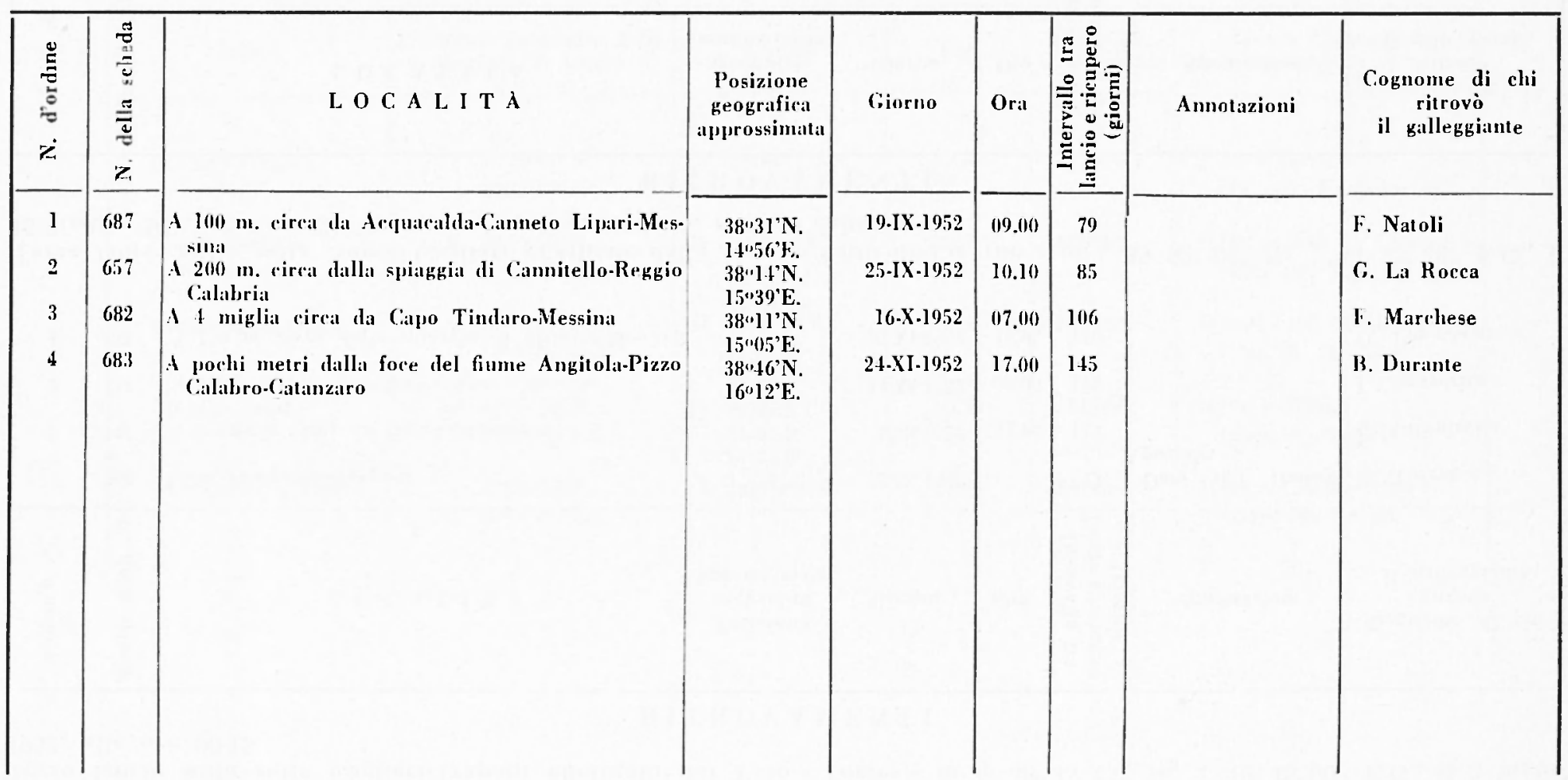


Terzo lancio sulla rotta Livorno-Bastia effettuato dalla $\mathrm{M}$ / ve "Olbia " in $\uparrow 43^{\circ} 05^{\prime} 00^{\prime \prime} \mathrm{N} ., \lambda .09^{\circ} 58^{\prime} 00^{\prime \prime}$ E.G., il 29 giugno 1952 , alle ore 03.30 .

\section{RITROVA M E T I}

\begin{tabular}{|c|c|c|c|c|c|c|c|c|}
\hline 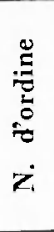 & 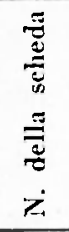 & $\mathrm{L} O \mathrm{C} A \mathrm{~L} I \mathrm{~T} A$ & $\begin{array}{c}\text { Posizione } \\
\text { geografica } \\
\text { approssimata }\end{array}$ & Giorno & Ora & 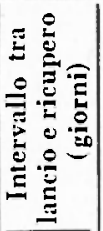 & Annotazioni & $\begin{array}{c}\text { Cognome di chi } \\
\text { ritrovò } \\
\text { il galleggiante }\end{array}$ \\
\hline 1 & 790 & Spiaggia di Prigioni-Bastia-Corsica & $\begin{array}{l}42 \circ 43 \mathrm{~N} \\
09027 \mathrm{E}\end{array}$ & 14-VII-1952 & 14.30 & 15 & & L. Cavallaro \\
\hline 2 & 757 & A $20 \mathrm{~m}$. circa dal lido di S. Iacopo-Livorno & $\begin{array}{l}43^{\circ} 3 \mathrm{l}^{\prime} \mathrm{N} . \\
10018^{\prime} \mathrm{E} .\end{array}$ & 7-VIII.1952 & 11.00 & 39 & & M. Gianetti \\
\hline 3 & 751 & Spiaggia di Pianosa Isola-Livorno & $\begin{array}{l}42 \circ 36 \mathrm{~N} \text {. } \\
10 \cdot(0.4 \mathrm{E} .\end{array}$ & 8-VIIl-1952 & 08.30 & 40 & & G. Levanto \\
\hline 4 & 77.1 & Spiaggia di Procchio-Isola d'Elba-Livorno & $\begin{array}{l}42047 \cdot \mathrm{N} \\
10^{\circ} 15 \cdot \mathrm{E}\end{array}$ & 8-VIII-1952 & 18.00 & 40 & & A. Paolini \\
\hline 5 & 761 & Canale di l’iombino-Livorno & $\begin{array}{l}42055 ' \mathrm{~N} \text {. } \\
10 \cdot 31 ' \mathrm{E} \text {. }\end{array}$ & 10- I III-1952 & 19.00 & 42 & Vento di Muestrale. & R. Bacei-G. Bianchi \\
\hline 6 & 799 & $\begin{array}{l}\text { A } 500 \text { m. eirea dalla spiaggia di Finale Ligure-Sat } \\
\text { vonal }\end{array}$ & $\begin{array}{l}44^{\circ} 10^{\prime} \mathrm{N} \text {. } \\
08^{\circ} 2 \mathrm{l}^{\prime} \mathrm{E} \text {. }\end{array}$ & 12-VIII-1952 & 16.30 & 44 & & M. Costamagna \\
\hline 7 & 778 & Sulla spiaggia tra Tirrenia e Calambrone-Pisa & $\begin{array}{l}43^{\circ} 37^{\prime} \mathrm{N} \\
10^{\circ} 17^{\prime} \mathrm{E}\end{array}$ & 16-VIII-1952 & 07.00 & 48 & & S. Nocohi \\
\hline 8 & 788 & Fore del fume Alesani-S. Giuliano-Corsica & $\begin{array}{l}42^{\circ} 18^{\prime} \mathrm{N} \\
0903+4^{\prime} \mathrm{E}\end{array}$ & 25.VIII.1952 & 16.00 & 57 & & J. Ottomani \\
\hline 9 & 783 & Spiaggia di Tor Materno-Anzio-Roma & $\begin{array}{l}41 \cdots, 4 l^{\prime} \mathrm{N} \text {. } \\
12^{\circ} 233^{\prime} \mathrm{E} \text {. }\end{array}$ & 20-IX-1952 & 07.30 & 83 & $\begin{array}{l}\text { Forte vento da } \mathrm{W} . \\
\text { da } 2 \text { giorni. }\end{array}$ & V. Valentino \\
\hline 10 & 798 & I.ido d'Ardea-Roma & $\begin{array}{l}41035 \mathrm{~N} . \\
12^{\circ} 30^{\prime} \mathrm{J} .\end{array}$ & $8-X-1952$ & 09.00 & 101 & $\begin{array}{l}\text { Forti venti di Mae. } \\
\text { strale. }\end{array}$ & F. De Clemente \\
\hline 11 & 752 & $\begin{array}{l}\text { A } 10 \text { miglia circa da Capo Sottile, in direzione } \\
\text { dell'Isola dei Galli-Praiano-Salerno }\end{array}$ & $\begin{array}{l}40^{\circ} 32 ' \mathrm{~N} \\
14^{\circ} 20^{\prime} \mathrm{E}\end{array}$ & $31 \cdot X \cdot 1952$ & 11.00 & 124 & & L. Irace \\
\hline 12 & 796 & Spiaggia di Tarquinia-Porto Gravina-Viterbo & $\begin{array}{l}+12^{\circ} 1+\mathrm{N} . \\
11^{\circ}+2^{\prime} \mathrm{E} .\end{array}$ & 24-XI-1952 & 10.00 & 148 & & G. Tumarelli \\
\hline 13 & 784 & Gollo di Gava-Aiaccio-Corsica & $\begin{array}{l}4{ }^{\circ} \cdot 54^{\prime} \mathrm{N} \text {. } \\
08^{\circ} 42^{\prime} \mathrm{E} \text {. }\end{array}$ & 8-III-1953 & & 252 & & F. Pischi \\
\hline
\end{tabular}


Terzo lancio sulla rolla Genova-Porto Torres effettuato dalla M/ve "Civitavecchia " in $\varphi 43^{\prime \prime} 30^{\prime} 00^{\prime \prime} \mathrm{N} ., \lambda .08^{\prime \prime} 44^{\prime} 00^{\prime \prime}$ E.G., il $1^{\circ}$ luglio 1952, alle ore 17.00 .

\section{R I T R O A M E N T I}

\begin{tabular}{|c|c|c|c|c|c|c|c|c|}
\hline 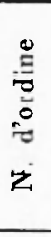 & 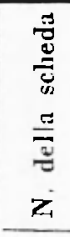 & 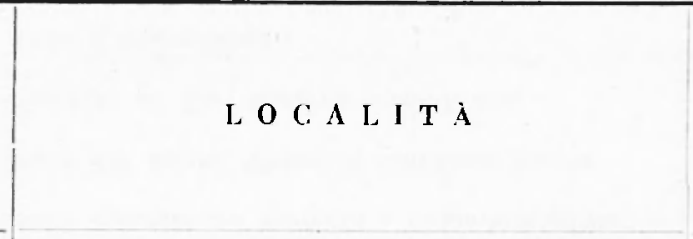 & $\begin{array}{l}\text { Posizione } \\
\text { geografical } \\
\text { approssimalta }\end{array}$ & Giorno & Ora & 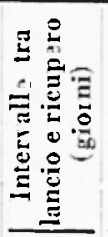 & Annotazioni & $\begin{array}{c}\text { Cognome di chi } \\
\text { ritrovi) } \\
\text { il galleggiante }\end{array}$ \\
\hline 1 & 80.4 & $\begin{array}{l}\text { Tra l'Isola S. Marguerite e l'Isola S. Honorat-Can- } \\
\text { nes-Alpes Maritimes-Francia }\end{array}$ & $\begin{array}{l}43^{\prime \prime} 31^{\prime} \mathrm{N} \\
07 \cdot\left(03^{\prime} \mathrm{E}\right.\end{array}$ & 15.111 .1952 & 16.00 & 14 & & Herbein \\
\hline 2 & 849 & $\begin{array}{l}\text { Spiaggia meridionale dell'Isola } S \text {. Marguerite-Can- } \\
\text { nes-Alpes Maritimes-Francia }\end{array}$ & $\begin{array}{l}43^{\circ} 31^{\prime} \mathrm{N} \\
07^{\circ}(0)^{\prime} \mathrm{E}\end{array}$ & 16.VII.1952 & & 15 & & Iolnamnen \\
\hline 3 & 803 & Spiaggia di Gigaro-La Croix Valmer-Var-Francia & $\begin{array}{l}13011 ' \mathrm{~N} . \\
066^{\prime \prime} 33^{\prime} \mathrm{E} .\end{array}$ & $17 . \mathrm{VII.1952}$ & & 16 & & C. S. Plancat \\
\hline 4 & 805 & $\begin{array}{l}\text { Tra l'Isola S. Marguerite e l'Isola S. Honorat-Can- } \\
\text { nes- } A \text { lpes Maritimes-Francial }\end{array}$ & $\begin{array}{l}13^{\prime \prime} 31^{\prime} \mathrm{N} \text {. } \\
07 \cdot 03^{\circ} \mathrm{E} \text {. }\end{array}$ & $17-\mathrm{VII}-1952$ & & 16 & & G. Russel \\
\hline 5 & 838 & $\begin{array}{l}\text { Spiaggia di Capo Les Salins-S. Troper-Var-Fran- } \\
\text { cia }\end{array}$ & $\begin{array}{l}+3^{\prime \prime}\left(16^{\prime} \mathrm{N}\right. \\
06^{\prime \prime} 12 \cdot \mathrm{E} \text {. }\end{array}$ & $18-\mathrm{VII} \cdot 1952$ & 12.30 & 17 & & P. Savini \\
\hline 6 & $8+1$ & Spiaggia di La Croix Valmer-Var-Francia & $\begin{array}{l}43^{\circ} 11^{\prime} \mathrm{N} \text {. } \\
06^{\prime \prime} 33^{\prime} \mathrm{E} .\end{array}$ & $18 \cdot \mathrm{VII} \cdot 1052$ & & 17 & & $\boldsymbol{W}$. Riedes \\
\hline 7 & 806 & $\begin{array}{l}\text { A } 50 \text { m. rirca dalla spiagria di Pampelonne.S. Tro- } \\
\text { pez-Var-l rancial }\end{array}$ & $\begin{array}{l}+3^{\circ} 11+\mathrm{N} . \\
106^{\circ} 10^{\prime} \mathrm{E} .\end{array}$ & $18 \cdot V 1 I-1952$ & & 17 & & M. Calsanel \\
\hline 8 & 809 & $\begin{array}{l}\text { A } 200 \text { m. rirca dal faro di Camarat-S. Tropez-lar- } \\
\text { Francia }\end{array}$ & $\begin{array}{l}+3^{\circ} 12 \mathrm{~N} \\
06^{\prime \prime}+1 \mathrm{E}\end{array}$ & $19 . V 11.1952$ & & 18 & Forte vento da E. & P. Girardet \\
\hline 9 & 801 & $\begin{array}{l}12 \mathrm{~km} \text {. Circa dalla spiaggia de La Nartelle-S. Ma- } \\
\text { xime s, Mer-Var-Francia }\end{array}$ & $\begin{array}{l}\left.43^{\prime \prime}\right] 9^{\circ} \mathrm{N} \\
06^{\circ}+12^{\circ} \mathrm{E}\end{array}$ & 20. VII.1952 & & 19 & Vento da E. & Bonmartin \\
\hline 10 & 828 & Spiaggia di Capo Camalat-S. Tropez-Vall-Framcia & $\begin{array}{l}43^{\circ} 12^{\circ} \mathrm{N} . \\
06^{\circ}+11^{\circ} \mathrm{E} .\end{array}$ & $20 . \mathrm{VII}-1952$ & 16.00 & 19 & & G. Leocard \\
\hline 11 & 821 & Nel porto dei Mourillons-Joulon-Var-Francia & $\begin{array}{l}43^{\circ} 06^{\circ} \mathrm{N} \\
050.57 \mathrm{~F}\end{array}$ & $22 . \mathrm{VII} .1952$ & 13.30 & 21 & Vento da E. & P. Parluet \\
\hline 12 & 821 & Spiaggia di Prola-Agay.Var-Francia & $\begin{array}{l}43^{\circ} 26^{\circ} \mathrm{N} \\
066^{\circ}, 52^{\circ} \mathrm{K}\end{array}$ & 22.111 .1952 & 16.30 & 21 & Vento da E. & Dufourd \\
\hline
\end{tabular}




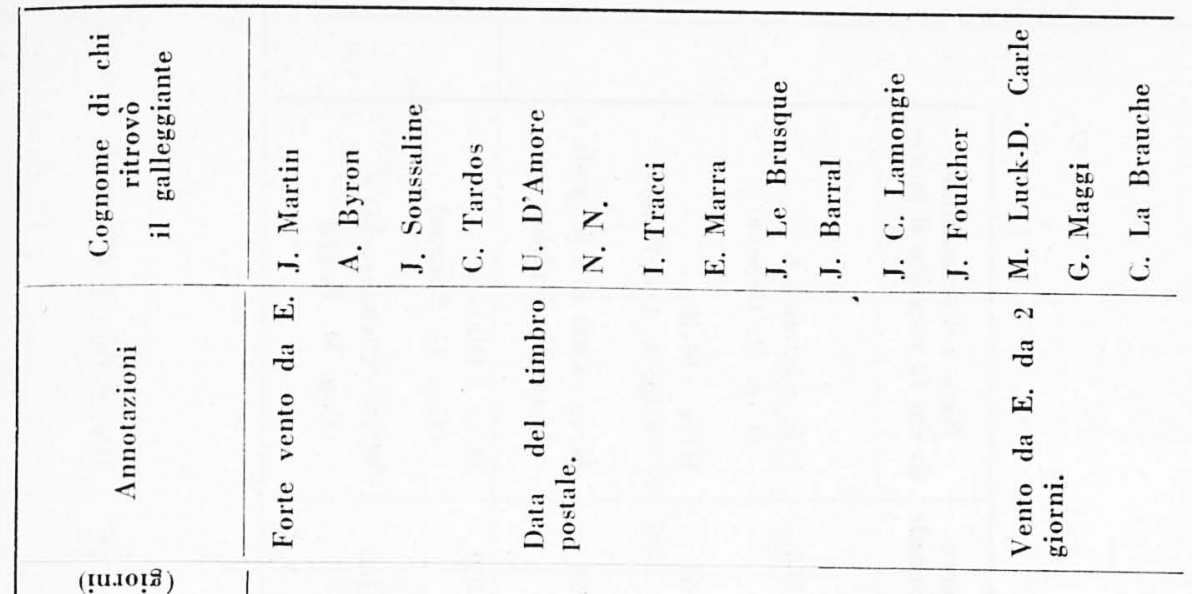

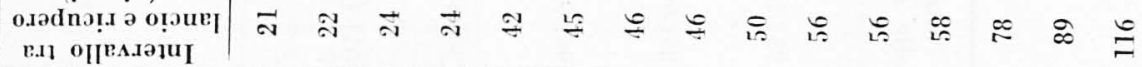

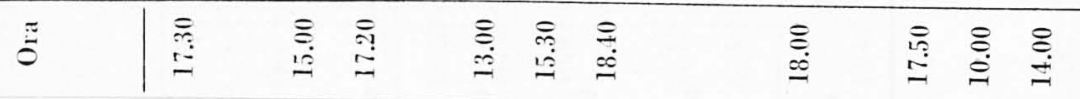

$\stackrel{\varrho}{\vdots}$

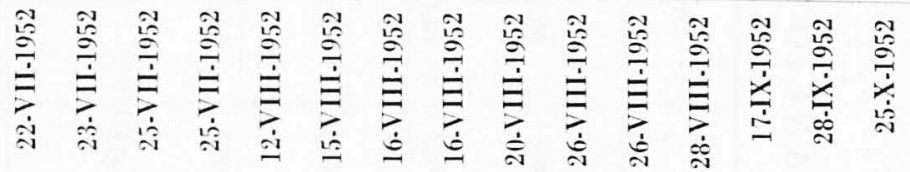

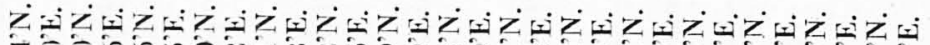

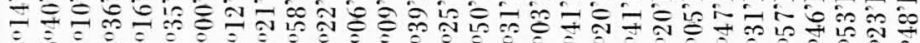

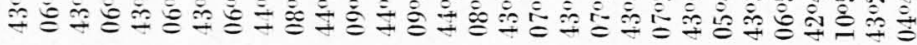

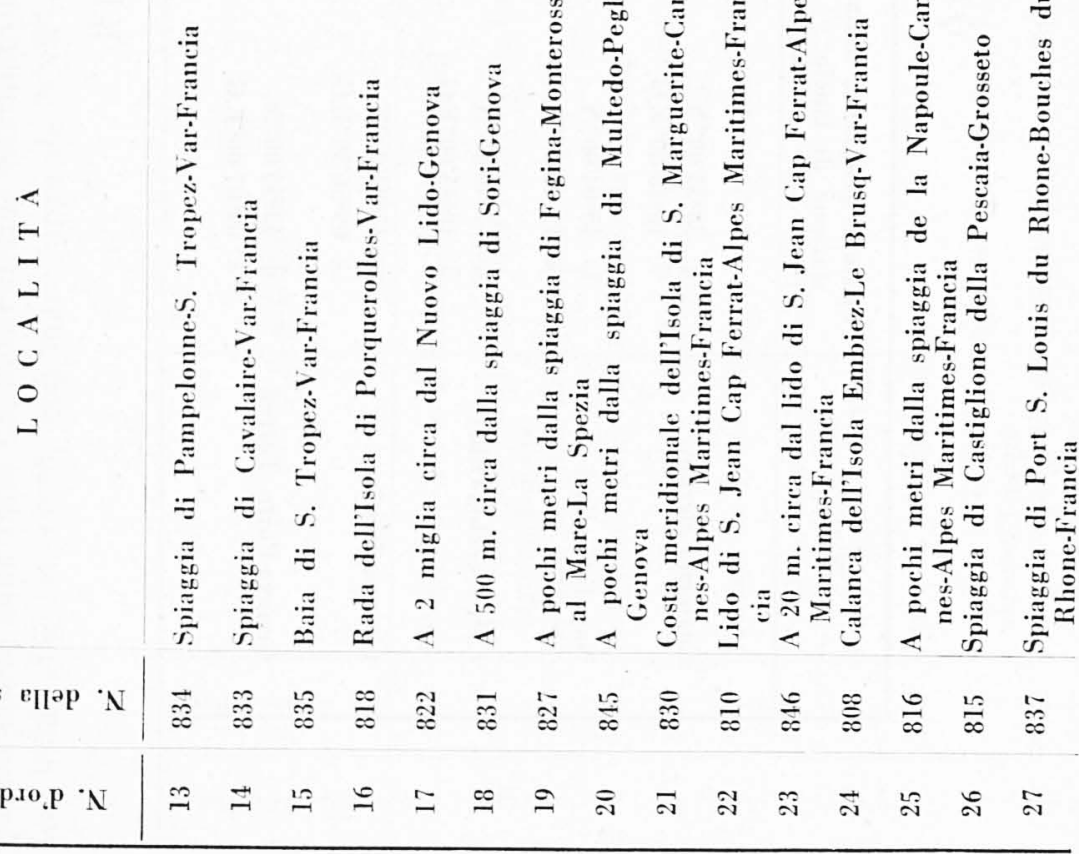


Q UARTO LANCIO

\begin{tabular}{|c|c|c|c|c|c|}
\hline Rotta & $\begin{array}{l}\text { Posizione geografica della } \\
\text { stazione di lancio }\end{array}$ & Giorno & Ora & $\begin{array}{c}\text { Numero } \\
\text { delle schede }\end{array}$ & $\begin{array}{l}\text { Nave e Comandante } \\
\text { da cui fu eseguito il lancio }\end{array}$ \\
\hline Cangliari-Palermo & $\begin{array}{l}\text { y. } 38^{\prime \prime} 35^{\prime} 00^{\prime \prime} \mathrm{N} . \\
\text { i. } 10^{\prime \prime} .18^{\prime} 00^{\prime \prime} \mathrm{E} . \mathrm{G} .\end{array}$ & 6 Novembre 1952 & 00.30 & 951.1000 & $\begin{array}{l}\text { P/fo “Jchnusa » } \\
\text { (Cap. R. Genova) }\end{array}$ \\
\hline Napoli-Cagliari & 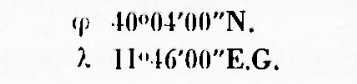 & 4 Novembre 1952 & 2.1 .00 & $851-900$ & $\begin{array}{l}\text { M/ve "Sicilia " } \\
\text { (Cap. A. Del Pino) }\end{array}$ \\
\hline Civitavecchia-Olbia & $\begin{array}{l}\text { if }+1^{\circ} 30^{\prime} 00^{\prime \prime} \mathrm{N} . \\
\text { h. } 10^{\circ}+11^{\prime} 00^{\prime \prime} \text { E.G. }\end{array}$ & 5 Novembre 1952 & 00.30 & $901 \cdot 950$ & $\begin{array}{l}\text { M/ve "Città di Alessandr." } \\
\text { (Cap. V. Sepirh) }\end{array}$ \\
\hline Livorno-Bastia & $\begin{array}{l}\psi 43^{\prime} 0.4^{\prime} 00^{\prime \prime} \mathrm{N} . \\
\lambda .09 \cdot 56^{\prime} 30^{\prime \prime} \mathrm{E} . \mathrm{G} .\end{array}$ & 2 Novembre 1952 & 03.20 & 1001.1050 & $\begin{array}{l}\text { M/ve “Olbia " } \\
\text { (Cap. G. Somma) }\end{array}$ \\
\hline Genova-Porto Torres & $\begin{array}{ll}\text { if } & 43^{\circ} .43^{\prime} 00^{\prime \prime} \mathrm{N} . \\
\text { i. } & 09^{\circ} 07^{\prime} 00^{\prime \prime} \mathrm{E} . \mathrm{G} .\end{array}$ & + Novembre 1952 & & $1051 \cdot 1100$ & $\begin{array}{l}\text { Mive "Civitavecchia D } \\
\text { (Cap. M. Poggi) }\end{array}$ \\
\hline
\end{tabular}


Quarto lancio sulla rolla Cagliari-Palermo effeltuato dal P, fo "Iclinusa " in " $p 38^{\circ} 35^{\prime} 00^{\prime \prime}$ N., $\% 10^{\circ} 48^{\prime} 00^{\prime \prime}$ F.G.: il 6 novembre 1952, alle ore 00.30, con mare lungo da NNW e vento da NE forza 2.

\section{RITROVA MENTI}

\begin{tabular}{|c|c|c|}
\hline 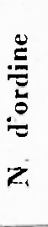 & 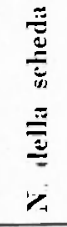 & $\mathrm{L} O \boldsymbol{C} \mathrm{A}$ L I T $\dot{\Lambda}$ \\
\hline 1 & 979 & Spiaggia di Torre Scibiliana-Marsala-Trapani \\
\hline 2 & 995 & $\begin{array}{l}\text { Ad } 1 \mathrm{~km} \text {. circa dal faro di Punta Marsala-Isola Fa- } \\
\text { vignana-Tra!̣ani }\end{array}$ \\
\hline 3 & 98.5 & Spiaggia di Torre Scibiliana-Marsala-Trapani \\
\hline 4 & 983 & Spiaggia di Torre Scibiliana-Marsala-Trapani \\
\hline 5 & 998 & Spiaggia di Torre Scibiliana-Marsala-Trapani \\
\hline 6 & 962 & Spiazgia di Torre Scibiliana-Marsala-Trapani \\
\hline 7 & 951 & $\begin{array}{l}\text { A } 500 \mathrm{~m} \text {. circa dalla spiaggia di Torre Sribiliana- } \\
\text { Marsala-Trapani }\end{array}$ \\
\hline 8 & 963 & Portu di Marsala-Trapani \\
\hline 9 & 996 & Porto di Marsala-Trapani \\
\hline 10 & 955 & $\begin{array}{l}\text { A } 50 \text { m. cirea dalla spiaggia del Burrone-Isola Fa- } \\
\text { vignana-Trapani }\end{array}$ \\
\hline 11 & 97.4 & $\begin{array}{l}\text { A } 50 \mathrm{~m} \text {. circa dalla spiaggia del Burrone-Isola Fa- } \\
\text { vignana-Trapani }\end{array}$ \\
\hline 12 & 95.4 & $\begin{array}{l}\text { A } 50 \text { m. rirca dalla spiaggia del Burrone-Isola Fa- } \\
\text { vignana-Trapani }\end{array}$ \\
\hline
\end{tabular}

\begin{tabular}{|c|c|c|c|c|c|}
\hline $\begin{array}{c}\text { Posizione } \\
\text { geografical } \\
\text { approssimatal }\end{array}$ & Giorno & Ora & 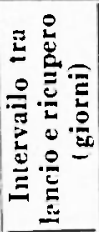 & Annotazioni & $\begin{array}{c}\text { Cognome di chi } \\
\text { ritrovò } \\
\text { il galleggiante }\end{array}$ \\
\hline $37^{\circ} .43$ 'N. & 13-XI-1952 & 06.00 & 7 & & A. Giacalone \\
\hline $\begin{array}{l}12^{\circ} 28^{\prime} \mathrm{E} . \\
3705 .+\mathrm{N}\end{array}$ & 13.XI-1952 & 08.00 & 7 & & G. Patti \\
\hline 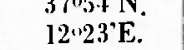 & & & & & 2. \\
\hline $\begin{array}{l}37^{\circ}+3^{\circ} \mathrm{N} . \\
12^{\circ} 28^{\prime} \mathrm{E} .\end{array}$ & 13-XI-1952 & 10.00 & 7 & Sul bagnasciuga & V. Pnlizxi \\
\hline ) & ) & $"$ & 7 & & S. Pisitano \\
\hline$)$ & 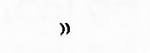 & 10.15 & 7 & Sul bagnasciuga & 1). Pizzolato \\
\hline 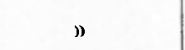 & 1.1.XI-1952 & 09.00 & 8 & & G. Silanos \\
\hline$"$ & ) & 10.00 & 8 & & G. Balestrieri \\
\hline $37 \circ 47 \mathrm{~N}$. & $14-\mathrm{XI} \cdot 1952$ & 15.45 & 8 & & A. Mezzal \\
\hline $\begin{array}{c}12026 \mathrm{E} \\
\Rightarrow\end{array}$ & 15-XI-1952 & 11.00 & 9 & & A. Accardi \\
\hline $\begin{array}{l}37^{\circ} 54^{\circ} \mathrm{N} . \\
12 \circ 22^{\prime} \mathrm{E} .\end{array}$ & $18-X I-19.52$ & 06.00 & 12 & & M. Ritunno \\
\hline$)$ & 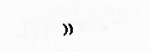 & 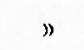 & 12 & & ) \\
\hline$)$ & ) & ) & 12 & & G. Ritunno \\
\hline
\end{tabular}




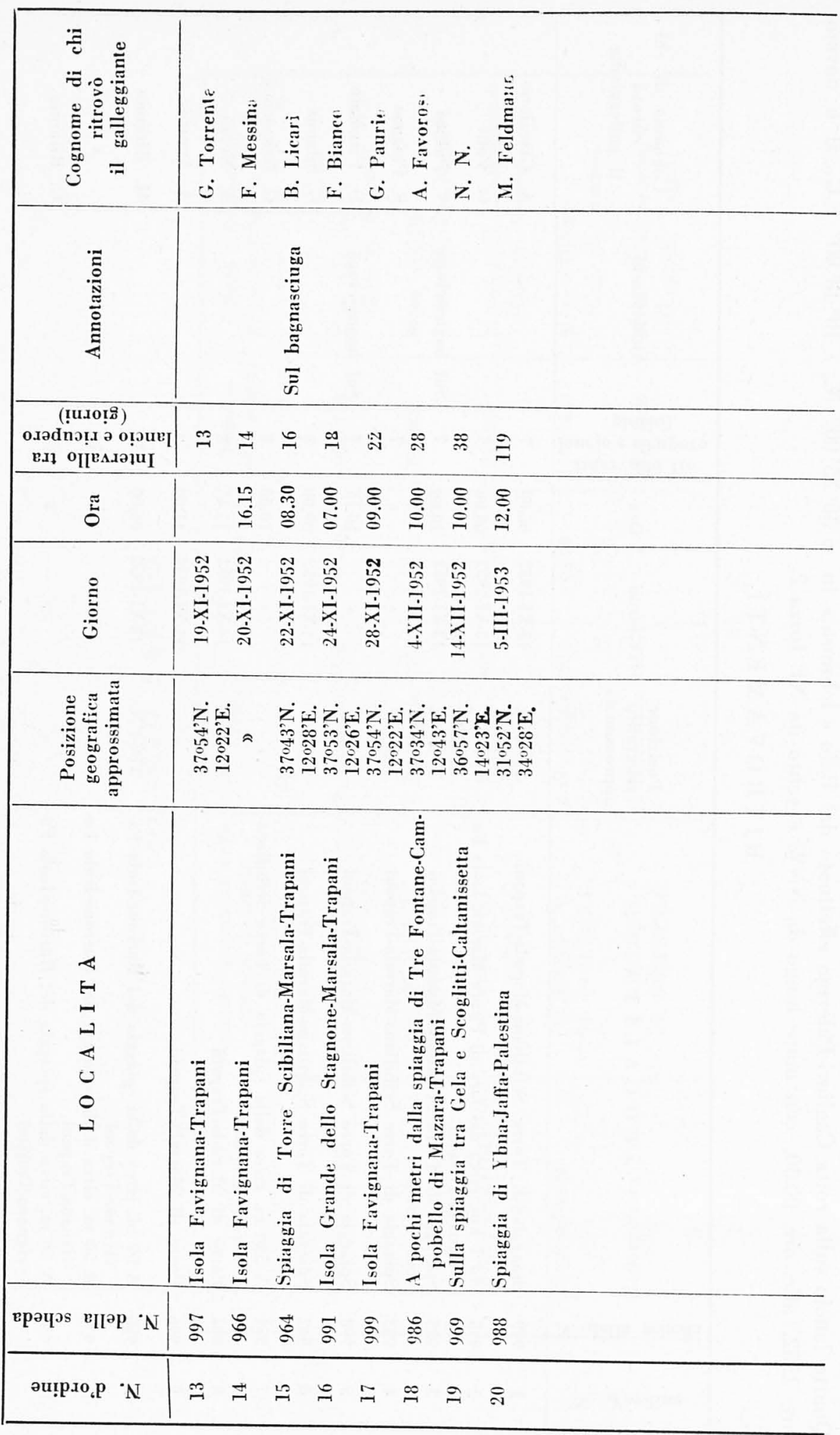


Quarto lancio sulla rotta Napoli-Cagliari eflettuato dalla M/ve "Sicilia " in $\varphi .40^{\circ} 04^{\prime} 00^{\prime \prime}$ N., $7.11^{\circ} 46^{\prime} 00^{\prime \prime}$ E.G., ii 4 novemlore 1952, alle ore 24.00 .

\section{R I T ROVA M N T I}

\begin{tabular}{|c|c|c|c|c|c|c|c|c|}
\hline 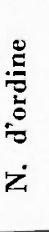 & 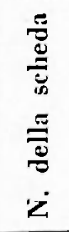 & $\mathrm{L} O C A \mathrm{~L}$ I $\mathrm{T} \dot{\boldsymbol{A}}$ & $\begin{array}{c}\text { Posizione } \\
\text { geografica } \\
\text { approssimata }\end{array}$ & Giorno & Ora & 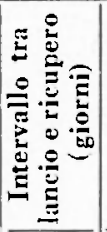 & Annotazioni & $\begin{array}{c}\text { Cognome di chi } \\
\text { ritrovó } \\
\text { il galleggiante }\end{array}$ \\
\hline 1 & 890 & $\begin{array}{l}\text { A } 500 \text { m. circa da Punta Capo Faro-Isola Salina- } \\
\text { Messina }\end{array}$ & $\begin{array}{l}38^{\circ} 35^{\prime} \mathrm{N} \\
14^{\circ} 53^{\prime} \mathrm{E}\end{array}$ & 1.XII-1952 & 14.00 & 27 & \multirow{13}{*}{$\begin{array}{l}\text { Bottiglia rinvenuta } \\
\text { gii rottit. }\end{array}$} & I. Patané \\
\hline 2 & 86.1 & Spiaggia di Aversana-Battipaglia-Salerno & $\begin{array}{l}40032 ' \mathrm{~N} . \\
14055 \mathrm{E}\end{array}$ & 16.XII-1952 & 10.00 & 42 & & G. Castagna \\
\hline 3 & 800 & Spiaggia di Palinuro-Salerno & $\begin{array}{l}40 \cdots 02^{\prime} \mathrm{N} \text {. } \\
15^{\circ} 16^{\prime} \mathrm{E} \text {. }\end{array}$ & 16-XII-1952 & 12.30 & 42 & & A. Erranti \\
\hline 4 & 870 & Spiaggia di Campolongo-Battipaglia-Salerno & $\begin{array}{l}40^{\circ} 39^{\prime} \mathrm{N} \\
14^{\circ} 49^{\circ} \mathrm{E}\end{array}$ & $18 \cdot X 11-1952$ & 08.00 & 44 & & D. Capacchione \\
\hline 5 & 859 & Scogliera di Castrocucco-Maratea-Potenza & $\begin{array}{l}39 \circ 56^{\prime} \mathrm{N} \\
15 \circ 45 \cdot \mathrm{E}\end{array}$ & 18-XII-1952 & 09.00 & 41 & & T. Labanchi \\
\hline 6 & 855 & Spiaggia di Amantea-Cosenza & $\begin{array}{l}39008^{\prime} \mathrm{N} \text {. } \\
16^{\circ} 04^{\prime} \mathrm{E} .\end{array}$ & 18-XII-1952 & 11.00 & 4 & & A. Molinano \\
\hline 7 & 873 & Spiaggia di Agropoli-Salerno & $\begin{array}{l}40021^{\prime} \mathrm{N} \\
15^{\circ} 00^{\prime} \mathrm{E}\end{array}$ & 19.XII-1952 & 07.30 & 45 & & R. Benevento \\
\hline 8 & 875 & Spiaggia di Curinga-Catanzaro & $\begin{array}{l}38^{\circ} \cdot 5 \mathrm{l}^{\prime} \mathrm{N} \text {. } \\
16^{\circ} 13^{\prime} \mathrm{E} \text {. }\end{array}$ & 19-XII-1952 & 08.30 & 45 & & I. Gallo \\
\hline 9 & 892 & Spiaggia di Marina dei Pioppi-Salerno & $\begin{array}{l}40^{\circ} 10^{\prime} \mathrm{N} \text {. } \\
15^{\circ} 05^{\prime} \mathrm{E} \text {. }\end{array}$ & 27-XII-1952 & 13.20 & 53 & & A. D'Alessindro \\
\hline 10 & 874 & Spiaggia di S. Anastasia-Anzio-Roma & $\begin{array}{l}41 \circ 03 ' \mathrm{~N} . \\
12034 ' \mathrm{E} .\end{array}$ & 1.I-1953 & & 58 & & G. Magnanimi \\
\hline 11 & 893 & Spiaggia di Capo Portiere-Latina & $\begin{array}{l}41025^{\prime} \mathrm{N} \\
12^{\circ} 48^{\prime} \mathrm{E}\end{array}$ & $2 . I .1953$ & 09.00 & 59 & & M. Perin \\
\hline 12 & 876 & $\begin{array}{l}\text { A } 2 \text { miglia circa, in direzione } W_{.} \text {dall'Isola Pal- } \\
\text { marola-Latina }\end{array}$ & $\begin{array}{l}40056^{\prime} \mathrm{N} \text {. } \\
122_{4}{ }^{\circ} \mathrm{E} \text {. }\end{array}$ & 15-I-1953 & 10.00 & 71 & & A. Vitiello \\
\hline 13 & 377 & $\begin{array}{l}\text { A pochi metri dalla spiaggia di S. Lncia di Sini- } \\
\text { scola-Nuoro }\end{array}$ & $\begin{array}{l}40^{\circ} 35 ' \mathrm{~N} . \\
09047 \mathrm{E} .\end{array}$ & 10-III-1953 & 11.00 & 126 & & I. Romano \\
\hline
\end{tabular}


Quarto lancio sulla rotta Civitavecehia-Olbia effettuato dalla $\mathrm{M} / \mathrm{ve}$ "Città di Alessandria " in $p 41^{\circ} 30^{\prime} 00^{\prime \prime} \mathrm{N}$., $\lambda 1^{\prime \prime} 4 \mathrm{I}^{\prime} 00^{\prime \prime}$ E.G., il 5 novembre 1952 , alle ore 00.30 , con vento fresco e mare molto agitato da $\mathrm{N}$.

\section{R I T R O V A M E N T I}

\begin{tabular}{|c|c|c|c|c|c|c|c|c|}
\hline 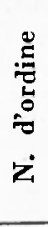 & 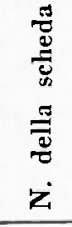 & L O C A L I T $\lambda$ & $\begin{array}{c}\text { Posizione } \\
\text { geografica } \\
\text { approssimata }\end{array}$ & Giorno & Ora & 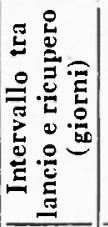 & Annotazioni & $\begin{array}{c}\text { Cognome di chi } \\
\text { ritrovò } \\
\text { il galleggiante }\end{array}$ \\
\hline 1 & 902 & Spiaggia di Feniglia-Orbetello-Grosseto & $\begin{array}{l}42 \circ 25 ' \mathrm{~N} \\
11^{\circ 14} \mathrm{E} .\end{array}$ & $1 \cdot X I I \cdot 1952$ & 06.00 & 26 & & L. Corazzini \\
\hline 2 & 903 & Spiaggia di Feniglia-Orbetello-Grosseto & $»$ & $"$ & $"$ & 26 & & $»$ \\
\hline 3 & 935 & Spiaggia di Feniglia-Orbetello-Grosseto & $»$ & $"$ & 17.00 & 26 & Venti di Sud & P. Reginelli \\
\hline 4 & 918 & Cala dei Santi-Porto Errole-Grosseto & $\begin{array}{l}42^{\prime} 24^{\prime} \mathrm{N} \\
11^{\circ} 13^{\prime} \mathrm{E} \text {. }\end{array}$ & $3-X I 1-1952$ & & 28 & $\begin{array}{l}\text { Scheda ritrovata su- } \\
\text { gli scogli. }\end{array}$ & D. Massandrini \\
\hline 5 & 924 & $\begin{array}{l}\text { Sulla spiaggia tra i fiumi Marta e Migone-Tarqui- } \\
\text { nia-Viterbo }\end{array}$ & $\begin{array}{l}42^{\circ} 12^{\circ} \mathrm{N} . \\
11^{\circ} 42^{\prime} \mathrm{E} .\end{array}$ & $5 \cdot X I I-1952$ & 07.00 & 30 & & A. Fioramini \\
\hline 6 & 937 & Spiaggia di Marina de Sisco-Capo Corso-Corsica & $\begin{array}{l}42^{\circ} 48^{\prime} \mathrm{N} . \\
\left(19^{\prime} 30^{\prime} \mathrm{E} \text {. }\right.\end{array}$ & $6-X I I-1952$ & & 31 & & Motroni \\
\hline 7 & 9.17 & Spiaggia del porto dell'Isola del Giglio-Grosseto & 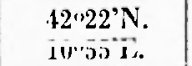 & $7-X I 1 \cdot 1952$ & 17.30 & 32 & & I. Piani \\
\hline 8 & 93.4 & $\begin{array}{l}\text { Spiaggia settentrionale dell'Isola del Giglio-Gros- } \\
\text { seto }\end{array}$ & $\begin{array}{l}12 " 23 \mathrm{~N} \\
10^{\circ} 51^{\circ} \mathrm{E} .\end{array}$ & $7-X 11-1952$ & & 32 & & R. Anichini \\
\hline 9 & 929 & ". & $m$ & ) & & 32 & & 》 \\
\hline 10 & 930 & Cala Scimo-S. Teresa di Gallura-Sassari & $\begin{array}{l}41^{\circ} 15^{\prime} \mathrm{N} . \\
09^{\circ} 12^{\prime} \mathrm{E}\end{array}$ & 9.XII.1952 & 09.00 & 34 & $\begin{array}{l}\text { Con vento di Gre- } \\
\text { co-Levante. }\end{array}$ & Q. Mannoni \\
\hline 11 & 921 & Cala Brandineri-Porto Cervo-La Maddalena-Sassari & $\begin{array}{l}41^{\circ} 07^{\prime} \mathrm{N} \\
09^{\circ 33} \mathrm{E} \text {. }\end{array}$ & $9 \cdot X I I \cdot 1952$ & 11.00 & 31 & & G. M. Orecchioni \\
\hline 12 & 919 & Sulla spiagata tra Tirrenia e Calambrone-Pisa & $\begin{array}{l}13^{\prime \prime} 37^{\prime} \mathrm{N} \\
10^{n} 17^{\prime} \mathrm{E} .\end{array}$ & $18 \cdot X I I-1952$ & 15.00 & +3 & & G. Calcagno \\
\hline 13 & 943 & $\begin{array}{l}\text { A ton m. circal dal lido di Lacco Ameno-Isolal } \\
\text { d'Ischia-Nipoli }\end{array}$ & $\begin{array}{l}40^{\circ} 45^{\prime} \mathrm{N} \\
13^{\prime \prime} 53^{\prime} \mathrm{E} .\end{array}$ & $9-1-1953$ & 12.00 & 65 & & M. Schiano \\
\hline
\end{tabular}


Quarto lancio sulla rolla Livorno-Bastia effettuato dalla M ve "Olbia " in if $43^{\circ} 04^{\prime} 00^{\prime \prime}$ N., $\%$. 09" $56^{\prime} 30^{\prime \prime}$ E.G., il 2 novembre 1952, alle ore 03.20 .

\section{RITROVAM ENT I}

\begin{tabular}{|c|c|c|c|c|c|c|c|c|}
\hline 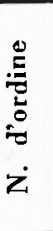 & 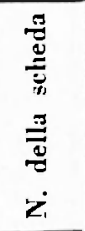 & $\mathrm{I}, C \wedge \mathrm{L}, \mathrm{I} \mathrm{T} \lambda$ & $\begin{array}{l}\text { Posizione } \\
\text { geografiea } \\
\text { approssimatal }\end{array}$ & Giorno & Ora & 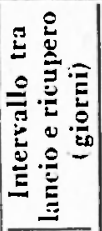 & Annotazioni & $\begin{array}{c}\text { Cognome di chi } \\
\text { ritrovo } \\
\text { il galleggiante }\end{array}$ \\
\hline 1 & 1008 & Spriaggia di S. Vincenzo-Livorno & $\begin{array}{l}43^{\circ} \cdot 06 \mathrm{~N} \\
10^{\circ} \cdot 32^{\circ} \mathrm{E} .\end{array}$ & $23-X 1-1952$ & 10.00 & 21 & & C. Cirenei \\
\hline 2 & 1007 & Spliaggia di S. Vincenzo-Livorno & ) & $n$ & 13.00 & 21 & & R. Formisano \\
\hline 3 & 1035 & Spiaggia di Torre del Iago Pucrini-Iueca & $\begin{array}{l}43^{\prime \prime} 19^{\circ} \mathrm{N} \\
10^{\prime \prime 16} \mathrm{E} .\end{array}$ & $9 . X 11-1952 ?$ & & 37 & $\begin{array}{l}\text { Pervenuta all'Istitu- } \\
\text { to Idrografico il } 10 \text {. }\end{array}$ & A. Fambrini \\
\hline 4 & 1003 & $\begin{array}{l}\text { A } 2 \text { miglia rirea dal porto di Centuri-Capo Corso- } \\
\text { Corsica }\end{array}$ & $\begin{array}{l}42 \cdot 58^{*} \mathrm{~N} \\
09 \cdot 18^{*} \mathrm{E} .\end{array}$ & $20-X 11-1952$ & & 48 & $\begin{array}{l}\text { XI1.1952 } \\
\text { Vento e corrente }\end{array}$ & A. Meliot \\
\hline 5 & 1009 & $\begin{array}{l}\text { A } 10 \text { m. circa dalla spiaggia Lo Mormorato-S. Te } \\
\text { resal di Gallura-Sassari }\end{array}$ & $\begin{array}{l}41^{\circ} 15^{\circ} \mathrm{N} . \\
09^{\prime \prime} 10^{\circ} \mathrm{E} .\end{array}$ & $22-X 11-1952$ & 11.00 & 50 & dit $\mathbf{N}$ & M. Fioredda \\
\hline 6 & 1036 & Costa NW. dell'Isola di Mal di Ventre.Cagliari & $\begin{array}{l}39059 \mathrm{~N} . \\
08 \cdot 18 \mathrm{E} .\end{array}$ & $9-11-1053$ & 09.00 & 99 & Vento di Maestrale & A. Ciubarda \\
\hline 7 & 1050 & Iido di Alghero-Sisssari & $\begin{array}{l}10^{\circ} 33^{\prime} \mathrm{N} \\
08^{\prime} 19^{\prime} \mathrm{E} .\end{array}$ & $11 \cdot 11.1953$ & 10.00 & 101 & & G. Geruggi \\
\hline 8 & 1039 & $\begin{array}{l}\text { Tra Punta dEecióa e Punta Manna Molino-Aiaco } \\
\text { rio-Corsion }\end{array}$ & $\begin{array}{l}10^{\circ} 36^{\circ} \mathrm{N} \\
08^{\circ}+5^{\prime} \mathrm{E}\end{array}$ & $16.11 \cdot 1953$ & 15.00 & 106 & Vento da SW. & P. Simonpietri \\
\hline 9 & 1031 & $\begin{array}{l}\text { Foce del fiume Platani-Bnrgo Bonsignori-Agri- } \\
\text { gento }\end{array}$ & $\begin{array}{l}37^{\circ} 24^{\prime} \mathrm{N} \\
13^{\circ} 16^{\circ} \mathrm{E} .\end{array}$ & $16.11 \cdot 1953$ & 16.30 & 106 & & D. Ferrigno \\
\hline 10 & 1002 & $\begin{array}{l}\text { Spiaggia di Isola Grande (Altavillia)-Marsala-Tra- } \\
\text { pani }\end{array}$ & $\begin{array}{l}37.53^{\prime} \mathrm{N} \\
12026^{\prime} \mathrm{E}\end{array}$ & $15 \cdot I V \cdot 1953$ & 10.30 & 161 & & A. Ciostil \\
\hline 11 & 1032 & Spiaggia di S. Iorenzo al Mare-Imperia & $\begin{array}{l}43055^{\prime} \mathrm{N} \\
08^{\circ} 06^{\prime} \mathrm{E}\end{array}$ & $21-V \cdot 1953$ & 11.00 & 2013 & & B. Bambini \\
\hline
\end{tabular}


Quarto lancio sulla rotta Genova-Porto Torres effettuato dalla M/ve "Civitavecchia " in $p \cdot 13^{\circ} 43^{\prime} 00^{\prime \prime} \mathrm{N}^{\prime}, \lambda 09^{\circ} 07^{\prime} 00^{\prime \prime}$ E.G., il 4 novembre 1952.

R I T R O V A M E N T I

\begin{tabular}{|c|c|c|}
\hline 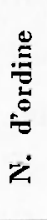 & 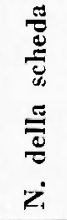 & L O O C A $A$ L I T \\
\hline 1 & 1091 & Spiaggia di Levanto-La Spezia \\
\hline 2 & 1089 & La Plaia Mannu-S. Vero Milis.Cagliari \\
\hline 3 & 1066 & $\begin{array}{l}\text { Spiaggia di Pevani-Colfo di Sagone-Aiarcio } \\
\text { Corsica }\end{array}$ \\
\hline 4 & 1097 & Spiaggia di Arenelli-Bastia-Corsica \\
\hline 5 & 1068 & $S_{\text {piaggial di Montroig-Tarragona-Spagna }}$ \\
\hline
\end{tabular}

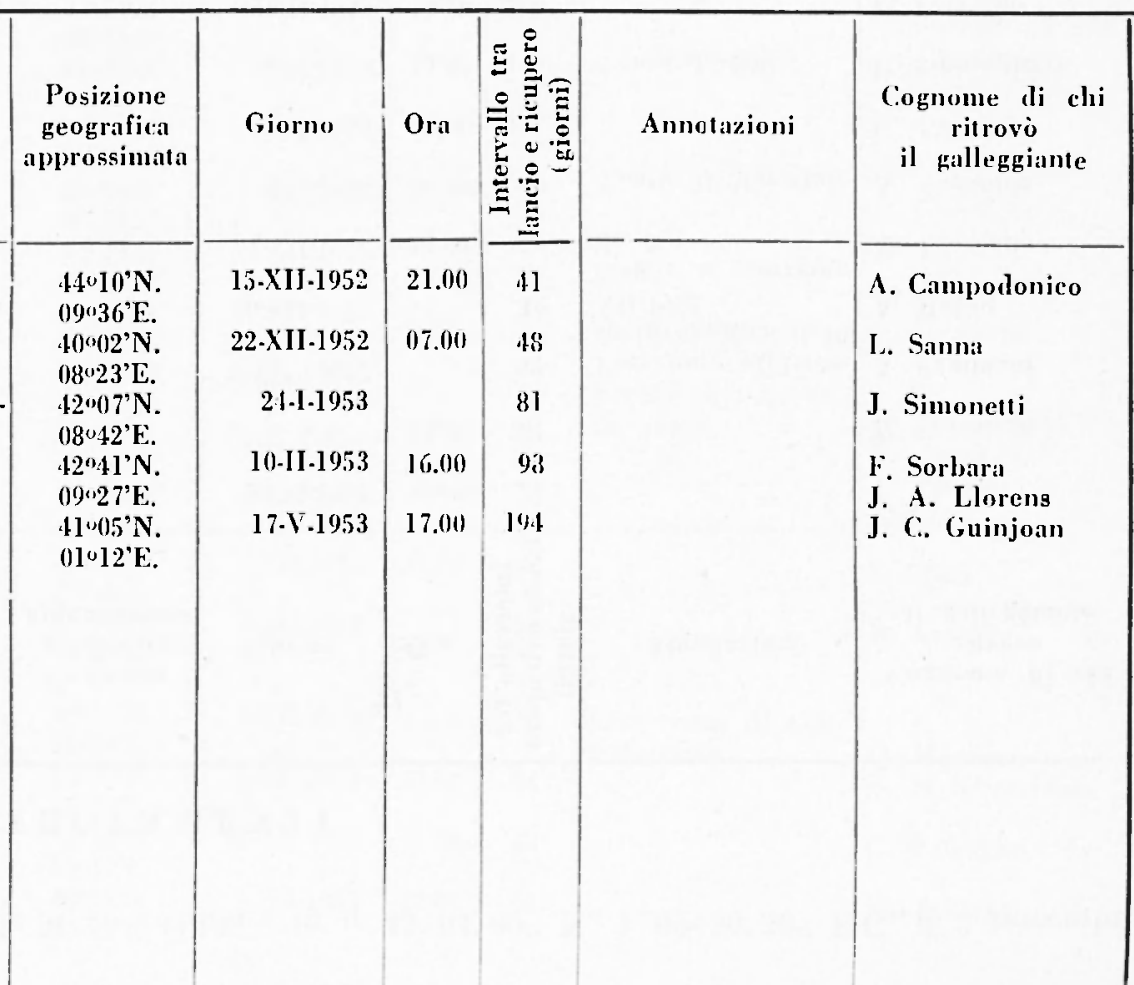

